Pure and Applied Mathematics Quarterly

Volume 7, Number 3

(Special Issue: In honor of

Jacques Tits)

$469-538,2011$

\title{
The Essentially Tame Jacquet-Langlands Correspondence for Inner Forms of GL(n)
}

\author{
Colin J. Bushnell and Guy Henniart \\ To Jacques Tits, on his eightieth birthday
}

\begin{abstract}
Let $F$ be a non-Archimedean local field, let $n \geqslant 1$ be an integer and $G=\mathrm{GL}_{n}(F)$. Let $G^{\prime}$ be an inner form of $G$, so that $G^{\prime}$ is isomorphic to $\mathrm{GL}_{m}(D)$, for a central $F$-division algebra of dimension $d^{2}, m d=n$. Using the structure theory of Sécherre and Stevens, we define a concept of parametric degree for irreducible cuspidal representations of $G^{\prime}$. We show that the image, under the Jacquet-Langlands correspondence, of the set of equivalence classes of irreducible cuspidal representations of $G$ is the set of equivalence classes of irreducible cuspidal representations of $G^{\prime}$ of parametric degree $n$. In earlier papers, we defined a notion of essential tameness for irreducible cuspidal representations of $G$. We generalize this to representations of $G^{\prime}$, and show that it is preserved by the Jacquet-Langlands correspondence. As in the earlier papers, the irreducible, essentially tame, cuspidal representations of $G$ admit an explicit parametrization in terms of admissible pairs, which can be explicitly related to the local Langlands correspondence. Here, we generalize this construction to the case of irreducible, essentially tame, cuspidal representations of $G^{\prime}$ of parametric degree $n$. We determine completely the behaviour of the two parametrizations relative to the Jacquet-Langlands correspondence. As a consequence, we prove a conjecture of Bushnell and Fröhlich of 1983.

Keywords: Explicit local Jacquet-Langlands correspondence, admissible pair, inner form, essentially tame representation, parametric degree, character formula
\end{abstract}

Received November 26, 2008.

Mathematics Subject Classification (2000). 22E50

Much of the work in this programme was carried out while the first-named author was visiting, and partly supported by, l'Université de Paris-Sud. Both authors were also partially supported by EU network "Arithmetical Algebraic Geometry". 
Let $F$ be a non-Archimedean local field with finite residue field of characteristic $p$. In a series of papers [11], [12], [14], we gave an explicit description of the local Langlands correspondence in the essentially tame case. In this paper, we give a parallel description of the Jacquet-Langlands correspondence. The results illuminate those of the earlier papers. They finally answer the most interesting of the questions left open in [6].

We emphasize that, in this paper, the characteristic of $F$ is arbitrary. The technical issues which forced us to restrict, in [14] in particular, to the characteristic zero case do not intervene here. The parts of [14] to which we appeal in this paper are valid in all characteristics.

1. Let $n \geqslant 1$ be an integer and let $G$ be an inner form of $\mathrm{GL}_{n}(F)$. Thus there is a central $F$-division algebra $D$, of dimension $d^{2}, d \geqslant 1$, such that $G \cong \mathrm{GL}_{m}(D)$, $m d=n$. Let $\mathcal{A}_{m}^{\square}(D)$ denote the set of equivalence classes of irreducible smooth representations of $G$ which are essentially square-integrable, modulo the centre $F^{\times}$of $G$. Thus, in particular, $\mathcal{A}_{n}^{\square}(F)$ denotes the set of equivalence classes of irreducible smooth representations of $\mathrm{GL}_{n}(F)$ which are essentially squareintegrable modulo centre.

The Jacquet-Langlands correspondence gives a canonical bijection

$$
\boldsymbol{j}: \mathcal{A}_{n}^{\square}(F) \stackrel{\approx}{\longrightarrow} \mathcal{A}_{m}^{\square}(D),
$$

specified by a character relation on elliptic regular elements.

The idea of the correspondence, and the basic method for proving it, both originate with Jacquet, Langlands [27] which treats the case $n=2$ when $F$ has characteristic zero. For the general case with $F$ of characteristic zero, see Deligne, Kazhdan, Vignéras [21], also Rogawski [37]. The positive characteristic case is treated by Badulescu [2], with a supplement in [16] required for this paper.

2. The set $\mathcal{A}_{m}^{\square}(D)$ contains the set $\mathcal{A}_{m}^{0}(D)$ of equivalence classes of irreducible cuspidal representations of $\mathrm{GL}_{m}(D)$. An early step in this paper identifies the image of $\mathcal{A}_{n}^{0}(F)$ in $\mathcal{A}_{m}^{\square}(D)$ under the map (1.1).

This relies on a notion of parametric degree for representations $\pi \in \mathcal{A}_{m}^{\square}(D)$, generalizing the cases of $\mathcal{A}_{n}^{\square}(F), \mathcal{A}_{1}^{\square}(D)$ treated in [9]. The definition in [9] uses the classification theory for cuspidal representations of $\mathrm{GL}_{n}(F)$ in [17] and the analogous results of Broussous [4] or Zink [43] for $\mathrm{GL}_{1}(D)$. The necessary 
elements of the classification are now available for $\mathrm{GL}_{m}(D)$ in the paper [41] of Sécherre and Stevens, the culmination of Sécherre's programme [38], [39], [40].

For $\pi \in \mathcal{A}_{m}^{\square}(D)$, the parametric degree $\delta(\pi)$ is a positive integer dividing $n$. If $\delta(\pi)=n$, then $\pi$ is cuspidal. The converse holds for $\mathrm{GL}_{n}(F)$ but not in general. Just as in [9], the parametric degree is determined by the normalized formal degree. Since the Jacquet-Langlands correspondence preserves the normalized formal degree [21], [37], [16], it must also preserve the parametric degree. Thus

$$
\boldsymbol{j}\left(\mathcal{A}_{n}^{0}(F)\right)=\left\{\pi \in \mathcal{A}_{m}^{\square}(D): \delta(\pi)=n\right\} \subset \mathcal{A}_{m}^{0}(D) .
$$

For $\pi \in \mathcal{A}_{m}^{\square}(D)$, let $t(\pi)$ denote the number of unramified characters $\chi$ of $F^{\times}$ such that $\chi \pi \cong \pi$. (Here we use the customary notation: $\chi \pi$ is the representation $g \mapsto \chi(\operatorname{Nrd} g) \pi(g)$, where Nrd denotes the reduced norm map $\operatorname{GL}_{m}(D) \rightarrow F^{\times}$.) The integer $t(\pi)$ divides $\delta(\pi)$. One says that $\pi$ is essentially tame if $p$ does not divide $\delta(\pi) / t(\pi)$. In particular, if $p$ does not divide $n$, every $\pi \in \mathcal{A}_{m}^{\square}(D)$ is essentially tame.

We denote by $\mathcal{A}_{m}^{\text {et }}(D)$ the set of classes of representations $\pi \in \mathcal{A}_{m}^{\square}(D)$ which have $\delta(\pi)=n$ and are essentially tame. This generalizes the definition of $\mathcal{A}_{n}^{\text {et }}(F)$ in [11].

Because it preserves both the parametric degree and the $t$-invariant, the Jacquet-Langlands correspondence (1.1) induces a canonical bijection

$$
j: \mathcal{A}_{n}^{\mathrm{et}}(F) \stackrel{\approx}{\longrightarrow} \mathcal{A}_{m}^{\mathrm{et}}(D)
$$

The aim of the paper is to make this map explicit, in a manner parallel to that of [11], [12], [14].

3. Such an aim, of course, requires an explicit description of both sets $\mathcal{A}_{n}^{\text {et }}(F)$, $\mathcal{A}_{m}^{\text {et }}(D)$. For the first of them, we have the "naïve correspondence" of [11]. This is a canonical bijection

$$
\begin{aligned}
P_{n}(F) & \longrightarrow \mathcal{A}_{n}^{\mathrm{et}}(F), \\
(E / F, \xi) & \longmapsto{ }_{F} \Pi_{\xi},
\end{aligned}
$$

where $P_{n}(F)$ is the set of $F$-isomorphism classes of admissible pairs $(E / F, \xi)$ of degree $n$ (the definition is recalled in 4.1 below). The map (3.1) generalizes 
the constructions of Howe's early paper [26]. Our first task is to produce an analogous bijection

$$
\begin{aligned}
P_{n}(F) & \longrightarrow \mathcal{A}_{m}^{\mathrm{et}}(D), \\
(E / F, \xi) & \longmapsto{ }_{D} \Pi_{\xi} .
\end{aligned}
$$

4. Here, and indeed throughout the paper, we rely on [38-41]. These papers contain all the necessary elements of a complete theory of types [19] for the groups $\mathrm{GL}_{m}(D)$, directly generalizing [17] and [4]. In particular, the cuspidal representations of $\mathrm{GL}_{m}(D)$ are explicitly presented as induced representations. The description extends in essence to all of $\mathcal{A}_{m}^{\square}(D)$. However, the theory exhibits some novel technical difficulties, as compared with the "split" case of $\mathrm{GL}_{n}(F)$, and it has not yet reached the same level of completeness. Consequently, we cannot simply follow [11] to construct the bijection (3.2): our approach must be more indirect.

We start in $\S \S 3,4$ by attaching to $\pi \in \mathcal{A}_{m}^{\text {et }}(D)$ an admissible pair, using the classification in [41]. The process involves many choices, and there is no guarantee, at first, that it has any intrinsic meaning. (However, in the case of $\mathrm{GL}_{1}(D)$ with $p$ not dividing $\operatorname{dim}_{F} D$, it is easy to see that it gives the bijection of [20], [28], on which [6] is based.)

Let $\pi \in \mathcal{A}_{m}^{\text {et }}(D)$ be totally ramified, in the sense that $t(\pi)=1$. An admissible pair $(E / F, \xi)$ attached to $\pi$ then has $E / F$ totally ramified. Some simple calculations show that $(E / F, \xi)$ can be recovered from the values of the character function $\operatorname{tr} \pi$ of $\pi$ at certain elliptic regular elements. The pair is therefore uniquely determined by $\pi$, up to $F$-isomorphism. Further calculation shows that $\pi$ is matched, via the Jacquet-Langlands correspondence (2.2), with the representation ${ }_{F} \Pi_{\nu \xi} \in \mathcal{A}_{n}^{\text {et }}(F)$, where $\nu$ is an unramified quadratic character of $F^{\times}$ depending only on the integers $m, d$. This step is to be found in $\S 5$, the formula for $\nu$ being given in 5.3 .

5. The core of the paper is $\S 6$. We take a general representation $\pi \in \mathcal{A}_{m}^{\text {et }}(D)$, with an attached admissible pair $(E / F, \xi)$ (possibly one of many). We calculate the character value $\operatorname{tr} \pi(\zeta u)$ at suitable elements $\zeta u$ near a root of unity $\zeta \in$ $E$, that generates the maximal unramified sub-extension $K / F$ of $E / F$. The outcome is an expression for $\operatorname{tr} \pi(\zeta u)$ in terms of $(E / F, \xi)$ and character values 
$\operatorname{tr} \rho(u)$, for various essentially tame, totally ramified, cuspidal representations $\rho$ of the $G$-centralizer of $K$. We know, from $\S 5$, how such representations relate to representations of split groups and to admissible pairs. We are able to deduce that the process, of attaching an admissible pair to $\pi \in \mathcal{A}_{m}^{\text {et }}(D)$, yields the inverse of a canonical bijection (3.2). This is the Parametrization Theorem of 6.1.

We further show that, if $(E / F, \xi) \in P_{n}(F)$, then ${ }_{D} \Pi_{\xi}$ is of the form $\boldsymbol{j}\left({ }_{F} \Pi_{\nu \xi}\right)$, for a canonically determined, tame, quadratic character $\nu$ of $E^{\times}$: this is the First Comparison Theorem 6.1.

We calculate the character ${ }_{D} \nu_{\xi}=\nu_{\xi}$ in terms of the "symplectic signs" ubiquitous in [14]. We interpret these signs in terms of $m, D$ and $(E / F, \xi)$ to give an explicit formula for the restriction ${ }_{D} \nu_{\xi} \mid U_{E}$ : see 6.9 Corollary and (6.7.4).

It remains only to calculate the value of the tamely ramified character ${ }_{D} \nu_{\xi}$ at one prime element of $E$. This is the subject of the Second Comparison Theorem of 7.1. We leave the answer in terms of symplectic signs: a calculation along the lines of the proof of 8.4 Theorem of [14] is necessary to reduce it to numerical form.

Together, the two Comparison Theorems show:

Theorem A. Let $D$ be a central $F$-division algebra of dimension $d^{2}, d \geqslant 1$, let $m \geqslant 1$ be an integer and set $n=m d$. If $(E / F, \xi)$ is an admissible pair with $[E: F]=n$, there is a canonically determined, tamely ramified character $\nu={ }_{D} \nu_{\xi}$ such that $(E / F, \nu \xi)$ is admissible, $\nu^{2}=1$, and

$$
{ }_{D} \Pi_{\xi}=\boldsymbol{j}\left({ }_{F} \Pi_{\nu \xi}\right),
$$

where $\boldsymbol{j}: \mathcal{A}_{n}^{\mathrm{et}}(F) \rightarrow \mathcal{A}_{m}^{\mathrm{et}}(D)$ is the Jacquet-Langlands correspondence.

The determination of ${ }_{D} \nu_{\xi}$ in the theorems of 6.1, 7.1 implies immediately:

Theorem B. For $i=1,2$, let $\left(E / F, \xi_{i}\right)$ be an admissible pair with $[E: F]=n$. If $\xi_{1}^{-1} \xi_{2}$ is tamely ramified, then ${ }_{D} \nu_{\xi_{1}}={ }_{D} \nu_{\xi_{2}}$.

A closer examination of the form of the character ${ }_{D} \nu_{\xi}$, which we omit here, yields:

Theorem C. For $i=1,2$, let $D_{i}$ be a central $F$-division algebra and suppose that $\operatorname{dim}_{F} D_{1}=\operatorname{dim}_{F} D_{2}=d^{2}, d \geqslant 1$. If $(E / F, \xi)$ is an admissible pair with $[E: F]$ divisible by $d$, then ${ }_{D_{1}} \nu_{\xi}={ }_{D_{2}} \nu_{\xi}$. 
6. We put these results in the context of the Langlands correspondence: since we now appeal to essential features of [14], we have to assume that $F$ has characteristic zero. Let $\mathcal{W}_{F}$ denote the Weil group of $F$, and let $\mathcal{G}_{n}^{0}(F)$ denote the set of equivalence classes of irreducible smooth representations of $\mathcal{W}_{F}$ of dimension $n$. If $(E / F, \xi)$ is an admissible pair with $[E: F]=n$, we may view $\xi$ as a character of $\mathcal{W}_{E}$ via class field theory and form the induced representation

$$
{ }_{F} \Sigma_{\xi}=\operatorname{Ind}_{\mathcal{W}_{E}}^{\mathcal{W}_{F} \xi}
$$

of $\mathcal{W}_{F}$. This representation is irreducible, and we so obtain a bijection

$$
(E / F, \xi) \longmapsto{ }_{F} \Sigma_{\xi}
$$

of $P_{n}(F)$ with the subset $\mathcal{G}_{n}^{\text {et }}(F)$ of classes of representations $\sigma \in \mathcal{G}_{n}^{0}(F)$ which are essentially tame, in an obvious sense [11]. The Langlands correspondence then induces a canonical bijection

$$
{ }_{F} \mathcal{L}: \mathcal{G}_{n}^{\mathrm{et}}(F) \longrightarrow \mathcal{A}_{n}^{\mathrm{et}}(F) \text {. }
$$

This is of the form ${ }_{F} \Sigma_{\xi} \mapsto{ }_{F} \Pi_{\mu \xi}$, for a canonically determined, tamely ramified character $\mu={ }_{F} \mu_{\xi}$ of $E^{\times}$.

Composing with the Jacquet-Langlands correspondence

$$
j: \mathcal{A}_{n}^{\mathrm{et}}(F) \longrightarrow \mathcal{A}_{m}^{\mathrm{et}}(D),
$$

we obtain the Langlands correspondence

$$
{ }_{D} \mathcal{L}: \mathcal{G}_{n}^{\mathrm{et}}(F) \longrightarrow \mathcal{A}_{m}^{\mathrm{et}}(D) \text {. }
$$

This is bijective. Combining the results of this paper with those of [12], [14], we get:

\section{Theorem D.}

(1) Let $(E / F, \xi)$ be an admissible pair in which $[E: F]=n$. There is a canonically determined, tamely ramified character $\lambda={ }_{D} \lambda_{\xi}$ of $E^{\times}$such that

$$
{ }_{D} \mathcal{L}:{ }_{F} \Sigma_{\xi} \longmapsto{ }_{D} \Pi_{\lambda \xi} .
$$


Indeed, ${ }_{D} \lambda_{\xi}={ }_{F} \mu_{\xi} \cdot{ }_{D} \nu_{\xi}$.

(2) If $\left(E / F, \xi_{i}\right), i=1,2$, are admissible pairs such that $\xi_{1}^{-1} \xi_{2}$ is tamely ramified, then

$$
{ }_{D} \lambda_{\xi_{1}}={ }_{D} \lambda_{\xi_{2}}
$$

(3) The character $\lambda={ }_{D} \lambda_{\xi}$ satisfies $\lambda^{4}=1$ and $\lambda^{2} \mid U_{E}=1$.

This result was conjectured, for the case $m=1$, in [6]. However, the values for $\lambda$ proposed there (and in [34]) are no closer to reality than those proposed by Moy [33] for the character $F \mu_{\xi}$ : see the discussion in [12] 2.2.

7. A number of discrete technical issues, of possibly wider interest, arise in the course of the paper. The first of these, already mentioned, is the relation between the (algebraic) parametric degree and the (analytic) formal degree. This is general in nature, independent of any consideration of characteristic and of our underlying tameness hypotheses. We have therefore given it separate treatment in Appendix 1.

At a critical point in the character calculations of $\S 7$, we have to evaluate the character of a "wide extension" (aka " $\beta$-extension") of the Heisenberg representation containing a simple character. The same problem arose at the corresponding point of [14], where we returned to the original definition in [17]. The extra complexity of the general case in [39] makes that a daunting prospect here. We have substituted a general argument, based on the interaction between the Glauberman correspondence and intertwining of representations. This simplifies a parallel result of Stevens (the Principal Lemma 2.4 of [42]), so we have included a proof in Appendix 2.

\section{Notation}

Throughout, $F$ denotes a non-Archimedean local field. The discrete valuation ring in $F$ is denoted $\mathfrak{o}_{F}$, and the maximal ideal of $\mathfrak{o}_{F}$ is $\mathfrak{p}_{F}$. We write $\mathbb{k}_{F}=$ $\mathfrak{o}_{F} / \mathfrak{p}_{F}, q=q_{F}=\left|\mathbb{k}_{F}\right|$, and we let $p$ denote the characteristic of $\mathbb{k}_{F}$. We set $U_{F}=U_{F}^{0}=\mathfrak{o}_{F}^{\times}$, and $U_{F}^{k}=1+\mathfrak{p}_{F}^{k}, k \geqslant 1$. We write $v_{F}$ for the canonical (surjective) valuation map $F^{\times} \rightarrow \mathbb{Z}$. We denote by $\boldsymbol{\mu}_{F}$ the group of roots of unity in $F$ of order relatively prime to $p$. 
We use the obvious analogues of these conventions for a finite field extension $E / F$. Additionally, we denote by $\mathrm{N}_{E / F}$ and $\operatorname{Tr}_{E / F}$ the relative norm and trace maps $E \rightarrow F$ respectively.

If $D$ is a central $F$-division algebra of finite dimension, then $\mathfrak{o}_{D}$ is the discrete valuation ring in $D$ and $\mathfrak{p}_{D}$ is the unique maximal ideal of $\mathfrak{o}_{D}$. We denote by $\mathbb{k}_{D}$ the field $\mathfrak{o}_{D} / \mathfrak{p}_{D}$ : this is finite, with $q_{D}=q_{F}^{d}$ elements, where $d^{2}=\operatorname{dim}_{F} D$. We set $U_{D}=\mathfrak{o}_{D}^{\times}$and $U_{D}^{k}=1+\mathfrak{p}_{D}^{k}, k \geqslant 1$.

If $A$ is a finite-dimensional, central simple $F$-algebra, then $\operatorname{Nrd}_{A}: A^{\times} \rightarrow F^{\times}$ is the reduced norm map.

Most notation concerning the internal structure of representations is introduced as it arises, but is kept closely parallel to the standard usage of [17].

\section{Classical character relations}

We fix an integer $n \geqslant 1$. We are concerned with locally profinite groups of the form $G=\mathrm{GL}_{m}(D)$, where $D$ is a central $F$-division algebra of finite dimension $d^{2}, d \geqslant 1$, and $n=m d$. We set $A=\mathrm{M}_{m}(D)$, so that $G=A^{\times}$.

To reach the effective starting point of the paper, we have to gather threads from several sources and weave them together. In this section, we concentrate on ideas from harmonic analysis, some elementary, some deriving ultimately from the trace formula. The main point is to recall the Jacquet-Langlands correspondence and its main properties. In characteristic zero, we can largely rely on the original sources [21], [37]. In positive characteristic, the situation is more involved. The principal result is given in [2], relying on [1], [29], [30]. A crucial point for us comes from the recent [16].

One proof has been deferred to the end of the next section, as it will be easier to treat with the machinery of hereditary orders to hand.

1.1. We recall some basic results from the Appendix to [8]. Those pages were written for the split case $G=\mathrm{GL}_{n}(F)$, but no attention was paid to the characteristic of $F$. The proofs apply unchanged to the present situation.

Let $g \in G$ and denote by $f_{g}^{A}(t) \in F[t]$ the reduced characteristic polynomial of $g$. For us, $g$ is regular if $f_{g}^{A}(t)$ has no repeated root in an algebraic closure of $F$. It is elliptic regular if it is regular and $f_{g}^{A}(t)$ is irreducible over $F$. We denote by $G_{\mathrm{reg}}, G_{\mathrm{reg}}^{\mathrm{ell}}$ the sets of regular or elliptic regular elements of $G$ respectively. 
Remark. In [8], we introduced the notion of a quasi-regular element of $G$ : an element $g \in G$ is quasi-regular if $f_{g}^{A}(t)$ has no repeated irreducible factor over $F$. An element is elliptic quasi-regular if it is quasi-regular and $f_{g}^{A}(t)$ is irreducible over F. Everything we do applies equally, on replacing "regular" by "quasiregular" and "elliptic regular" by "elliptic quasi-regular".

We fix a Haar measure $\mu_{G}$ on $G$ and use it to define the structure of a convolution algebra on the space $\mathcal{H}(G)$ of locally constant, compactly supported functions $G \rightarrow \mathbb{C}$. If $(\pi, V)$ is an irreducible smooth representation of $G$, we extend $\pi$ to an algebra homomorphism $\pi: \mathcal{H}(G) \rightarrow \operatorname{End}_{\mathbb{C}}(V)$ in the standard way [13] $\S 4$. Since $(\pi, V)$ is admissible, each operator $\pi(\varphi), \varphi \in \mathcal{H}(G)$, has finite-dimensional range and so its $\operatorname{trace} \operatorname{tr} \pi(\varphi)$ is defined. As in [8] A.11, we have:

Proposition. There is a locally constant function $\operatorname{tr} \pi: G_{\mathrm{reg}} \rightarrow \mathbb{C}$ such that

$$
\operatorname{tr} \pi(\varphi)=\int_{G} \operatorname{tr} \pi(g) \varphi(g) d \mu_{G}(g),
$$

for all $\varphi \in \mathcal{H}(G)$ with support contained in $G_{\text {reg }}$.

One refers to the function $\operatorname{tr} \pi$ as the character of $\pi$.

1.2. We need more detail in one particular case. We take an irreducible cuspidal representation $\pi$ of $G$ and assume it is given as an induced representation

$$
\pi \cong c-\operatorname{Ind}_{J}^{G} \Lambda,
$$

where $\boldsymbol{J}$ is an open subgroup of $G$, compact modulo the centre $F^{\times}$of $G$, and $\Lambda$ is an irreducible smooth representation of $\boldsymbol{J}$. In particular, $\operatorname{dim} \Lambda$ is finite. If $K$ is a compact open subgroup of $G$, A.14 of [8] gives the expression

$$
\operatorname{tr} \pi(g)=\sum_{x \in K \backslash G / J} \sum_{y \in K x \boldsymbol{J} / \boldsymbol{J}} \operatorname{tr} \Lambda\left(y^{-1} g y\right), \quad g \in G_{\text {reg }} .
$$

Here, we regard $\operatorname{tr} \Lambda$ as a function on $G$, vanishing outside of $\boldsymbol{J}$. If we confine $g$ to a fixed compact subset $C$ of $G_{\text {reg }}$, only finitely many of the inner terms

$$
\sum_{y \in K x \boldsymbol{J} / \boldsymbol{J}} \operatorname{tr} \Lambda\left(y^{-1} g y\right)
$$

can be non-zero loc. cit.

This uniform convergence property of (1.2.1) is stronger than we need for the purely algebraic manipulations in this paper. The following non-uniform version is easier to use here. 
Lemma. Let $g \in G_{\mathrm{reg}}^{\mathrm{ell}}$. There exist only finitely many cosets $x \boldsymbol{J} \in G / \boldsymbol{J}$ such that $x^{-1} g x \in \boldsymbol{J}$. In particular, only finitely many terms $\operatorname{tr} \Lambda\left(x^{-1} g x\right), x \in G / \boldsymbol{J}$, are non-zero.

Proof. The $F$-subalgebra $F[g]$ of $A$ is a field, and any two $F$-embeddings of $F[g]$ in $A$ are $G$-conjugate, by the Skolem-Noether Theorem. It follows that the $G$-conjugacy class $C(g)=\left\{x^{-1} g x: x \in G\right\}$ of $g$ is the set of zeros of the polynomial function $h \mapsto f_{g}^{A}(h), h \in G$. That is, $C(g)$ is closed in $G$ and hence locally compact. Consider the intersection $\boldsymbol{J} \cap C(g)$. The reduced norm map $\operatorname{Nrd}_{A}$ is constant on $C(g)$. On the other hand, $\boldsymbol{J}$ has a unique maximal compact subgroup $J=\left\{x \in \boldsymbol{J}:\left\|\operatorname{Nrd}_{A} x\right\|=1\right\}$ and $\boldsymbol{J} / J$ is cyclic. It follows that $\boldsymbol{J} \cap C(g)$ is compact.

The $G$-centralizer of $g$ is the group $F[g]^{\times}$, and $F[g]^{\times} / F^{\times}$is compact. The set $S$ of $x \in G$ for which $x^{-1} g x \in \boldsymbol{J}$ satisfies $\boldsymbol{J} S F[g]^{\times}=S$. The local compactness of $C(g)$ implies, via Arens' Theorem [32] 2.13, that the canonical map $G / F[g]^{\times} \rightarrow$ $C(g)$ is a homeomorphism. Consequently, the image $S / F[g]^{\times}$of $S$ in $G / F[g]^{\times}$ is homeomorphic to $\boldsymbol{J} \cap C(g)$ and is compact. Hence $S$ is compact modulo $F^{\times}$. The quotient space $\boldsymbol{J} \backslash S$ is therefore finite, as required.

The lemma allows us to use the Mackey Formula (1.2.1) in the form

$$
\operatorname{tr} \pi(g)=\sum_{x \in G / \boldsymbol{J}} \operatorname{tr} \Lambda\left(x^{-1} g x\right), \quad g \in G_{\mathrm{reg}}^{\mathrm{ell}}
$$

without having to be concerned about convergence issues.

1.3. The group $G$ acts on the set $G_{\text {reg }}^{\text {ell }}$ by conjugation. The map $g \mapsto f_{g}^{A}(t)$ induces a bijection between $G \backslash G_{\text {reg }}^{\text {ell }}$ and the set of monic, irreducible, separable polynomials over $F$ of degree $n$ ( $c f .2 .1$ below).

The same applies if we replace $A$ by another $n^{2}$-dimensional, central simple $F$-algebra $A^{\prime}$ and set $G^{\prime}=A^{\prime \times}$. Thus we have a canonical bijection $G \backslash G_{\text {reg }}^{\text {ell }} \cong$ $G^{\prime} \backslash G_{\text {reg. }}^{\text {ell }}$. We say that elements $g \in G_{\text {reg }}^{\text {ell }}, g^{\prime} \in G_{\text {reg }}^{\text {ell }}$ are associate if their conjugacy classes correspond under this bijection, that is, if $f_{g^{\prime}}^{A^{\prime}}=f_{g}^{A}$.

If $g \in G_{\mathrm{reg}}^{\mathrm{ell}}$, it is in practice safe to use the same notation $g$ for an element of $G_{\text {reg }}^{\text {ell }}$ which is associate to $g$. 
1.4. Let $A=\mathrm{M}_{m}(D)$ and $G=A^{\times}=\mathrm{GL}_{m}(D)$. If $\pi$ is a smooth representation of $G$ and $\chi$ is a character of $F^{\times}$, we denote by $\chi \pi$ the representation

$$
\chi \pi: g \longmapsto \chi\left(\operatorname{Nrd}_{A} g\right) \pi(g), \quad g \in G .
$$

Let $\mathcal{A}_{m}^{\square}(D)$ denote the set of equivalence classes of irreducible smooth representations of $G$ which are essentially square-integrable modulo the centre $F^{\times}$of $G$.

Jacquet-Langlands Correspondence. Let $D, D^{\prime}$ be central F-division algebras of dimension $d^{2}, d^{\prime 2}$ respectively. Let $m, m^{\prime}$ be positive integers such that $m d=m^{\prime} d^{\prime}=n$. Set $G=\mathrm{GL}_{m}(D), G^{\prime}=\mathrm{GL}_{m^{\prime}}\left(D^{\prime}\right)$. There is a unique bijection

$$
\begin{aligned}
j: \mathcal{A}_{m}^{\square}(D) & \longrightarrow \mathcal{A}_{m^{\prime}}^{\square}\left(D^{\prime}\right), \\
\pi & \longmapsto \pi^{\prime},
\end{aligned}
$$

with the following property. If $g \in G_{\mathrm{reg}}^{\mathrm{ell}}$ and if $g^{\prime} \in G_{\mathrm{reg}}^{\text {ell }}$ is associate to $g$, then

$$
(-1)^{m} \operatorname{tr} \pi(g)=(-1)^{m^{\prime}} \operatorname{tr} \pi^{\prime}\left(g^{\prime}\right) .
$$

We refer to the map $j$ as the Jacquet-Langlands correspondence between $G$ and $G^{\prime}$.

We note some obvious properties, using the same notation.

(1.4.2) If $\pi \in \mathcal{A}_{m}^{\square}(D)$ and $\pi^{\prime}=\boldsymbol{j} \pi$, then the central characters $\omega_{\pi}, \omega_{\pi^{\prime}}$ of $\pi, \pi^{\prime}$ are equal.

(1.4.3) If $\pi \in \mathcal{A}_{m}^{\square}(D)$ and $\pi^{\prime}=\boldsymbol{j} \pi$, then $\boldsymbol{j}(\chi \pi)=\chi \pi^{\prime}$, for any character $\chi$ of $F^{\times}$.

For an irreducible smooth representation $\pi$ of (say) $G$, we let $t(\pi)$ denote the number of unramified characters $\chi$ of $F^{\times}$such that $\chi \pi \cong \pi$. From (1.4.3) we deduce that:

(1.4.4) If $\pi \in \mathcal{A}_{m}^{\square}(D)$ and $\pi^{\prime}=\boldsymbol{j} \pi$, then $t(\pi)=t\left(\pi^{\prime}\right)$.

We shall also need the following fact, which is bound up in the proof of the existence and uniqueness of the Jacquet-Langlands correspondence in all cases. We treat it as a consequence from which we shall derive stronger results of the same kind. 
(1.4.5) Let $\pi_{1}, \pi_{2}, \ldots, \pi_{r}$ be distinct elements of $\mathcal{A}_{m}^{\square}(D)$, and set $G=\mathrm{GL}_{m}(D)$. The set of functions on $G_{\mathrm{reg}}^{\mathrm{ell}}$, defined by

$$
\left\{\operatorname{tr} \pi_{i} \mid G_{\mathrm{reg}}^{\mathrm{ell}}: 1 \leqslant i \leqslant r\right\},
$$

is linearly independent.

Proof. Let $C$ be a central $F$-division algebra of dimension $n^{2}=m^{2} d^{2}$, and write $G^{\prime}=\mathrm{GL}_{1}(C)$. The set $G^{\prime}$ rell is dense in $G^{\prime}$ and, for $\pi \in \mathcal{A}_{1}^{\square}(C)$, the function $\operatorname{tr} \pi$ is locally constant on $G^{\prime}$. The result is therefore elementary for $G^{\prime}$ and holds in general as a consequence of (1.4.1).

1.5. Write $G=\mathrm{GL}_{m}(D)$ and let $(\pi, V) \in \mathcal{A}_{m}^{\square}(D)$ be square-integrable modulo centre. Let $(\check{\pi}, \check{V})$ be the contragredient of $(\pi, V)$ and let $\dot{\mu}_{G}$ be a Haar measure on $G / F^{\times}$. For $v_{i} \in V$ and $\check{v}_{i} \in \check{V}$, we have the first Schur orthogonality relation

$$
\int_{G / F^{\times}}\left\langle\check{\pi}(g) \check{v}_{1}, v_{1}\right\rangle\left\langle\check{v}_{2}, \pi(g) v_{2}\right\rangle d \dot{\mu}_{G}(g)=d\left(\pi, \dot{\mu}_{G}\right)^{-1}\left\langle\check{v}_{1}, v_{2}\right\rangle\left\langle\check{v}_{2}, v_{1}\right\rangle,
$$

where $d\left(\pi, \dot{\mu}_{G}\right)$ is a positive real constant called the formal degree of $\pi$ relative to $\dot{\mu}_{G}$. (See, for instance, [13] 10a.2 for an exposition of this topic.)

Clearly, if $\chi$ is a character of $F^{\times}$which is unitary, in that $|\chi|=1$, then $\chi \pi$ is square-integrable mod. centre and $d\left(\chi \pi, \dot{\mu}_{G}\right)=d\left(\pi, \dot{\mu}_{G}\right)$. For an arbitrary $\pi \in \mathcal{A}_{m}^{\square}(D)$, there is an unramified character $\chi$ of $F^{\times}$such that $\pi_{0}=\chi \pi$ is square-integrable mod. centre. We define $d\left(\pi, \dot{\mu}_{G}\right)=d\left(\pi_{0}, \dot{\mu}_{G}\right)$.

We discuss ways in which the Haar measure $\dot{\mu}_{G}$ may be conveniently normalized. In the case $m=1$, the group $D^{\times} / F^{\times}$is compact, so one may choose $\dot{\mu}_{D^{\times}}$ to give $D^{\times} / F^{\times}$mass 1 . For this choice of Haar measure, every $\pi \in \mathcal{A}_{1}^{\square}(D)$ has formal degree equal to $\operatorname{dim} \pi$.

In general, let $\pi \in \mathcal{A}_{m}^{\square}(D)$. The cuspidal support of $\pi$ then consists of unramified twists of a cuspidal representation $\sigma \in \mathcal{A}_{m^{\prime}}^{\square}(D)$, for some divisor $m^{\prime}$ of $m$. In the case where $m^{\prime}=1$ and $\sigma$ is of dimension 1 , the representation $\pi$ is of the form $\chi \cdot \mathrm{St}_{G}$, where $\chi$ is a unitary character of $F^{\times}$and $\mathrm{St}_{G}$ is the Steinberg representation of $G$. (We recall more of this matter in Appendix 1 below.)

There is a unique Haar measure $\ddot{\mu}_{G}$ on $G / F^{\times}$such that

$$
d\left(\mathrm{St}_{G}, \ddot{\mu}_{G}\right)=1 .
$$


We set

$$
\operatorname{deg} \pi=d\left(\pi, \ddot{\mu}_{G}\right), \quad \pi \in \mathcal{A}_{m}^{\square}(D),
$$

and call $\operatorname{deg} \pi$ the normalized formal degree of $\pi$.

The following result may be found in [21] when $F$ has characteristic zero, in [16] when $F$ has characteristic $p$ (but the scheme of proof in [16] applies equally in characteristic zero).

\section{Theorem.}

(1) Let $\pi \in \mathcal{A}_{m}^{\square}(D)$; there is an open neighbourhood $\mathcal{N}$ of 1 in $G$ such that

$$
\operatorname{tr} \pi(g)=(-1)^{m-1} \operatorname{deg} \pi, \quad g \in \mathcal{N} \cap G_{\text {reg }}^{\text {ell }}
$$

(2) Using the notation of (1.4), we have

$$
\begin{gathered}
\operatorname{deg} \pi=\operatorname{deg} \pi^{\prime}, \\
\text { for every } \pi \in \mathcal{A}_{m}^{\square}(D) \text { and } \pi^{\prime}=\boldsymbol{j} \pi \in \mathcal{A}_{m^{\prime}}^{\square}\left(D^{\prime}\right) .
\end{gathered}
$$

1.6. If $G$ is any of the groups $\mathrm{GL}_{m}(D)$, an element $g \in G$ is called pro-unipotent if $g^{p^{r}} \rightarrow 1$ as $r \rightarrow \infty$. Let ${ }_{\mathrm{u}} G_{\mathrm{reg}}^{\text {ell }}$ denote the set of pro-unipotent, elliptic regular elements of $G$.

Let $\mathcal{N}$ be an open neighbourhood of 1 in $G=\mathrm{GL}_{m}(D)$. We say that $\mathcal{N}$ is $u$ small if $\mathcal{N} \cap G_{\text {reg }}^{\text {ell }} \subset{ }_{\mathrm{u}} G_{\text {reg. }}^{\text {ell }}$. Such neighbourhoods exist. For example, the group of elements $x \in \mathrm{GL}_{m}\left(\mathfrak{o}_{D}\right)$ such that $x \equiv 1\left(\bmod \mathfrak{p}_{D}\right)$ is open in $G$ and is a $\mathrm{u}$-small neighbourhood of 1 .

Lemma. Let $G=\mathrm{GL}_{m}(D), G^{\prime}=\mathrm{GL}_{m^{\prime}}\left(D^{\prime}\right)$, for central $F$-division algebras $D$, $D^{\prime}$ such that $m^{2} \operatorname{dim} D=m^{\prime 2} \operatorname{dim} D^{\prime}$. Let $\mathcal{N}$ be a u-small open neighbourhood of 1 in $G$. There exists a u-small open neighbourhood $\mathcal{N}^{\prime}$ of 1 in $G^{\prime}$ such that every element of $\mathcal{N}^{\prime} \cap{ }_{\mathrm{u}} G_{\text {reg }}^{\text {ell }}$ is associate to an element of $\mathcal{N}$.

Proof. See 2.9 below.

We use the lemma to prove the following result, needed in 7.9 at the end of the proof of the Second Comparison Theorem (7.1). 
Proposition. Let $\left(u_{j}\right)_{j \geqslant 1}$ be a sequence of elements of ${ }_{\mathrm{u}} G_{\mathrm{reg}}^{\mathrm{ell}}$ such that $u_{j} \rightarrow 1$ as $j \rightarrow \infty$. There is a sequence of elements $u_{j}^{\prime} \in{ }_{\mathrm{u}} G^{\prime \text { reg }}$ ruch that $u_{j}^{\prime}$ is associate to $u_{j}$, for each $j$, and $u_{j}^{\prime} \rightarrow 1$.

Proof. We have to show that, if $\mathcal{N}^{\prime}$ is an open neighbourhood of 1 in $G^{\prime}$, then we may choose $u_{j}^{\prime}$ associate to $u_{j}$ such that $u_{j}^{\prime} \in \mathcal{N}^{\prime}$ for all but finitely many values of $j$. We may assume that $\mathcal{N}^{\prime}$ is u-small. By the lemma, there is a $\mathrm{u}$-small open neighbourhood $\mathcal{N}$ of 1 in $G$ such that every element of $\mathcal{N} \cap{ }_{\mathrm{u}} G_{\mathrm{reg}}^{\text {ell }}$ is associate to an element of $\mathcal{N}^{\prime}$. However, $\mathcal{N}$ contains $u_{j}$ for all but finitely many $j$.

\section{Types and cuspidal representations}

We recall material of a more algebraic nature. We work with a central simple $F$-algebra $A$ of dimension $n^{2}, n \geqslant 1$, and the group $G=A^{\times}$. We review the structure theory for the irreducible cuspidal representations of the locally profinite group $G$, as it is worked out in [38-41]. We give no detail at all, beyond establishing the vocabulary and standardizing the notation.

This analysis leads to a notion of parametric degree generalizing that of [9]. We connect it with the normalized formal degree of 1.5, to reach the starting point of the paper.

2.1. We recall standard facts concerning the algebra $A$. Most of this material can be found in, for example, [35].

Let $V$ be a simple left $A$-module. As such, $V$ is uniquely determined up to $A$-isomorphism. We define $\operatorname{End}_{A}(V)$ so that $V$ is a right $\operatorname{End}_{A}(V)$-module. The $F$-algebra $D=\operatorname{End}_{A}(V)$ is a division algebra. The given action of $A$ on $V$ induces an isomorphism $A \cong \operatorname{End}_{D}(V)$ of $F$-algebras ${ }^{1}$.

Let $V$ have dimension $m$ as $D$-vector space. The choice of a $D$-basis of $V$ induces an isomorphism $A \cong \mathrm{M}_{m}(D)$. It follows that $\operatorname{dim}_{F} D=d^{2}$, where $m d=n$, and $\operatorname{dim}_{F} V=n d$. The choice of a basis, and hence of an isomorphism $A \cong \mathrm{M}_{m}(D)$, enables us to view $D$ as embedded in $A$ as the algebra of "scalar matrices" $\operatorname{diag}(x, x, \ldots, x), x \in D$. This embedding does, of course, vary with the choice of basis.

\footnotetext{
${ }^{1}$ The authors of [38-41] prefer to have $\operatorname{End}_{A}(V)$ acting on the left, so $D$ here corresponds to the opposite of $D$ in those papers.
} 
We mention some basic facts concerning subfields of $A$. Let $E \supset F$ be a subfield of $A$. The degree $[E: F]$ thus divides $n$. Conversely, any field extension $E / F$, of degree dividing $n$, is isomorphic to an $F$-subalgebra of $A$. Any two $F$-embeddings $E \rightarrow A$ are $G$-conjugate.

(2.1.1) Let $E / F$ be a subfield of $A$, of degree $r$, and let $B$ denote the A-centralizer of $E$.

(1) The algebra $B$ is a central simple E-algebra of E-dimension $n^{2} / r^{2}$.

(2) There is an E-isomorphism $B \cong \mathrm{M}_{l}(C)$, where $C$ is a central E-division algebra of dimension $c^{2}$, such that $c=d / \operatorname{gcd}(d, r)$ and $l c=n / r$.

The assertion concerning the value of $c$ follows from [35] Theorem 31.9 (or see [44]).

2.2. We make summary remarks (see [35] or [7]) concerning the class of hereditary $\mathfrak{o}_{F}$-orders in $A$.

We view $V$ as a right $D$-vector space. An $\mathfrak{o}_{D}$-lattice in $V$ is a finitely generated $\mathfrak{o}_{D}$-submodule of $V$ which spans $V$ over $D$ (or $F$ ).

Let $\mathcal{L}$ be an $\mathfrak{o}_{D^{-}}$-lattice chain in $V$. Thus $\mathcal{L}$ is a non-empty set of right $\mathfrak{o}_{D^{-}}$ lattices in $V$, linearly ordered by inclusion and stable under translation by $D^{\times}$. It admits an enumeration $\mathcal{L}=\left\{L_{i}: i \in \mathbb{Z}\right\}$, with the following properties.

(1) $L_{i} \supseteq L_{i+1}, i \in \mathbb{Z}$, and

(2) there exists an integer $e \geqslant 1$ such that $L_{i+e}=L_{i} \mathfrak{p}_{D}, i \in \mathbb{Z}$.

The integer $e=e_{D}(\mathcal{L})$ is the $D$-period of $\mathcal{L}$.

For $j \in \mathbb{Z}$, put

$$
\mathfrak{A}_{j}(\mathcal{L})=\left\{x \in A: x L_{i} \subset L_{i+j}, i \in \mathbb{Z}\right\}
$$

The set $\mathfrak{A}=\mathfrak{A}_{0}(\mathcal{L})$ is a ring, and an $\mathfrak{o}_{F}$-order in $A$. It is indeed a hereditary $\mathfrak{o}_{F}$-order, in the sense of [35]. All such orders arise, in this way, from lattice chains in $V$.

The set $\mathfrak{P}=\mathfrak{A}_{1}(\mathcal{L})$ is the Jacobson radical of $\mathfrak{A}$ (we use the notation $\mathfrak{P}=$ $\operatorname{rad} \mathfrak{A})$ and $\mathfrak{A}_{j}(\mathcal{L})=\mathfrak{P}^{j}$ (with a suitable interpretation in the case $j<0$ ). 
The lattice chain $\mathcal{L}$ is the set of all $\mathfrak{A}$-lattices in $V$. We use the alternative notation $e\left(\mathfrak{A} \mid \mathfrak{o}_{D}\right)=e_{D}(\mathcal{L})$. We will also use the notation $e\left(\mathfrak{A} \mid \mathfrak{o}_{F}\right)$ for $F$-period of $\mathcal{L}$, defined by $\mathfrak{p}_{F} \mathfrak{A}=\mathfrak{P}^{e\left(\mathfrak{A} \mid \mathfrak{o}_{F}\right)}$. As $\mathfrak{p}_{F} \mathfrak{o}_{D}=\mathfrak{p}_{D}^{d}$, we have $e\left(\mathfrak{A} \mid \mathfrak{o}_{F}\right)=e\left(\mathfrak{A} \mid \mathfrak{o}_{D}\right) d$.

We set $U_{\mathfrak{A}}=U_{\mathfrak{A}}^{0}=\mathfrak{A}^{\times}$and $U_{\mathfrak{A}}^{j}=1+\mathfrak{P}^{j}, j \geqslant 1$. These groups $U_{\mathfrak{A}}^{j}, j \geqslant 0$, are compact open subgroups of $G$. We shall also need the group

$$
\mathcal{K}_{\mathfrak{A}}=\left\{g \in G: g \mathfrak{A} g^{-1}=\mathfrak{A}\right\} .
$$

This is an open subgroup of $G$, compact modulo the centre $F^{\times}$of $G$. It normalizes all of the groups $U_{\mathfrak{A}}^{k}, k \geqslant 0$. Writing $\mathfrak{A}=\mathfrak{A}_{0}(\mathcal{L})$ as above, we can also view $\mathcal{K}_{\mathfrak{A}}$ as the group of $g \in G$ for which $g \mathcal{L}=\mathcal{L}$.

The group $\mathcal{K}_{\mathfrak{A}}$ comes with a canonical homomorphism $v_{\mathfrak{A}}: \mathcal{K}_{\mathfrak{A}} \rightarrow \mathbb{Z}$ defined by the equivalent conditions $g \mathfrak{A}=\mathfrak{A} g=\mathfrak{P}^{v_{\mathfrak{A}}(g)}$ or $g L_{i}=L_{i+v_{\mathfrak{A}}(g)}$, for $g \in \mathcal{K}_{\mathfrak{A}}$ and $i \in \mathbb{Z}$. The homomorphism $v_{\mathfrak{A}}: \mathcal{K}_{\mathfrak{A}} \rightarrow \mathbb{Z}$ is surjective if and only if there exists $x \in \mathcal{K}_{\mathfrak{A}}$ such that $\mathfrak{P}=x \mathfrak{A}=\mathfrak{A} x$. Hereditary orders with this property are called principal orders [7].

Remark. Since we give no proofs in this area, we have no need of the notion of a "lattice sequence" in $V$, or the associated non-standard filtration of $U_{\mathfrak{A}}$, which plays such an important rôle in [38-41]. Indeed, we only ever deal with the standard filtration $\left(U_{\mathfrak{A}}^{j}\right)$ of $U_{\mathfrak{A}}$, and the hereditary order $\mathfrak{A}$ will almost always be principal.

2.3. We combine the considerations of the preceding two paragraphs. Let $\mathfrak{A}$ be a hereditary $\mathfrak{o}_{F}$-order in $A$ and $E / F$ a subfield of $A$. We say that $\mathfrak{A}$ is $E$-pure if $E^{\times} \subset \mathcal{K}_{\mathfrak{A}}$.

We recall [41] 1.7:

(2.3.1) Let $E / F$ be a subfield of $A$ and let $B$ denote the $A$-centralizer of $E$.

(1) Let $\mathfrak{A}$ be an E-pure hereditary $\mathfrak{o}_{F}$-order in $A$. The set $\mathfrak{B}=\mathfrak{A} \cap B$ is a hereditary $\mathfrak{o}_{E}$-order in $B$ and $\mathfrak{Q}=\mathfrak{P} \cap B$ is the Jacobson radical of $\mathfrak{B}$.

(2) Let $\mathfrak{B}$ be a hereditary $\mathfrak{o}_{E}$-order in $B$ and put $\mathfrak{Q}=\operatorname{rad} \mathfrak{B}$. There is a unique pair $(\rho, \mathfrak{A})$, consisting of an integer $\rho \geqslant 1$ and an $E$-pure hereditary $\mathfrak{o}_{F}$-order in $A$, such that $\mathfrak{P}^{k} \cap B=\mathfrak{Q}^{k^{\prime}}, k \in \mathbb{Z}$, where $\mathfrak{P}=\operatorname{rad} \mathfrak{A}$ and $k^{\prime}$ is the least integer $\geqslant k / \rho$. Moreover, $\mathcal{K}_{\mathfrak{B}}=\mathcal{K}_{\mathfrak{A}} \cap B$. 
If $\mathfrak{A}$ is any $E$-pure hereditary order in $A$, it has become common to refer to $\mathfrak{B}=\mathfrak{A} \cap B$ as the "trace" of $\mathfrak{A}$, and to $\mathfrak{A}$ as a "continuation" of $\mathfrak{B}$ to $A$. If we start from $\mathfrak{B}$, we refer to the order $\mathfrak{A}$, given by (2.3.1)(2), as the canonical continuation of $\mathfrak{B}$ to $A$.

A special case is worthy of overt mention: see [5] for details.

(2.3.2) Suppose, in the situation of $(2.3 .1)(2)$, that the $\mathfrak{o}_{E}$-order $\mathfrak{B}$ is maximal. The canonical continuation $\mathfrak{A}$ of $\mathfrak{B}$ is then the unique E-pure hereditary $\mathfrak{o}_{F}$-order in $A$ intersecting $B$ in $\mathfrak{B}$. The order $\mathfrak{A}$ is, moreover, maximal among E-pure hereditary orders in $A$.

In the situation of (2.3.2), the orders $\mathfrak{B}, \mathfrak{A}$, are both principal.

2.4. We examine the irreducible cuspidal representations of $G$, starting with an extreme case. Let $\mathfrak{A}$ be a maximal $\mathfrak{o}_{F}$-order in $A$. The order $\mathfrak{A}$ is therefore given by an $\mathfrak{o}_{D}$-lattice chain of the form $\mathcal{L}=\left\{L \mathfrak{p}_{D}^{i}: i \in \mathbb{Z}\right\}$, for a fixed $\mathfrak{o}_{D}$-lattice $L$ in $V$. Any two maximal orders in $A$ are $G$-conjugate.

We may identify $A$ with $\mathrm{M}_{m}(D)$ via a basis of $V$, as in 2.1 , chosen to be an $\mathfrak{o}_{D}$-basis of $L$. The order $\mathfrak{A}$ then becomes identified with $\mathrm{M}_{m}\left(\mathfrak{o}_{D}\right)$. Taking the case $\mathfrak{A}=\mathrm{M}_{m}\left(\mathfrak{o}_{D}\right)$ and viewing $D$ as the algebra of scalar matrices in $A$ (as in 2.1 ), we get

$$
\begin{aligned}
\operatorname{rad} \mathfrak{A}=\mathfrak{p}_{D} \mathfrak{A} & =\mathfrak{A p}_{D}=\mathrm{M}_{m}\left(\mathfrak{p}_{D}\right), \\
\mathcal{K}_{\mathfrak{A}} & =D^{\times} U_{\mathfrak{A}} .
\end{aligned}
$$

We conclude that $U_{\mathfrak{A}} / U_{\mathfrak{A}}^{1} \cong \mathrm{GL}_{m}\left(\mathbb{k}_{D}\right)$. If $\varpi_{D}$ is a prime element of $D$, viewed as an element of $\mathcal{K}_{\mathfrak{A}}$, then $\varpi_{D}$ acts (by conjugation) on this group. The action is that of a generator of $\operatorname{Gal}\left(\mathbb{k}_{D} / \mathbb{k}_{F}\right)$ acting on matrix coefficients.

Definition 1. A maximal simple type of level zero in $G$ is a pair $(U, \lambda)$, where

(1) $U=U_{\mathfrak{A}}$, for a maximal $\mathfrak{o}_{F}$-order $\mathfrak{A}$ in $A$, and

(2) $\lambda$ is an irreducible representation of $U_{\mathfrak{A}}$, trivial on $U_{\mathfrak{A}}^{1}$ and inflated from an irreducible cuspidal representation of the finite group $U_{\mathfrak{A}} / U_{\mathfrak{A}}^{1} \cong \mathrm{GL}_{m}\left(\mathbb{k}_{D}\right)$.

Let $(U, \lambda), U=U_{\mathfrak{A}}$, be a maximal simple type of level zero in $G$. The following lemma is a straightforward instance of the more general considerations of [24]. However, it can equally be proved by a direct calculation, essentially identical to that in the split case $G=\mathrm{GL}_{n}(F)$. 


\section{Lemma.}

(1) Let $\tau$ be an irreducible representation of $U=U_{\mathfrak{A}}$, trivial on $U_{\mathfrak{A}}^{1}$. If $\tau$ intertwines with $\lambda$ in $G$, there exists $x \in \mathcal{K}_{\mathfrak{A}}$ such that $\tau^{x} \cong \lambda$.

(2) Let $\boldsymbol{J}(\lambda)$ denote the set of elements of $G$ which intertwine the representation $\lambda$, let $w_{\lambda}$ be the number of distinct $\mathcal{K}_{\mathfrak{A}}$-conjugates of $\lambda$. The set $\boldsymbol{J}(\lambda)$ is then the unique subgroup of $\mathcal{K}_{\mathfrak{A}}$ of index $w_{\lambda}$ and containing $F^{\times} U_{\mathfrak{A}}$. The group $\boldsymbol{J}(\lambda)$ normalizes the pair $(U, \lambda)$.

The integer $w_{\lambda}$ may also be described as follows. The representation $\lambda$ is inflated from an irreducible cuspidal representation $\tilde{\lambda}$ of $\mathrm{GL}_{m}\left(\mathbb{k}_{D}\right)$, via an isomorphism $U_{\mathfrak{A}} / U_{\mathfrak{A}}^{1} \cong \mathrm{GL}_{m}\left(\mathbb{k}_{D}\right)$. The number of distinct conjugates of $\tilde{\lambda}$, under the natural action of $\operatorname{Gal}\left(\mathbb{k}_{D} / \mathbb{k}_{F}\right)$, is then $w_{\lambda}$.

The group $\mathcal{K}_{\mathfrak{A}} / F^{\times} U_{\mathfrak{A}}$ is cyclic of order $d$, so $w_{\lambda}$ divides $d$. In this situation, we define the parametric degree $\delta_{0}(\lambda)$ of $\lambda$ by

$$
\delta_{0}(\lambda)=m w_{\lambda}
$$

Remark 1. In practice, it is usually more convenient to think of $\tilde{\lambda}$ as being given, via the Green parametrization, by a character $\chi$ of $\boldsymbol{l}^{\times}$, where $\boldsymbol{l} / \mathbb{k}_{D}$ is a field extension of degree $m$ contained in $\mathrm{M}_{m}\left(\mathbb{k}_{D}\right)$. Since $\tilde{\lambda}$ is cuspidal, the character $\chi$ is $\boldsymbol{l} / \mathbb{k}_{D}$-regular, in that its conjugates $\chi^{\sigma}, \sigma \in \mathrm{Gal}\left(\boldsymbol{l} / \mathbb{k}_{D}\right)$ are distinct. Moreover,

$$
\operatorname{tr} \tilde{\lambda}(x)=(-1)^{m-1} \sum_{\sigma \in \operatorname{Gal}\left(\boldsymbol{l} / \mathbb{k}_{D}\right)} \chi^{\sigma}(x),
$$

for every $\boldsymbol{l} / \mathbb{k}_{D}$-regular element $x$ of $\boldsymbol{l}^{\times}$. The number of distinct conjugates of $\chi$ under $\operatorname{Gal}\left(\boldsymbol{l} / \mathbb{k}_{F}\right)$ is then $\delta_{0}(\lambda)=m w_{\lambda}$. (For the Green parametrization, see [25] or [31], although the authors find the character tables in [34] particularly convenient. The summary in [14] $\S 2$ will be adequate for the purposes of this paper.)

We note the following straightforward consequence of the lemma.

Proposition. Let $(U, \lambda)$ be a maximal simple type in $G$ of level zero.

(1) The representation $\lambda$ admits extension to a representation $\Lambda$ of the group $\boldsymbol{J}(\lambda)$. 
(2) The representation $\Lambda$ is uniquely determined up to twisting with a character of the form $\chi_{A}=\chi \circ \operatorname{Nrd}_{A}$, where $\chi$ is an unramified character of $F^{\times}$.

(3) Let $\chi$ be an unramified character of $F^{\times}$. The representations $\Lambda$ and $\chi_{A} \otimes \Lambda$ intertwine in $G$ if and only if they are equivalent. Moreover, $\Lambda \cong \chi_{A} \otimes \Lambda$ if and only if $\chi^{\delta_{0}(\lambda)}=1$.

(4) The representation

$$
\pi_{\Lambda}=c-\operatorname{Ind}_{\boldsymbol{J}(\lambda)}^{G} \Lambda
$$

of $G$ is irreducible and cuspidal.

Representations $(\boldsymbol{J}(\lambda), \Lambda)$, of this form, constitute the class of extended maximal simple types of level zero in $G$.

Let $\pi$ be an irreducible cuspidal representation of $G$. One says that $\pi$ has level zero if it admits a non-zero vector fixed by $U_{\mathfrak{A}}^{1}$, for some maximal $\mathfrak{o}_{F}$-order $\mathfrak{A}$ in $A$. The main result in this area is:

Theorem. Let $\pi$ be an irreducible cuspidal representation of $G$, of level zero.

(1) The representation $\pi$ contains a maximal simple type $(U, \lambda)$ of level zero.

(2) There exists an extended maximal simple type $(\boldsymbol{J}(\lambda), \Lambda)$ such that $\Lambda \mid$ $U \cong \lambda$ and $\pi \cong \pi_{\Lambda}$.

(3) The representation $\pi$ determines both $(U, \lambda)$ and $(\boldsymbol{J}(\lambda), \Lambda)$ uniquely, up to $G$-conjugacy.

(4) The number $t(\pi)$ of unramified characters $\chi$ of $F^{\times}$satisfying $\chi \pi \cong \pi$ is given by $t(\pi)=\delta_{0}(\lambda)$.

The proof is comparatively straightforward, mimicking the corresponding result in the split case. It can again be regarded as a simple instance of [24].

Definition 2. Let $\pi$ be an irreducible cuspidal representation of $G \cong \mathrm{GL}_{m}(D)$, of level zero. The parametric degree $\delta(\pi)$ of $\pi$ is $\delta_{0}(\lambda)$, where $(U, \lambda)$ is a maximal simple type contained in $\pi$.

Part (4) of the theorem implies that the definition of $\delta(\pi)$ is independent of the choice of type $\lambda$.

Remark 2. In the split case $G=\mathrm{GL}_{n}(F)$, an irreducible cuspidal representation of $G$ (of level zero) always satisfies $\delta(\pi)=n$. This, however, fails for general 
groups $\mathrm{GL}_{m}(D)$. If $\pi$ is an irreducible cuspidal representation of $\mathrm{GL}_{m}(D)$ of level zero, then $m$ divides $\delta(\pi)$ and $\delta(\pi)$ divides $n$, but $\delta(\pi)$ is subject to some further combinatorial constraints. For, let $\boldsymbol{l} / \mathbb{k}_{D}$ be a field extension of degree $m$ as above, and write $\Gamma=\operatorname{Gal}\left(\boldsymbol{l} / \mathbb{k}_{F}\right), \Delta=\operatorname{Gal}\left(\boldsymbol{l} / \mathbb{k}_{D}\right)$. Let $\chi$ be a character of $l^{\times}$, and let $\Phi$ be the subgroup of $\Gamma$ which fixes $\chi$. The character $\chi$ gives rise to a maximal simple type if and only if $\Phi \cap \Delta$ is trivial. In other words, if we fix a positive integer $\delta$, divisible by $m$ and dividing $n$, there exists an irreducible cuspidal representation $\pi$ of $G$, of level zero and with $\delta(\pi)=\delta$, if and only if $n / \delta$ is relatively prime to $m$.

2.5. We proceed to the general case. We follow [38-40] and, particularly, the summary in [41]. We abbreviate the treatment as much as possible, while keeping the terminology and notation strictly parallel to those of [17].

Let $E / F$ be a finite field extension, and let $\beta \in E^{\times}$. We assume throughout that $v_{E}(\beta)<0$ and that $E=F[\beta]$. Let $A$ be a central simple $F$-algebra as before and let $\iota: E \rightarrow A$ be an $F$-embedding. Let $\mathfrak{A}$ be an $\iota E$-pure hereditary $\mathfrak{o}_{F}$-order in $A$. The period $e_{\mathfrak{A}}=e\left(\mathfrak{A} \mid \mathfrak{o}_{F}\right)=\operatorname{de}\left(\mathfrak{A} \mid \mathfrak{o}_{D}\right)$ is divisible by $e(E \mid F)$ and $v_{\mathfrak{A}}(\boldsymbol{\iota} \beta)=e_{\mathfrak{A}} v_{E}(\beta) / e(E \mid F)$. Any quadruple $\left[\mathfrak{A}, \ell_{\mathfrak{A}}, r, \boldsymbol{\iota} \beta\right]$, with $\ell_{\mathfrak{A}}=-v_{\mathfrak{A}}(\boldsymbol{\iota} \beta)$ and $r<\ell_{\mathfrak{A}}$, is then a pure stratum in $A$.

Attached to the pure stratum $\left[\mathfrak{A}, \ell_{\mathfrak{A}}, r, \boldsymbol{\iota} \beta\right]$ is the critical exponent $k_{0}(\iota \beta, \mathfrak{A}) \in$ $\mathbb{Z} \cup\{-\infty\}$. By definition, $\left[\mathfrak{A}, \ell_{\mathfrak{A}}, r, \boldsymbol{\iota} \beta\right]$ is simple if $-k_{0}(\boldsymbol{\iota} \beta, \mathfrak{A})>r$. The critical exponent satisfies

$$
e(E \mid F) k_{0}(\iota \beta, \mathfrak{A})=e_{\mathfrak{A}} k_{0}(\beta, \mathfrak{A}(E)),
$$

where $\mathfrak{A}(E)$ is the unique $E$-pure hereditary $\mathfrak{o}_{F}$-order in the algebra $\operatorname{End}_{F}(E)$.

We henceforward assume that $\left[\mathfrak{A}, \ell_{\mathfrak{A}}, 0, \boldsymbol{\iota} \beta\right]$ is a simple stratum. We identify $E$ with its image in $A$ and suppress the notation $\iota$. The stratum gives rise to a pair

$$
\mathfrak{H}(\beta, \mathfrak{A}) \subset \mathfrak{J}(\beta, \mathfrak{A}) \subset \mathfrak{A}
$$

of $\mathfrak{o}_{F}$-orders in $A$. We take the standard filtration subgroups of unit groups

$$
\begin{aligned}
H^{k}(\beta, \mathfrak{A}) & =\mathfrak{H}(\beta, \mathfrak{A}) \cap U_{\mathfrak{A}}^{k}=\mathfrak{H}(\beta, \mathfrak{A})^{\times} \cap U_{\mathfrak{A}}^{k}, \\
J^{k}(\beta, \mathfrak{A}) & =\mathfrak{J}(\beta, \mathfrak{A}) \cap U_{\mathfrak{A}}^{k}=\mathfrak{J}(\beta, \mathfrak{A})^{\times} \cap U_{\mathfrak{A}}^{k},
\end{aligned}
$$

for $k \in \mathbb{Z}, k \geqslant 0$. 
We choose, once for all, a character $\psi_{F}$ of $F$ which is "of level one". That is to say, $\mathfrak{p}_{F} \subset \operatorname{Ker} \psi_{F}$ but $\psi_{F} \mid \mathfrak{o}_{F} \neq 1$. We use $\psi_{F}$ to construct the finite set $\mathcal{C}\left(\mathfrak{A}, \beta, \psi_{F}\right)$ of so-called simple characters of the group $H^{1}(\beta, \mathfrak{A})$.

Comment. The definitions in [38] of the orders $\mathfrak{H}, \mathfrak{J}$, and the simple character set $\mathcal{C}$ are necessarily indirect. However, as pointed out in [38] Propositions 3.42 and 3.45 , one may see ex post facto that the obvious generalization of the approach of [17], via simple approximations to pure strata, applies without significant change. When we need to enter into more detail, this is the method we shall adopt. We therefore say no more at this stage concerning the constructions in $[38]$.

We summarize some of the main points.

(2.5.1) Let $B$ denote the $A$-centralizer of $E$, let $\mathfrak{B}=\mathfrak{A} \cap B$, and let $\theta \in$ $\mathcal{C}\left(\mathfrak{A}, \beta, \psi_{F}\right)$. Abbreviate $J^{k}=J^{k}(\beta, \mathfrak{A})$, and so on.

(1) We have $J^{0}=U_{\mathfrak{B}} J^{1}$ and $U_{\mathfrak{B}} \cap J^{1}=U_{\mathfrak{B}}^{1}$.

(2) There exists a unique irreducible representation $\eta_{\theta}$ of $J^{1}$ such that $\eta_{\theta} \mid H^{1}$ contains $\theta$.

(3) For $g \in G$, the following are equivalent:

(a) $g$ intertwines $\theta$;

(b) $g$ intertwines $\eta_{\theta}$;

(c) $g \in J^{1} B^{\times} J^{1}$.

(4) If $g \in J^{1} B^{\times} J^{1}$, the coset $J^{1} g J^{1}$ supports a one-dimensional space of intertwining operators for the representation $\eta_{\theta}$.

We need to delineate more carefully a phenomenon underlying parts of (2.5.1). The quotient

$$
\mathfrak{V}(\beta, \mathfrak{A})=\mathfrak{J}^{1}(\beta, \mathfrak{A}) / \mathfrak{H}^{1}(\beta, \mathfrak{A})=J^{1} / H^{1}
$$

is a vector space over $\mathbb{k}_{E}$ and hence an elementary abelian $p$-group. It has a sequence of subspaces

$$
\mathfrak{V}^{k}(\beta, \mathfrak{A})=J^{k} / H^{k}, \quad k \geqslant 1
$$

For $x, y \in J^{k}(\beta, \mathfrak{A})$, the commutator $[x, y]=x y x^{-1} y^{-1}$ lies in $H^{2 k}$, and we consider the pairing $h_{\theta}:(x, y) \mapsto \theta[x, y]$. The values of this pairing are $p$-th 
roots of unity, and $h_{\theta}$ defines an alternating bilinear form

$$
h_{\theta}: \mathfrak{V}^{k}(\beta, \mathfrak{A}) \times \mathfrak{V}^{k}(\beta, \mathfrak{A}) \longrightarrow \mathbb{F}_{p}, \quad k \geqslant 1,
$$

over $\mathbb{F}_{p}$. We then have [38] Théorème 3.52:

(2.5.4) The alternating form (2.5.3) is nondegenerate, for every $k \geqslant 1$. As alternating space, $\mathfrak{V}(\beta, \mathfrak{A})$ is the orthogonal sum of nondegenerate alternating spaces isometric to $\mathfrak{V}^{k}(\beta, \mathfrak{A}) / \mathfrak{V}^{k+1}(\beta, \mathfrak{A})=J^{k} / J^{k+1} H^{k}, k \geqslant 1$.

For the next step, we need a simplified version of the main result of [39]. As before, $[\mathfrak{A}, \ell, 0, \beta]$ is a simple stratum in $A$ and $\theta \in \mathfrak{C}\left(\mathfrak{A}, \beta, \psi_{F}\right)$. Again, $B$ is the $A$-centralizer of $E=F[\beta]$ and $\mathfrak{B}=\mathfrak{A} \cap B$.

(2.5.5) There exists an irreducible representation $\kappa$ of $J=J^{0}(\beta, \mathfrak{A})$ such that

(1) $\kappa \mid J^{1} \cong \eta_{\theta}$, and

(2) $\kappa$ is intertwined by every element of $B^{\times}$.

These conditions determine $\kappa$ uniquely, up to tensoring with a character of $U_{\mathfrak{B}} / U_{\mathfrak{B}}^{1}=J / J^{1}$ of the form $\chi \circ \operatorname{Nrd}_{B}$, where $\chi$ is a character of $U_{E}$ trivial on $U_{E}^{1}$.

We call $\kappa$ a wide extension ${ }^{2}$ of $\eta_{\theta}$. Since $\operatorname{dim} \kappa$ has $p$-power dimension, two wide extensions $\kappa_{i}$ of $\eta_{\theta}$ are equivalent if and only if the $p$-regular parts of the characters $\operatorname{det} \kappa_{i}$ are the same.

2.6. We use a special case of the foregoing to make a fundamental definition.

Definition. A maximal simple type of positive level in $G$ is a pair $(J, \lambda)$ constructed as follows.

(1) There is a simple stratum $[\mathfrak{A}, \ell, 0, \beta]$ in $A$, such that $J=J^{0}(\beta, \mathfrak{A})$, satisfying the following condition: if $B$ is the A-centralizer of the field $E=F[\beta]$ and $\mathfrak{B}=\mathfrak{A} \cap B$, then $\mathfrak{B}$ is a maximal $\mathfrak{o}_{E}$-order in $B$.

(2) There is a simple character $\theta \in \mathcal{C}\left(\mathfrak{A}, \beta, \psi_{F}\right)$ and a maximal simple type $\left(U_{\mathfrak{B}}, \sigma_{\mathfrak{B}}\right)$ of level zero in $B^{\times}$such that $\lambda$ is a representation of $J$ of the form

$$
\lambda=\sigma \otimes \kappa,
$$

\footnotetext{
${ }^{2}$ This term is intended to replace the arcane expression " $\beta$-extension" surviving from [17].
} 
where

(a) $\kappa$ is a wide extension of $\eta_{\theta}$, and

(b) $\sigma$ is the representation of $J$, trivial on $J^{1}$, deduced from $\sigma_{\mathfrak{B}}$ via the isomorphism $U_{\mathfrak{B}} / U_{\mathfrak{B}}^{1} \cong J / J^{1}$.

Using the same notation, we note a particular property given by [40] Proposition 4.3 .

(2.6.1) Let $g \in G$. The following conditions are equivalent:

(1) $g$ intertwines $\lambda$;

(2) $g$ normalizes $J$ and $\lambda^{g} \cong \lambda$;

(3) $g \in \boldsymbol{J}_{B}\left(\sigma_{\mathfrak{B}}\right) J^{1}$, where $\boldsymbol{J}_{B}\left(\sigma_{\mathfrak{B}}\right)$ is the normalizer of the simple type $\left(U_{\mathfrak{B}}, \sigma_{\mathfrak{B}}\right)$ in $B^{\times}$.

We write $\boldsymbol{J}(\lambda)=\boldsymbol{J}_{B}\left(\sigma_{\mathfrak{B}}\right) J^{1}$.

We define the parametric degree $\delta_{0}(\lambda)$ of the maximal simple type $(J, \lambda)$ by

$$
\delta_{0}(\lambda)=\delta_{0}\left(\sigma_{\mathfrak{B}}\right)[E: F] .
$$

One may show directly that $\delta_{0}(\lambda)$ does indeed depend only on the isomorphism class of the pair $(J, \lambda)$, and not on the simple stratum and simple character we chose to describe it. There is no need to enter into the detail, since it is a consequence of 2.7 Proposition below.

When speaking of a maximal simple type in $G$, we mean either a maximal simple type of level zero or a maximal simple type of positive level.

We now extend the definition as before. We recall that, if $\chi$ is a character of $F^{\times}$, then $\chi_{A}$ denotes the character $\chi \circ \operatorname{Nrd}_{A}$ of $G$.

(2.6.2) Let $(J, \lambda)$ be a maximal simple type in $G$, as in the Definition.

(1) The representation $\lambda$ admits extension to a representation $\Lambda$ of $\boldsymbol{J}(\lambda)$.

(2) The set of such extensions consists of the representations $\chi_{A} \otimes \Lambda$, where $\chi$ ranges over the unramified characters of $F^{\times}$.

(3) The representation $\chi_{A} \otimes \Lambda$ intertwines with $\Lambda$ if and only if it is equivalent to $\Lambda$. The group of unramified characters $\chi$ satisfying $\chi_{A} \otimes \Lambda \cong \Lambda$ has order $\delta_{0}\left(\sigma_{\mathfrak{B}}\right) f(E \mid F)$. 
(4) The representation of $G$ defined by

$$
\pi_{\Lambda}=c-\operatorname{Ind}_{\boldsymbol{J}(\lambda)}^{G} \Lambda
$$

is irreducible and cuspidal. Moreover,

(a) if $\chi$ is an unramified character of $F^{\times}$, then $\pi_{\chi_{A} \otimes \Lambda} \cong \chi \pi_{\Lambda}$, and

(b) $t\left(\pi_{\Lambda}\right)=\delta_{0}\left(\sigma_{\mathfrak{B}}\right) f(E \mid F)$.

A pair of the form $(\boldsymbol{J}(\lambda), \Lambda)$, obtained from a maximal simple type $(J, \lambda)$ as in (2.6.2), is called an extended maximal simple type of positive level in $G$. We can now complete the picture with the following, which is the main result of [41].

Theorem. Let $\pi$ be an irreducible cuspidal representation of $G$, not of level zero. There is a maximal simple type $(J, \lambda)$ in $G$, of positive level, such that $\pi$ contains $\lambda$. Consequently, there exists an extended maximal simple type $(\boldsymbol{J}, \Lambda)$ in $G$ such that $\pi \cong c-\operatorname{Ind}_{J}^{G} \Lambda$.

2.7. Let $\pi$ be an irreducible cuspidal representation of $G=A^{\times} \cong \mathrm{GL}_{m}(D)$. Attached to $\pi$ we have the numerical invariant $t(\pi)$ (as in 1.4). Comparing central characters, we see that $t(\pi)$ divides $n$. The representation $\pi$ contains a maximal simple type $(J, \lambda)$, the parametric degree $\delta_{0}(\lambda)$ of which also divides $n$, and $t(\pi)$ divides $\delta_{0}(\lambda)$.

Proposition. Let $\pi$ be an irreducible cuspidal representation of $G \cong \mathrm{GL}_{m}(D)$. If $\left(J_{i}, \lambda_{i}\right), i=1,2$, are maximal simple types occurring in $\pi$, then $\delta_{0}\left(\lambda_{1}\right)=$ $\delta_{0}\left(\lambda_{2}\right)$.

Proof. We need an auxiliary result. To state it, we need another notation. If $a, b$ are positive rational numbers, we write $a \equiv b\left(\bmod p^{\mathbb{Z}}\right)$ to mean that $a b^{-1}$ is a power (positive or negative) of $p$. We choose a Haar measure $\dot{\mu}$ on $G / F^{\times}$, with the property that $\dot{\mu}\left(P F^{\times} / F^{\times}\right)$is an integral power of $p$, for any open pro- $p$ subgroup $P$ of $G$.

Lemma. Let $(J, \lambda)$ be a maximal simple type in $G$. If it has positive level and is based on a simple stratum $[\mathfrak{A}, \ell, 0, \beta]$ (as in 2.6 Definition,), set $E=F[\beta]$. If it has level zero, set $E=F$.

If $\tau$ is an irreducible representation of $G$ containing $\lambda$, then $\tau$ is cuspidal and

$$
d(\tau, \dot{\mu}) \equiv \frac{t(\tau)}{n} \frac{q-1}{q^{n / e(E \mid F)}-1} \quad\left(\bmod p^{\mathbb{Z}}\right) .
$$


Proof. If $\tau$ is an irreducible representation of $G$ containing $\lambda$, then

$$
\tau \cong c-\operatorname{Ind}_{\boldsymbol{J}(\lambda)}^{G} \Theta
$$

for an irreducible representation $\Theta$ of $\boldsymbol{J}(\lambda)$ extending $\lambda$. Indeed, $(\boldsymbol{J}(\lambda), \Theta)$ is an extended maximal simple type. Theorem A.14 of [8] shows that the formal degree $d(\tau, \dot{\mu})$ of $\pi$ is given by

$$
d(\tau, \dot{\mu})=\frac{\operatorname{dim} \Theta}{\dot{\mu}(\boldsymbol{J}(\lambda))}=\frac{\operatorname{dim} \lambda}{\dot{\mu}(\boldsymbol{J}(\lambda))} .
$$

(As remarked in $\S 1$, the proof of this result in [8] applies unchanged to our present situation.) We evaluate this expression to sufficient accuracy.

Suppose, for the moment, that we are in the positive level case and use the notation of the definition in 2.6. We have $\operatorname{dim} \Theta=\operatorname{dim} \lambda=\operatorname{dim} \sigma_{\mathfrak{B}} \cdot \operatorname{dim} \kappa$, and $\operatorname{dim} \kappa$ is a power of $p$. If $B \cong \mathrm{M}_{l}(C)$, where $C$ is a central $E$-division algebra such that $\operatorname{dim}_{E} C=c^{2}$, then $\sigma_{\mathfrak{B}}$ is the inflation of an irreducible cuspidal representation of $\mathrm{GL}_{l}\left(\mathbb{k}_{C}\right)$. Therefore

$$
\operatorname{dim} \sigma_{\mathfrak{B}} \equiv \frac{\left|\mathrm{GL}_{l}\left(\mathbb{k}_{C}\right)\right|}{q_{C}^{l}-1}\left(\bmod p^{\mathbb{Z}}\right),
$$

where $q_{C}=\left|\mathbb{k}_{C}\right|$. The group $\boldsymbol{J}(\lambda)$ contains $F^{\times} J, J=J^{0}(\beta, \mathfrak{A})$, with index $e(E \mid F) l c / \delta_{0}\left(\sigma_{\mathfrak{B}}\right)$. Thus

$$
\begin{aligned}
\dot{\mu}\left(\boldsymbol{J}(\lambda) / F^{\times}\right) & =\frac{e(E \mid F) l c}{\delta_{0}\left(\sigma_{\mathfrak{B}}\right)} \dot{\mu}\left(J / U_{F}\right) \\
& =\frac{n}{f(E \mid F) \delta_{0}\left(\sigma_{\mathfrak{B}}\right)} \dot{\mu}\left(J / U_{F}\right) \\
& =\frac{n}{t(\tau)} \dot{\mu}\left(J / U_{F}\right) .
\end{aligned}
$$

The measure factor satisfies $\dot{\mu}\left(J / U_{F}\right) \equiv\left|\mathrm{GL}_{l}\left(\mathbb{k}_{C}\right)\right| /(q-1)\left(\bmod p^{\mathbb{Z}}\right)$, giving

$$
d(\tau, \dot{\mu}) \equiv \frac{t(\tau)}{n} \frac{q-1}{q_{C}^{l}-1} \quad\left(\bmod p^{\mathbb{Z}}\right)
$$

while $q_{C}=q_{E}^{c}$, so

$$
q_{C}^{l}=q_{E}^{l c}=q_{E}^{n /[E: F]}=q^{n / e(E \mid F)} .
$$


Thus the lemma is proved when $(J, \lambda)$ has positive level. When $(J, \lambda)$ has level zero, one uses the same argument with $E=F$ and $\mathrm{GL}_{l}\left(\mathbb{k}_{C}\right)=\mathrm{GL}_{m}\left(\mathbb{k}_{D}\right)$.

We return to the statement of the proposition. If $\pi$ contains the two maximal simple types $\left(J_{i}, \lambda_{i}\right),(2.7 .1)$ implies (in the obvious notation) that $e\left(E_{1} \mid F\right)=$ $e\left(E_{2} \mid F\right)$. However, $t(\pi)=f\left(E_{i} \mid F\right) \delta_{0}\left(\sigma_{\mathfrak{B}_{i}}\right)$, and $\delta_{0}\left(\lambda_{i}\right)=\left[E_{i}: F\right] \delta_{0}\left(\sigma_{\mathfrak{B}_{i}}\right)$, whence the result follows.

We may now make a formal definition.

Definition. Let $\pi$ be an irreducible cuspidal representation of $G \cong \mathrm{GL}_{m}(D)$. The parametric degree $\delta(\pi)$ of $\pi$ is $\delta(\pi)=\delta_{0}(\lambda)$, for any maximal simple type $(J, \lambda)$ contained in $\pi$.

We have already remarked, at the beginning of the paragraph:

(2.7.3) Let $\pi$ be an irreducible cuspidal representation of $G$. The integer $\delta(\pi)$ divides $n$ and is divisible by $t(\pi)$.

2.8. We now state a result connecting the preceding material with that of $\S 1$. The proof, however, requires a distinctive family of ideas, so we have given it separate treatment in Appendix 1.

Let $G=\mathrm{GL}_{m}(D)$ as before, with $\operatorname{dim} D=d^{2}$ and $n=m d$. Take $\pi \in \mathcal{A}_{m}^{\square}(D)$. If $\pi$ is cuspidal, its parametric degree $\delta(\pi)$ has just been defined in 2.7. Otherwise, there is a positive divisor $r$ of $m$ and an irreducible cuspidal representation $\sigma$ of $\mathrm{GL}_{r}(D)$ such that the cuspidal support of $\pi$ consists of unramified twists of $\sigma$ (1.5). We set

$$
\delta(\pi)=\delta(\sigma)
$$

In this situation, we also have the relation

$$
t(\pi)=t(\sigma) .
$$

Theorem. Let $\pi \in \mathcal{A}_{m}^{\square}(D)$. The normalized formal degree $\operatorname{deg} \pi$ satisfies

$$
\operatorname{deg} \pi \equiv t(\pi)\left(q^{n}-1\right) /\left(q^{t(\pi) n / \delta(\pi)}-1\right) \quad\left(\bmod p^{\mathbb{Z}}\right) .
$$

Proof. See Appendix 1 below. 
Corollary 1. Let $\boldsymbol{j}: \mathcal{A}_{n}^{\square}(F) \rightarrow \mathcal{A}_{m}^{\square}(D)$ denote the Jacquet-Langlands correspondence. If $\pi \in \mathcal{A}_{n}^{\square}(F)$, then $\delta(\boldsymbol{j} \pi)=\delta(\pi)$.

Proof. Write $\tau=\boldsymbol{j} \pi$. We have $t(\tau)=t(\pi)$ (1.4.4) and $\operatorname{deg} \pi=\operatorname{deg} \boldsymbol{j} \pi$ (1.5 Theorem). Consequently,

$$
\left(q^{t(\pi) n / \delta(\pi)}-1\right) \equiv\left(q^{t(\pi) n / \delta(\tau)}-1\right) \quad\left(\bmod p^{\mathbb{Z}}\right),
$$

whence the assertion follows.

Let $\pi \in \mathcal{A}_{m}^{\square}(D)$. The integer $t(\pi)$ divides $\delta(\pi)$ (2.7.3). We say that $\pi$ is essentially tame if $p$ does not divide $\delta(\pi) / t(\pi)$.

Lemma. Let $\pi \in \mathcal{A}_{m}^{\square}(D)$ be cuspidal. The representation $\pi$ is essentially tame if and only if either

(a) $\pi$ has level zero, or

(b) $\pi$ has positive level and contains a maximal simple type based on a simple stratum $[\mathfrak{A}, \ell, 0, \beta]$ such that the field extension $F[\beta] / F$ is tamely ramified. A general element $\pi \in \mathcal{A}_{m}^{\square}(D)$ is essentially tame if and only if some element of its cuspidal support is essentially tame.

Proof. If $\pi$ has level zero, then $t(\pi)=\delta(\pi)$ (2.4 Theorem), and so $\pi$ is essentially tame. If $\pi$ has positive level, it contains a maximal simple type based on a simple stratum $[\mathfrak{A}, \ell, 0, \beta]$, and $\delta(\pi) / t(\pi)=e(F[\beta] \mid F)$. The final assertion follows from $(2.8 .1),(2.8 .2)$.

The proof of (b) shows that, if $\pi$ is essentially tame, then $F[\gamma] / F$ is tamely ramified for any simple stratum $\left[\mathfrak{A}^{\prime}, \ell^{\prime}, 0, \gamma\right]$ underlying a maximal simple type occurring in $\pi$.

Notation. Let $\mathcal{A}_{m}^{\mathrm{et}}(D)$ denote the set of $\pi \in \mathcal{A}_{m}^{\square}(D)$ which are essentially tame and satisfy $\delta(\pi)=n$.

All elements of $\mathcal{A}_{m}^{\text {et }}(D)$ are cuspidal and, in particular, $\mathcal{A}_{n}^{\text {et }}(F)$ is the set of equivalence classes of irreducible, essentially tame, cuspidal representations of $\mathrm{GL}_{n}(F)$. (This notation agrees with that of [11].) From Corollary 1 and (1.4.4), we obtain: 
Corollary 2. The Jacquet-Langlands correspondence $\boldsymbol{j}: \mathcal{A}_{n}^{\square}(F) \rightarrow \mathcal{A}_{m}^{\square}(D)$ induces a bijection $\mathcal{A}_{n}^{\mathrm{et}}(F) \rightarrow \mathcal{A}_{m}^{\mathrm{et}}(D)$.

Corollary 2 provides the true starting point of the paper. It is our task to render the map $\mathcal{A}_{n}^{\text {et }}(F) \rightarrow \mathcal{A}_{m}^{\text {et }}(D)$ explicit.

2.9. We prove 1.6 Lemma. For the first step, let $C$ be a central $F$-division algebra of dimension $n^{2}=m^{2} d^{2}, d^{2}=\operatorname{dim} D$, and suppose that $G^{\prime}=\mathrm{GL}_{1}(C)$. In the algebra $A=\mathrm{M}_{m}(D)$, we choose a maximal order $\mathfrak{M}$. Shrinking the given neighbourhood $\mathcal{N}$, we may assume that it takes the form $U_{\mathfrak{M}}^{k}$, for some integer $k \geqslant 1$. Set $\mathcal{N}^{\prime}=U_{C}^{m k}=1+\mathfrak{p}_{C}^{m k}$, and let $x \in \mathcal{N}^{\prime}$ be elliptic regular. Let $E=F[x]$, let $e^{\prime}=e(F[x] \mid F)$ and let $e$ be the least common multiple of $e^{\prime}$ and $d$. Since $d$ divides $e$ and $e$ divides $n$, the order $\mathfrak{M}$ contains a principal order $\mathfrak{A}$ of $F$-period $e$. There is an $F$-embedding $\phi: E \rightarrow A$ such that $\mathfrak{A}$ is $\phi(E)$-pure. We write $x=1+y$ and compare valuations:

$$
v_{\mathfrak{A}}(\phi y)=e v_{E}(y) / e^{\prime}=e v_{C}(y) / n \geqslant e m k / n=e k / d .
$$

However, $U_{\mathfrak{A}}^{e k / d} \subset U_{\mathfrak{M}}^{k}$, so $x$ is associate to an element of $\mathcal{N}$, as required.

We next treat the case where $G=\mathrm{GL}_{1}(C)$ and $G^{\prime}=\mathrm{GL}_{m}(D)$. We take $\mathcal{N}$ of the form $U_{C}^{m k}$, for some $k \geqslant 1$. We fix a maximal order $\mathfrak{M}$ in $\mathrm{M}_{m}(D)$ as before, and set $\mathcal{N}^{\prime}=U_{\mathfrak{M}}^{k}$. If $x=1+y \in \mathcal{N}^{\prime}$ is elliptic regular, we put $E=F[x]$ and define $e^{\prime}=e(E \mid F)$ in the same way. We have

$$
v_{E}(y) \geqslant e^{\prime} v_{\mathfrak{M}}(y) / d \geqslant e^{\prime} k / d .
$$

If we choose an $F$-embedding $\phi: E \rightarrow C$, we get

$$
v_{C}(\phi y)=n v_{E}(y) / e^{\prime} \geqslant k n / d=m k .
$$

It follows that $x$ is associate to an element of $\mathcal{N}$, as required.

The general case of the proposition now follows from a simple transitivity argument.

\section{Tame ramification}

Let $A$ be a central simple $F$-algebra, exactly as in $\S 2$. We set out, in an explicit form, some special properties of simple strata $[\mathfrak{A}, \ell, r, \beta]$ in $A$ for which the field extension $F[\beta] / F$ is tamely ramified. 
3.1. We start by recalling a fundamental property of tamely ramified simple strata.

Let $E / F$ be a finite, tamely ramified field extension, let $\beta \in E^{\times}$satisfy $E=F[\beta]$ and $v_{E}(\beta)<0$. Let $A(E)$ denote the split central simple $F$-algebra $\operatorname{End}_{F}(E)$ and let $\mathfrak{A}(E)$ denote the unique $E$-pure hereditary $\mathfrak{o}_{F}$-order in $A(E)$. Thus $\mathfrak{A}(E)$ is associated to the lattice chain $\left\{\mathfrak{p}_{E}^{k}: k \in \mathbb{Z}\right\}$ in the $F$-vector space $E$. Write $k_{F}(\beta)=k_{0}(\beta, \mathfrak{A}(E))$. We recall that $k_{F}(\beta) \leqslant v_{E}(\beta)$ if and only if $\beta$ is minimal over $F$.

Proposition. Suppose that $k_{F}(\beta)>v_{E}(\beta)$. There exists a simple stratum $\left[\mathfrak{A}(E),-v_{E}(\beta),-k_{F}(\beta), \gamma\right]$, equivalent to $\left[\mathfrak{A}(E),-v_{E}(\beta),-k_{F}(\beta), \beta\right]$ and with $\gamma \in$ E.

Proof. See 8.5 Lemma of [14].

Let $A$ be a central simple $F$-algebra of dimension $n^{2}$. Suppose that $E$ has been embedded in $A$ as an $F$-subalgebra. Let $\mathfrak{A}$ be an $E$-pure hereditary $\mathfrak{o}_{F}$-order in $A$.

Corollary. Let $\ell=-v_{\mathfrak{A}}(\beta)$ and $r=-k_{0}(\beta, \mathfrak{A})$. The stratum $[\mathfrak{A}, \ell, r, \gamma]$ is then simple and equivalent to $[\mathfrak{A}, \ell, r, \beta]$.

Proof. We have $\ell=-v_{E}(\beta) e\left(\mathfrak{A} \mid \mathfrak{o}_{F}\right) e(E \mid F)^{-1}$ and $r=-k(\beta) e\left(\mathfrak{A} \mid \mathfrak{o}_{F}\right) e(E \mid F)^{-1}$ (2.5). Applying these identities to $\gamma$, in place of $\beta$, the result follows.

3.2. We take a simple stratum $[\mathfrak{A}, \ell, 0, \beta]$ in $A$, such that $E=F[\beta] / F$ is tamely ramified. We define the orders $\mathfrak{H}(\beta, \mathfrak{A}), \mathfrak{J}(\beta, \mathfrak{A})$ and the simple character set $\mathfrak{C}\left(\mathfrak{A}, \beta, \psi_{F}\right)$ following the recipes in Propositions 3.42, 3.45 of [38]: these are strictly parallel to the treatment of the split case in [17]. It is convenient, in the present general discussion to impose the following simplifying assumption.

Hypothesis. There is a subfield $\widetilde{E} \supset E$ of $A$ such that $\mathfrak{A}$ is $\widetilde{E}$-pure, $\widetilde{E} / E$ is unramified and $[\widetilde{E}: F]=n$.

The field $\widetilde{E}$ is its own centralizer in $A$ and $\mathfrak{o}_{\widetilde{E}}$ is the unique $\mathfrak{o}_{\widetilde{E}}$-order in $\widetilde{E}$. It follows from $(2.3 .2)$ that $\mathfrak{A}$ is the unique $\widetilde{E}$-pure hereditary order in $A$. In particular, the hypothesis holds when the stratum $[\mathfrak{A}, \ell, 0, \beta]$ underlies a maximal simple type (as in 2.6 Definition). From the start of $\S 4$, we will always be in that situation. 
Let $K / F$ be a subfield of $\widetilde{E}$ : in particular, $K / F$ is tamely ramified. We let $A_{K}$ denote the $A$-centralizer of $K$ and $\mathfrak{A}_{K}=\mathfrak{A} \cap A_{K}$. Thus (2.3.2) $\mathfrak{A}_{K}$ is the unique $\widetilde{E}$-pure hereditary $\mathfrak{o}_{K}$-order in $A_{K}$. Let $G_{K}=A_{K}^{\times}$.

\section{Proposition.}

(1) The quadruple $\left[\mathfrak{A}_{K}, \ell, 0, \beta\right]$ is a simple stratum in $A_{K}$ and

$$
\begin{aligned}
\mathfrak{H}\left(\beta, \mathfrak{A}_{K}\right) & =\mathfrak{H}(\beta, \mathfrak{A}) \cap A_{K}, & \mathfrak{J}\left(\beta, \mathfrak{A}_{K}\right) & =\mathfrak{J}(\beta, \mathfrak{A}) \cap A_{K}, \\
H^{1}\left(\beta, \mathfrak{A}_{K}\right) & =H^{1}(\beta, \mathfrak{A}) \cap G_{K}, & J^{1}\left(\beta, \mathfrak{A}_{K}\right) & =J^{1}(\beta, \mathfrak{A}) \cap G_{K} .
\end{aligned}
$$

(2) Let $\psi_{K}=\psi_{F} \circ \operatorname{Tr}_{K / F}$ and let $\theta \in \mathfrak{C}\left(\mathfrak{A}, \beta, \psi_{F}\right)$. The restriction $\theta_{K}=\theta \mid$ $H^{1}\left(\beta, \mathfrak{A}_{K}\right)$ then lies in $\mathcal{C}\left(\mathfrak{A}_{K}, \beta, \psi_{K}\right)$.

Proof. Regarding the simplicity of $\left[\mathfrak{A}_{K}, \ell, 0, \beta\right]$ : this reduces to an assertion concerning the relation between $k_{F}(\beta)$ and $k_{K}(\beta)$. In other words, we need only consider the split case, where the result is given by [8] 2.4.

For the other assertions, we proceed by "induction along $\beta$ " in the manner familiar from [17]. Suppose first that $\beta$ is minimal over $F$. Thus either $\beta \in K$ or $\beta$ is minimal over $K$ (cf. [8] 2.9). Let $B$ denote the $A$-centralizer of $\beta$ and set $\mathfrak{B}=\mathfrak{A} \cap B$. We have $\mathfrak{H}(\beta, \mathfrak{A})=\mathfrak{B}+\mathfrak{P}^{[\ell / 2]+1}$, where $\mathfrak{P}=\operatorname{rad} \mathfrak{A}$. Define an integer $\ell^{\prime}$ by $\beta \mathfrak{A}_{K}=\mathfrak{P}_{K}^{-\ell^{\prime}}$, where $\mathfrak{P}_{K}=\operatorname{rad} \mathfrak{A}_{K}$. We then have $\mathfrak{P}^{[\ell / 2]+1} \cap A_{K} \supset \mathfrak{P}_{K}^{\left[\ell^{\prime} / 2\right]+1}$ whence $\mathfrak{H}(\beta, \mathfrak{A}) \cap A_{K} \supset \mathfrak{H}\left(\beta, \mathfrak{A}_{K}\right)$. Similarly for $\mathfrak{J}$ and, in multiplicative terms,

$$
H^{1}\left(\beta, \mathfrak{A}_{K}\right) \subset H^{1}(\beta, \mathfrak{A}) \cap G_{K}, \quad J^{1}\left(\beta, \mathfrak{A}_{K}\right) \subset J^{1}(\beta, \mathfrak{A}) \cap G_{K} .
$$

Take $\theta \in \mathfrak{C}\left(\mathfrak{A}, \beta, \psi_{F}\right)$ and consider the character $\vartheta=\theta \mid H^{1}(\beta, \mathfrak{A}) \cap G_{K}$. Certainly, $\vartheta$ is normalized by $J^{1}(\beta, \mathfrak{A}) \cap G_{K}$. Using the explicit form for simple characters, one sees that the restriction $\theta_{K}=\vartheta \mid H^{1}\left(\beta, \mathfrak{A}_{K}\right)$ lies in $\mathcal{C}\left(\mathfrak{A}_{K}, \beta, \psi_{K}\right)$. In particular, $\theta_{K}$ is intertwined by the subgroup $J^{1}(\beta, \mathfrak{A}) \cap G_{K}$ of $U_{\mathfrak{A}_{K}}^{1}$ and stable under conjugation by $J^{1}\left(\beta, \mathfrak{A}_{K}\right)$. It follows that $J^{1}(\beta, \mathfrak{A}) \cap G_{K}$ is contained in, and hence equal to, $J^{1}\left(\beta, \mathfrak{A}_{K}\right)$. The nondegeneracy property (2.5.4) implies that the character $\theta_{K}$ cannot be extended to a $J^{1}\left(\beta, \mathfrak{A}_{K}\right)$-stable character of a subgroup of $J^{1}\left(\beta, \mathfrak{A}_{K}\right)$ containing $H^{1}\left(\beta, \mathfrak{A}_{K}\right)$ strictly. All assertions follow in this case.

For the general step of the induction, let $r=-k_{0}(\beta, \mathfrak{A})<\ell$, and choose $\gamma \in E$ so that $[\mathfrak{A}, \ell, r, \gamma]$ is simple and equivalent to $[\mathfrak{A}, \ell, 0, \beta]$. Define the integer $r^{\prime}$ by 
$(\beta-\gamma) \mathfrak{A}_{K}=\mathfrak{P}_{K}^{-r^{\prime}}$. This gives $\mathfrak{H}(\beta, \mathfrak{A})=\mathfrak{B}+\mathfrak{H}^{[r / 2]+1}(\gamma, \mathfrak{A})$, where $\mathfrak{B}$ is the $\mathfrak{A}$ centralizer of $\beta$. We have $\mathfrak{P}^{[r / 2]+1} \cap A_{K}=\mathfrak{P}_{K}^{\left[r^{\prime} / 2\right]+1}$, so by inductive hypothesis we get $\mathfrak{H}(\beta, \mathfrak{A}) \cap A_{K} \supset \mathfrak{H}\left(\beta, \mathfrak{A}_{K}\right)$. Similarly for $\mathfrak{J}$. The argument concludes exactly as before.

We remark that some of the hypotheses can be weakened here, but we are not currently concerned with achieving generality.

3.3. By the term 1-pair we mean a pair $(E / F, \xi)$ in which $E / F$ is a finite, tamely ramified field extension and $\xi$ is a character of $U_{E}^{1}$. Two such pairs $\left(E_{i} / F, \xi_{i}\right)$, $i=1,2$, are said to be $F$-isomorphic if there is an $F$-isomorphism $\alpha: E_{1} \rightarrow E_{2}$ such that $\xi_{1}=\xi_{2} \circ \alpha$.

The 1-pair $(E / F, \xi)$ is called admissible if $\xi$ does not factor through $\mathrm{N}_{E / K}$, for any field $K$ satisfying $F \subset K \varsubsetneqq E$.

We return to the central simple algebra $A$ as above, and the simple stratum $[\mathfrak{A}, \ell, 0, \beta]$ in $A$, with $E=F[\beta] / F$ tamely ramified and satisfying 3.2 Hypothesis. Let $B$ denote the $A$-centralizer of $\beta$ and set $\mathfrak{B}=\mathfrak{A} \cap B$. Let $\theta \in \mathfrak{C}\left(\mathfrak{A}, \beta, \psi_{F}\right)$. The restriction of $\theta$ to $H^{1}(\beta, \mathfrak{A}) \cap B=U_{\mathfrak{B}}^{1}$ factors through $\operatorname{Nrd}_{B}$, [38] Proposition 3.47. That is, there exists a unique character $\xi_{\theta}$ of $U_{E}^{1}=\operatorname{Nrd}_{B}\left(U_{\mathfrak{B}}^{1}\right)$ such that $\theta \mid U_{\mathfrak{B}}^{1}=\xi_{\theta} \circ \operatorname{Nrd}_{B}$.

Proposition. The 1-pair $\left(E / F, \xi_{\theta}\right)$ is admissible: if $r_{0}=-k_{F}(\beta)$ then $\xi_{\theta} \mid U_{E}^{r_{0}}$ does not factor through $\mathrm{N}_{E / K}$ when $F \subset K \varsubsetneqq E$.

Proof. We first recall [11] A.1 Lemma:

Lemma. Let $E / F$ be a finite, tamely ramified field extension, and let $\psi$ be a character of $U_{E}^{i}$, for some $i \geqslant 1$. There exists a unique sub-extension $E^{\prime} / F$ of $E / F$, such that $\psi$ factors through $\mathrm{N}_{E / E^{\prime}}$, and which is minimal for this property.

We return to the proposition. If $E=F$, there is nothing to say, so we assume the contrary. We set $\ell_{0}=-v_{E}(\beta)$ and proceed by induction along $\beta$.

Assume first that $\beta$ is minimal over $F$. In this case, $r_{0}=\ell_{0}$. The character $\xi_{\theta}$ has level $\ell_{0}$, and $\xi_{\theta}(1+x)=\psi_{F}\left(\operatorname{Tr}_{E / F} \beta x\right), x \in \mathfrak{p}_{E}^{\ell_{0}}$. Therefore $\xi_{\theta} \mid U_{E}^{\ell_{0}}$ does not factor through any non-trivial norm map over $F$.

In general, we choose a simple stratum $\left[\mathfrak{A}(E), \ell_{0}, r_{0}, \gamma\right]$ equivalent to $\left[\mathfrak{A}(E), \ell_{0}\right.$, $\left.r_{0}, \beta\right]$, with $\gamma \in E$ (3.1). The element $\beta-\gamma \in E$ is then minimal over $F[\gamma]$. The 
character $\xi_{\theta}$ is of the form $\xi_{1} \xi_{2}$, for factors $\xi_{i}$ as follows. First, $\xi_{1}$ has level $r$ and $\xi_{1}(1+y)=\psi_{F}\left(\operatorname{Tr}_{E / F}(\beta-\gamma) y\right), y \in \mathfrak{p}_{E}^{r_{0}}$. As in the first step, $\xi_{1} \mid U_{E}^{r_{0}}$ does not factor through $\mathrm{N}_{E / K}$ if $F[\gamma] \subset K \varsubsetneqq E$. The other factor is of the form $\xi_{2}=\xi_{0} \circ \mathrm{N}_{E / F[\gamma]}$, for a character $\xi_{0}$ of $U_{F[\gamma]}^{1}$. By inductive hypothesis, $\xi_{2} \mid U_{E}^{r_{0}+1}$ does not factor through $\mathrm{N}_{E / K}$ if $F \subset K \varsubsetneqq F[\gamma]$. Consider the set of fields $L$, with $F \subset L \subset E$, such that $\xi_{2} \mid U_{E}^{r_{0}+1}$ factors through $\mathrm{N}_{E / L}$. By the lemma, this set has a unique minimal element, which is clearly $F[\gamma]$. We conclude that if $\xi_{\theta} \mid U_{E}^{r_{0}}$ factors through $\mathrm{N}_{E / K}$, then $K \supset F[\gamma]$. It follows that if $\xi_{\theta} \mid U_{E}^{r_{0}}$ factors through $\mathrm{N}_{E / K}$, then $K=E$, as required.

Comment. Théorème 3.53 of [38] gives a bijection

$$
\mathcal{C}\left(\mathfrak{A}(E), \beta, \psi_{F}\right) \cong \mathfrak{C}\left(\mathfrak{A}, \beta, \psi_{F}\right)
$$

that is canonical, for a fixed choice of $\beta$. Characters corresponding under this bijection give rise to the same admissible 1-pair. In the split case, elements of $\mathfrak{C}\left(\mathfrak{A}(E), \beta, \psi_{F}\right)$ give rise to isomorphic 1-pairs if and only if they are conjugate in $A(E)^{\times}=\operatorname{Aut}_{F}(E)$. This level of precision is not presently available in the general case.

\section{Attached pairs}

In this section, we give an approximate description of the elements of $\mathcal{A}_{m}^{\text {et }}(D)$, in terms of admissible pairs. Initially, this process involves arbitrary choices and has no particular invariance properties. The main results of later sections will show that it provides a canonical parametrization of the representations in $\mathcal{A}_{m}^{\text {et }}(D)$, generalizing that for $\mathcal{A}_{n}^{\text {et }}(F)$ in [11].

4.1. We recall a standard definition, $c f$. [11], [26].

Definition. Let $E / F$ be a finite, tamely ramified field extension and let $\xi$ be a character of $E^{\times}$. The pair $(E / F, \xi)$ is admissible if

(1) $\xi$ does not factor through $\mathrm{N}_{E / K}$, for any field $K$ such that $F \subset K \varsubsetneqq E$, and

(2) if $\xi \mid U_{E}^{1}$ does factor through $\mathrm{N}_{E / K}, F \subset K \subset E$, then $E / K$ is unramified.

We define $F$-isomorphism of admissible pairs in the same way as for 1-pairs (3.3). The degree of $(E / F, \xi)$ is $[E: F]$.

From 3.3 Lemma, we have: 
Lemma. Let $(E / F, \xi)$ be an admissible pair. There is a unique sub-extension $E_{0} / F$ of $E / F$ such that $\xi \mid U_{E}^{1}$ factors through $\mathrm{N}_{E / E_{0}}$, and which is minimal for this property.

The extension $E / E_{0}$ is, by definition, unramified. Putting the lemma another way, there is a unique admissible 1-pair $\left(E_{0} / F, \xi_{0}\right)$ with $E_{0} \subset E$ and $\xi \mid U_{E}^{1}=$ $\xi_{0} \circ \mathrm{N}_{E / E_{0}}$.

4.2. Let $\pi \in \mathcal{A}_{m}^{\text {et }}(D)$ have level zero. By 2.4 Theorem (1), $\pi$ contains a representation $\lambda$ of $\operatorname{GL}_{m}\left(\mathfrak{o}_{D}\right)$, inflated from an irreducible cuspidal representation $\tilde{\lambda}$ of $\mathrm{GL}_{m}\left(\mathbb{k}_{D}\right)$. The representation $\tilde{\lambda}$ is given, via the Green parametrization, by a character $\tilde{\xi}$ of $\boldsymbol{l}^{\times}$, where $\boldsymbol{l} / \mathbb{k}_{D}$ is a field extension of degree $m(c f$. (2.4.2)). Since (by definition) $\delta(\pi)=n$, the character $\tilde{\xi}$ is $\boldsymbol{l} / \mathbb{k}_{F}$-regular.

Let $E / F$ be unramified of degree $n$. We identify $\mathbb{k}_{E}$ with $\boldsymbol{l}$. We define a tamely ramified character $\xi$ of $E^{\times}$by deeming that $\xi \mid U_{E}$ shall be the inflation of $\tilde{\xi}$ and that $\xi \mid F^{\times}$shall be the central character of $\pi$.

Proposition. The pair $(E / F, \xi)$ is admissible. The process $\pi \mapsto(E / F, \xi)$ induces a canonical bijection between the set of $\pi \in \mathcal{A}_{m}^{\mathrm{et}}(D)$ of level zero and the set of $F$-isomorphism classes of admissible pairs $(E / F, \xi)$, of degree $n$, in which $\xi$ is tamely ramified.

Proof. The conjugates $\xi^{\sigma} \mid U_{E}, \sigma \in \operatorname{Gal}(E / F)$, are distinct, so $(E / F, \xi)$ is admissible. The second assertion restates 2.4 Theorem (3).

4.3. Let $\pi \in \mathcal{A}_{m}^{\text {et }}(D)$ be of positive level. Thus $\pi$ contains an extended maximal simple type $(\boldsymbol{J}, \Lambda)$, as in 2.6 Theorem. The extended type $(\boldsymbol{J}, \Lambda)$ is based on a simple stratum $[\mathfrak{A}, \ell, 0, \beta]$ in $A=\mathrm{M}_{m}(D)$ such that the field extension $E_{0}=$ $F[\beta] / F$ is tamely ramified (2.8 Lemma). The order $\mathfrak{A}$ is a maximal among $E_{0}$-pure hereditary orders in $A$. We let $B$ denote the $A$-centralizer of $E_{0}$ and $\mathfrak{B}=\mathfrak{A} \cap B$. Thus $\mathfrak{B}$ is a maximal $\mathfrak{o}_{E_{0}}$-order in $B$. As in the general discussion of 2.5 , there exists an unramified field extension $E / E_{0}$ inside $B$, such that $\mathfrak{B}$ is $E$-pure and $[E: F]=n$. In particular, 3.2 Hypothesis applies.

The restriction $\Lambda \mid H^{1}(\beta, \mathfrak{A})$ is a multiple of some $\theta \in \mathfrak{C}\left(\mathfrak{A}, \beta, \psi_{F}\right)$. There is a unique character $\xi_{0}$ of $U_{E_{0}}^{1}$ satisfying $\theta \mid U_{\mathfrak{B}}^{1}=\xi_{0} \circ \operatorname{Nrd}_{B}$ [38] Proposition 3.47. The 1-pair $\left(E_{0} / F, \xi_{0}\right)$ is admissible (3.3 Proposition). 
For the next step of the construction, we need to temporarily fix a prime element $\varpi_{F}$ of $F$.

Lemma 1. There exists a unique character $\xi_{\mathrm{w}}$ of $E^{\times}$such that

(a) $\xi_{\mathrm{w}} \mid U_{E}^{1}=\xi_{0} \circ \mathrm{N}_{E / E_{0}}$,

(b) $\xi_{\mathrm{w}}\left(\varpi_{F}\right)=1$, and

(c) $\xi_{\mathrm{w}}^{p^{a}}=1$, for some integer a.

The proof is immediate. For the next step, let $\eta$ be the unique irreducible representation of $J^{1}=J^{1}(\beta, \mathfrak{A})$ containing $\theta$. Abbreviate also $J=J(\beta, \mathfrak{A})$.

Lemma 2. There exists a unique irreducible representation $\Lambda_{\mathrm{w}}$ of $\boldsymbol{J}$ such that

(a) $\Lambda_{\mathrm{w}} \mid J$ is a wide extension of $\eta$,

(b) $\varpi_{F} \in \operatorname{Ker} \Lambda_{\mathrm{w}}$, and

(c) $\left(\operatorname{det} \Lambda_{\mathrm{w}}\right)^{p^{b}}=1$, for some integer $b$.

Proof. Conditions (a) and (c) determine $\Lambda_{\mathrm{w}} \mid J$ uniquely (cf. (2.5.5)), and are certainly achievable. Taken together with (b), they determine the restriction of $\Lambda$ to $F^{\times} J$. The hypothesis $\delta(\pi)=n$ implies that the group $\boldsymbol{J}$ is $E^{\times} J=E_{0}^{\times} J$, which contains $F^{\times} J$ with index $e\left(E_{0} \mid F\right)$. Since $p$ does not divide $e\left(E_{0} \mid F\right)$ and $\operatorname{dim} \Lambda_{\mathrm{w}}=\operatorname{dim} \eta$ is a power of $p$, the lemma follows.

As in the discussion of 2.6, we have a decomposition $\Lambda=\Lambda_{\mathrm{t}} \otimes \Lambda_{\mathrm{w}}$, for a uniquely determined representation $\Lambda_{\mathrm{t}}$ of $\boldsymbol{J}$, trivial on $J^{1}$. Writing $\boldsymbol{J}_{B}=\boldsymbol{J} \cap B$, we have $\boldsymbol{J}=\boldsymbol{J}_{B} J^{1}$ and the pair $\left(\boldsymbol{J}_{B}, \Lambda_{\mathrm{t}} \mid \boldsymbol{J}_{B}\right)$ is an extended maximal simple type in $B^{\times}$of level zero. Following 2.4 and 4.2 , it gives rise to an admissible pair $\left(E / E_{0}, \xi_{\mathrm{t}}\right)$ in which $\xi_{\mathrm{t}}$ is tamely ramified. We define $\xi=\xi_{\mathrm{t}} \cdot \xi_{\mathrm{w}}$.

Proposition. The pair $(E / F, \xi)$ is admissible of degree $n$, and satisfies $t(\pi)=$ $f(E \mid F)$. The definition of $\xi$ is independent of the choice of $\varpi_{F}$ in its construction.

The proof is immediate. An admissible pair obtained from $\pi$, in this manner, will be said to be attached to $\pi$.

Remark. Take $E, E_{0}$ and $\xi_{\mathrm{w}}$ as above. Let $\left(E / E_{0}, \vartheta\right)$ be an admissible pair with $\vartheta$ tamely ramified. The pair $\left(E / F, \vartheta \xi_{\mathrm{w}}\right)$ is then admissible and, following through the constructions of 2.6 , there exists a representation $\pi^{\prime} \in \mathcal{A}_{m}^{\text {et }}(D)$ to which this admissible pair is attached. 
4.4. Let $P_{n}(F)$ denote the set of $F$-isomorphism classes of admissible pairs over $F$, of degree $n$. In [11] 2.3, we produced a canonical bijection

$$
\begin{aligned}
P_{n}(F) & \longrightarrow \mathcal{A}_{n}^{\mathrm{et}}(F), \\
(E / F, \xi) & \longmapsto{ }_{F} \Pi_{\xi} .
\end{aligned}
$$

In this case, the construction of 4.3 is the inverse $\operatorname{map}{ }_{F} \Pi_{\xi} \mapsto(E / F, \xi)$. In the split case therefore, the construction is canonical, and yields a bijection $\mathcal{A}_{n}^{\text {et }}(F) \rightarrow P_{n}(F)$.

\section{Totally ramified representations}

In this section, we prove a special case of our main result. Throughout, $D$ denotes a central $F$-division algebra of dimension $d^{2}, d \geqslant 1$, and $A=\mathrm{M}_{m}(D)$, $G=\mathrm{GL}_{m}(D)$, where $m d=n$.

5.1. We establish the notation and hypotheses for the rest of the section. We take $\pi \in \mathcal{A}_{m}^{\text {et }}(D)$ and assume it is totally ramified, that is, $t(\pi)=1$. If we exclude the trivial case $n=1$, the representation $\pi$ then cannot be of level zero ( $c f .2 .4$ Theorem (4)).

We attach to $\pi$ an admissible pair $(E / F, \xi)$ as in 4.3 , using an extended maximal simple type $(\boldsymbol{J}, \Lambda)$ in $\pi$, which contains a simple character $\theta \in \mathfrak{C}\left(\mathfrak{A}, \beta, \psi_{F}\right)$, for some simple stratum $[\mathfrak{A}, \ell, 0, \beta]$ in $A$. In this case, the field $F[\beta]$ is $E$. By 4.3 Proposition, the extension $E / F$ is totally ramified of degree $n$ (and Hypothesis 3.2 is trivially verified). It follows that the hereditary order $\mathfrak{A}$ is minimal, and $\operatorname{rad} \mathfrak{A}=\varpi \mathfrak{A}$, for any prime element $\varpi$ of $E$. The group $\boldsymbol{J}$ is $E^{\times} J^{1}$, where $J^{1}=J^{1}(\beta, \mathfrak{A})$. Therefore $\Lambda \mid J^{1}$ is equivalent to the unique irreducible representation $\eta$ of $J^{1}$ which contains $\theta$.

5.2. We need a property of the character of the representation $\Lambda$.

Lemma 1. There is a constant $\epsilon_{A}= \pm 1$ such that

$$
\operatorname{tr} \Lambda(x)=\epsilon_{A} \xi(x),
$$

for every $x \in E$ with $v_{E}(x)$ relatively prime to $n$.

Proof. This is a standard application of the Glauberman correspondence, strictly parallel to the corresponding result 4.1 Lemma in [11]. 
As recalled in 2.5 , the space $\mathfrak{V}=\mathfrak{V}(\beta, \mathfrak{A})$ carries a nondegenerate alternating form $h_{\theta}$. The group $E^{\times}$acts on $\mathfrak{V}$ via its natural conjugation action on $J^{1}(\beta, \mathfrak{A})$, and this action fixes the form $h_{\theta}$ (as follows from $(2.5 .1)(3)$ ). The pair $\left(\mathfrak{V}, h_{\theta}\right)$ thus provides a symplectic representation over $\mathbb{F}_{p}$ of the cyclic group $\Psi=\Psi_{E / F}=$ $E^{\times} / F^{\times} U_{E}^{1}$. The symplectic sign $t_{\Psi}(\mathfrak{V}(\beta, \mathfrak{A}))$ is thus defined, as in [14] 3.4.

Lemma 2. The sign $\epsilon_{A}$ of (5.2.1) is given by

$$
\epsilon_{A}=t_{\Psi}(\mathfrak{V}(\beta, \mathfrak{A}))
$$

Proof. This is an instance of [6] (8.6.1).

5.3. We state the main result of the section. Let $\boldsymbol{j}_{A}: \mathcal{A}_{n}^{\mathrm{et}}(F) \rightarrow \mathcal{A}_{m}^{\mathrm{et}}(D)$ denote the bijection induced by the Jacquet-Langlands correspondence $\mathcal{A}_{n}^{\square}(F) \rightarrow$ $\mathcal{A}_{m}^{\square}(D)$. We use the notation ${ }_{F} \Pi_{\xi},(E / F, \xi) \in P_{n}(F)$, as in 4.4 .

Theorem. Let $\pi \in \mathcal{A}_{m}^{\mathrm{et}}(D)$ be totally ramified, and let $(E / F, \xi)$ be an admissible pair attached to $\pi$. If $\nu$ denotes the unramified character of $E^{\times}$given by

$$
\nu(x)=(-1)^{m(d-1) v_{E}(x)}, \quad x \in E^{\times},
$$

then

$$
\pi=j_{A}\left({ }_{F} \Pi_{\nu \xi}\right)
$$

Before proving the theorem, we note some consequences.

Corollary. Let $\pi \in \mathcal{A}_{m}^{\mathrm{et}}(D)$ be totally ramified.

(1) Any two admissible pairs attached to $\pi$ are F-isomorphic.

(2) The attachment process induces a canonical bijection between the set of totally ramified elements of $\mathcal{A}_{m}^{\mathrm{et}}(D)$ and the set of $F$-isomorphism classes of admissible pairs $(E / F, \xi)$ in which $E / F$ is totally ramified of degree $n$.

Proof. The assertions are valid in the case $\pi \in \mathcal{A}_{n}^{\text {et }}(F)$, by [11] 2.3 Theorem. The general case follows from the theorem.

5.4. We start the proof of the theorem. We use the Mackey formula (1.2.2) to follow the corresponding passage of [11]. 


\section{Proposition 1.}

(1) There exists $g \in G_{\text {reg }}^{\text {ell }}$ such that $\operatorname{gcd}\left(v_{F}\left(\operatorname{Nrd}_{A} g\right), n\right)=1$ and $\operatorname{tr} \pi(g) \neq 0$.

(2) Let $g \in G_{\mathrm{reg}}^{\mathrm{ell}}$ satisfy $\operatorname{gcd}\left(v_{F}\left(\operatorname{Nrd}_{A} g\right), n\right)=1$. If $\operatorname{tr} \pi(g) \neq 0$, then $g$ is $G$-conjugate to an element of $E^{\times}$.

Proof. Let $X_{n}$ denote the group of unramified characters $\chi$ of $F^{\times}$satisfying $\chi^{n}=1$. Since $\pi$ is totally ramified, the representations $\chi \pi, \chi \in X_{n}$, are distinct, whence the character functions $\operatorname{tr} \chi \pi: g \mapsto \chi\left(\operatorname{Nrd}_{A} g\right) \operatorname{tr} \pi(g)$ are linearly independent on $G_{\mathrm{reg}}^{\mathrm{ell}}(1.4 .5)$. The first assertion follows directly. The proof of the second is identical to that of part (1) of 4.1 Proposition of [11].

Let $\operatorname{Aut}(E \mid F)$ denote the group of $F$-automorphisms of the field $E$. Following the proof of part (2) of 4.1 Proposition of [11], we get:

Proposition 2. If $g \in E^{\times}$satisfies $\operatorname{gcd}\left(v_{E}(g), n\right)=1$ then

$$
\operatorname{tr} \pi(g)=\epsilon_{A} \sum_{\alpha \in \operatorname{Aut}(E \mid F)} \xi^{\alpha}(g)
$$

where $\epsilon_{A}= \pm 1$ is given by (5.2.2).

5.5. Define $\tau \in \mathcal{A}_{n}^{\text {et }}(F)$ by the condition $\pi=\boldsymbol{j}_{A}(\tau)$. There exists an admissible pair $(K / F, \zeta)$, uniquely determined up to $F$-isomorphism, such that $\tau={ }_{F} \Pi_{\zeta}$ [11] 2.3. We have $t(\tau)=t(\pi)=1$ (1.4.4), so $\tau$ is totally ramified. It follows that $K / F$ is totally ramified of degree $n$.

Applying 5.4 Proposition 1 to $\tau$, the character relation $\operatorname{tr} \pi=(-1)^{m(d-1)} \operatorname{tr} \tau$ implies that $K$ is $F$-isomorphic to $E$. We henceforth take $K=E$.

We apply 5.4 Proposition 2 to the representations $\pi, \tau$. If $\varpi$ is a prime element of $E$, we get

$$
\epsilon_{A} \sum_{\alpha \in \operatorname{Aut}(E \mid F)} \xi^{\alpha}\left(\varpi^{a} u\right)=(-1)^{m(d-1)} \epsilon_{M} \sum_{\alpha \in \operatorname{Aut}(E \mid F)} \zeta^{\alpha}\left(\varpi^{a} u\right),
$$

for $u \in U_{E}$, any integer $a$ relatively prime to $n$, and a constant $\epsilon_{M}= \pm 1$ obtained as follows. The representation $\tau$ contains a simple character $\theta_{M} \in \mathcal{C}\left(\mathfrak{M}, \gamma, \psi_{F}\right)$, for some simple stratum $\left[\mathfrak{M}, \ell_{M}, 0, \gamma\right]$ in $M=\mathrm{M}_{n}(F)$, with $F[\gamma]=E$ and $\theta_{M}$ | $U_{E}^{1}=\zeta \mid U_{E}^{1}$. The sign $\epsilon_{M}$ is then $t_{\Psi}(\mathfrak{V}(\gamma, \mathfrak{M}))$, in the notation of (5.2). 
We fix $a$ and view the two sides of (5.5.1) as functions of $u$. Since $E / F$ is totally ramified and $(E / F, \xi)$ is admissible, the characters $\xi^{\alpha} \mid U_{E}, \alpha \in \operatorname{Aut}(E \mid F)$, are distinct and hence linearly independent as functions on $U_{E}$. The same applies with $\zeta$ in place of $\xi$. We deduce that, for some $\alpha \in \operatorname{Aut}(E \mid F)$, we have $\xi \mid$ $U_{E}=\zeta^{\alpha} \mid U_{E}$. Replacing $(E / F, \zeta)$ by the isomorphic pair $\left(E / F, \zeta^{\alpha}\right)$ leaves $\tau$ unchanged, so we can assume that $\zeta$ agrees with $\xi$ on $U_{E}$.

Comparing coefficients of $\xi \mid U_{E}$ in (5.5.1), we obtain

$$
\zeta\left(\varpi^{a} u\right)=(-1)^{m(d-1)} \epsilon_{A} \epsilon_{M} \xi\left(\varpi^{a} u\right) .
$$

To complete the proof, it remains only to show that

$$
\epsilon_{A}=\epsilon_{M}
$$

5.6. We prove (5.5.2). As before, let $\mathfrak{A}, \mathfrak{M}$ be the unique $E$-pure hereditary orders in $A=\mathrm{M}_{m}(D), M=\mathrm{M}_{n}(F)$ respectively. Each of these has $F$-period $n=$ $e(E \mid F)$ and so is a minimal hereditary order. We have simple strata $[\mathfrak{A}, l, 0, \beta]$, $[\mathfrak{M}, l, 0, \gamma]$ in $A, M$, with $E=F[\beta]=F[\gamma]$. The $\epsilon$-invariants are given by

$$
\epsilon_{A}=t_{\Psi}(\mathfrak{V}(\mathfrak{A}, \beta)), \quad \epsilon_{M}=t_{\Psi}(\mathfrak{V}(\mathfrak{M}, \gamma))
$$

where $\Psi=\Psi_{E / F}$. This group is cyclic, generated by the image of $\varpi$. The desired relation (5.5.2) thus follows from:

Proposition. The symplectic $\mathbb{F}_{p} \Psi$-modules $\mathfrak{V}(\mathfrak{A}, \beta), \mathfrak{V}(\mathfrak{M}, \gamma)$ are isometric.

Proof. Indeed, it is enough to show that $\mathfrak{V}(\mathfrak{A}, \beta), \mathfrak{V}(\mathfrak{M}, \gamma)$ are isomorphic as $\mathbb{F}_{p} \Psi$-modules $[14] 3.3$.

Let $\mathfrak{P}=\operatorname{rad} \mathfrak{A}$. If $\varpi$ is a prime element of $E$, we have $\mathfrak{P}=\varpi \mathfrak{A}$, so all the quotients $\mathfrak{P}^{j} / \mathfrak{P}^{j+1}, j \in \mathbb{Z}$, are $\Psi$-isomorphic to $\mathfrak{A} / \mathfrak{P}$. Similarly for $\mathfrak{M}$ and $\mathfrak{P}_{\mathfrak{M}}=\operatorname{rad} \mathfrak{M}$.

Lemma. Each of the $\mathbb{F}_{p} \Psi$-modules $\mathfrak{A} / \mathfrak{P}, \mathfrak{M} / \mathfrak{P}_{\mathfrak{M}}$ is isomorphic to the regular representation $\mathbb{k}_{F} \Psi$.

Proof. In the case of $\mathfrak{M}$, the quotient $\mathfrak{M} / \mathfrak{P}_{\mathfrak{M}}$ is a sum of $n$ copies of $\mathbb{k}_{F}$, permuted cyclically by $\varpi$, with $\varpi^{n}$ acting trivially: it is therefore of the desired form. 
In the case of $\mathfrak{A}$, the quotient $\mathfrak{A} / \mathfrak{P}$ is the direct sum of $m$ copies of $\mathbb{k}_{D}$. The element $\varpi$ permutes these cyclically, and $\varpi^{m}$ acts on each factor $\mathbb{k}_{D}$ as a generator of $\operatorname{Gal}\left(\mathbb{k}_{D} / \mathbb{k}_{F}\right)$. By the Normal Basis Theorem, the $\mathbb{k}_{F} \Psi^{m}$-module $\mathbb{k}_{D}$ is free of rank one, so $\mathfrak{A} / \mathfrak{P}$ is $\mathbb{k}_{F} \Psi$-free of rank one, as required.

It is now convenient to choose the prime element $\varpi$, as we may, so that $\varpi^{n} \in F$. Thus, if $E^{\prime}$ is a field, $F \subset E^{\prime} \subset E$, then $E^{\prime}=F\left[\varpi^{\left[E: E^{\prime}\right]}\right]$.

Let $i \geqslant 0$ be an integer, and let $E_{i} / F$ be the least sub-extension of $E / F$ such that $\xi \mid U_{E}^{i+1}$ factors through $\mathrm{N}_{E / E_{i}}$ (cf. 3.3 Lemma). We recall that $i \geqslant 1$ is a jump of $\xi$ if $E_{i} \neq E_{i-1}$. Denote by $\mathfrak{W}_{M}$ the $\Psi$-module $\mathfrak{M} / \mathfrak{P}_{\mathfrak{M}}$, and let $\mathfrak{W}_{M}^{(i)}$ denote the submodule of $E_{i}^{\times}$fixed points: these are the same as the $\varpi^{n_{i}}$-fixed points, $n_{i}=\left[E: E_{i}\right]$.

Exactly as in [14] 8.5 (see also (2.5.4) above), the semisimple $\Psi$-module $\mathfrak{V}(\mathfrak{M}, \beta)$ is the direct sum of the modules $\mathfrak{W}_{M}^{(j)} / \mathfrak{W}_{M}^{(j+1)}$, where $2 j$ ranges over the even jumps of $\xi$. The same observation applies with $\mathfrak{A}, \mathfrak{W}=\mathfrak{A} / \mathfrak{P}$ in place of $\mathfrak{M}, \mathfrak{W}_{M}$. The proposition now follows from the lemma.

This completes the proof of 5.3 Theorem.

\section{Comparison and parametrization}

In this section, we extend the scope of 5.3 Corollary to obtain a canonical parametrization of the representations $\pi \in \mathcal{A}_{m}^{\text {et }}(D)$ in terms of admissible pairs. Simultaneously, we give the first step to describing the relation between this parametrization and the Jacquet-Langlands correspondence.

6.1. Let $\pi \in \mathcal{A}_{m}^{\text {et }}(D)$ have attached simple pair $(E / F, \xi)$. We use all the associated notations from the definition of $(E / F, \xi)$ in $\S 4$. In particular, $\pi$ contains an extended maximal simple type $(\boldsymbol{J}, \Lambda)$. If $\pi$ has positive level, $(\boldsymbol{J}, \Lambda)$ is based on a simple stratum $[\mathfrak{A}, \ell, 0, \beta]$ in $A$, satisfying Hypothesis 3.2. The representation $\Lambda$ contains a simple character $\theta \in \mathcal{C}\left(\mathfrak{A}, \beta, \psi_{F}\right)$. We set $E_{0}=F[\beta]$ and let $B$ denote the $A$-centralizer of $E_{0}$. We also set $\mathfrak{B}=\mathfrak{A} \cap B, \mathfrak{Q}=\mathfrak{P} \cap B=\operatorname{rad} \mathfrak{B}$.

We prove:

\section{Parametrization Theorem.}

(1) Let $\pi \in \mathcal{A}_{m}^{\text {et }}(D)$ have attached simple pair $(E / F, \xi)$. If $\left(E^{\prime} / F, \xi^{\prime}\right)$ is another simple pair attached to $\pi$, then $\left(E^{\prime} / F, \xi^{\prime}\right)$ is $F$-isomorphic to 
$(E / F, \xi)$

(2) Let $(E / F, \xi)$ be an admissible pair of degree $n$. There exists a unique element ${ }_{D} \Pi_{\xi} \in \mathcal{A}_{m}^{\mathrm{et}}(D)$ with attached admissible pair $F$-isomorphic to $(E / F, \xi)$. The map

$$
\begin{aligned}
P_{n}(F) & \longrightarrow \mathcal{A}_{m}^{\mathrm{et}}(D), \\
(E / F, \xi) & \longmapsto{ }_{D} \Pi_{\xi},
\end{aligned}
$$

is a bijection.

Remark. For representations $\pi$ of level zero and admissible pairs $(E / F, \xi)$ with $\xi$ tamely ramified, the theorem reduces to 4.2 Proposition.

The simple stratum $[\mathfrak{A}, \ell, 0, \beta]$ gives rise to the group $\mathfrak{V}=\mathfrak{V}(\beta, \mathfrak{A})$, which furnishes a symplectic representation of the finite abelian group $\Psi=E^{\times} / U_{E}^{1} F^{\times}$ over $\mathbb{F}_{p}$. In particular, it gives a symplectic representation of the cyclic group $\boldsymbol{\mu}_{E}$, so the fine symplectic signs $t_{\boldsymbol{\mu}_{E}}^{k}(\mathfrak{V}), k=0,1$, are defined as in [14] $\S 3$. We recall that $t_{\boldsymbol{\mu}_{E}}^{0}(\mathfrak{V})= \pm 1$ is a constant, while $t_{\boldsymbol{\mu}_{E}}^{1}(\mathfrak{V})$ is a character of $\boldsymbol{\mu}_{E}$ with values in $\{ \pm 1\}$.

The symplectic representation of $\Psi$ over $\mathbb{F}_{p}$ afforded by $\mathfrak{V}(\beta, \mathfrak{A})$ depends, up to isometry, only on the algebra $A$ and the restriction of $\xi$ to $U_{E}^{1}$. Indeed, as in [14], it depends only on the sequence of "jump fields" $E_{i} / F$ defined by this character. We therefore use a notation parallel to that of [14],

$$
{ }_{A} \epsilon_{(E / F, \xi)}^{k}\left(\boldsymbol{\mu}_{E}\right)=t_{\boldsymbol{\mu}_{E}}^{k}(\mathfrak{V}), \quad k=0,1,
$$

or, more commonly, the abbreviated version

$$
\epsilon_{A}^{k}\left(\boldsymbol{\mu}_{E}\right)={ }_{A} \epsilon_{(E / F, \xi)}^{k}\left(\boldsymbol{\mu}_{E}\right) .
$$

We remark that, in the case where $\pi$ has level zero, the $\epsilon$-invariants are all trivial.

We shall also prove:

First Comparison Theorem. Let $(E / F, \xi)$ be an admissible pair of degree $n$. There exists a unique tamely ramified character $\nu={ }_{D} \nu_{\xi}$ of $E^{\times}$with the following properties.

(1) The restriction of $\nu$ to $\boldsymbol{\mu}_{E}$ is the character

$$
\epsilon_{A}^{1}\left(\boldsymbol{\mu}_{E}\right) \epsilon_{M}^{1}\left(\boldsymbol{\mu}_{E}\right)={ }_{A} \epsilon_{(E / F, \xi)}^{1}\left(\boldsymbol{\mu}_{E}\right) \cdot{ }_{M} \epsilon_{(E / F, \xi)}^{1}\left(\boldsymbol{\mu}_{E}\right) .
$$


(2) The pair $(E / F, \nu \xi)$ is admissible and

$$
{ }_{D} \Pi_{\xi}=\boldsymbol{j}_{A}\left({ }_{F} \Pi_{\nu \xi}\right)
$$

where $\boldsymbol{j}_{A}: \mathcal{A}_{n}^{\mathrm{et}}(F) \rightarrow \mathcal{A}_{m}^{\mathrm{et}}(D)$ is the Jacquet-Langlands correspondence.

The proofs of the two theorems are entwined, and occupy the rest of the section.

6.2. We take $\pi \in \mathcal{A}_{m}^{\mathrm{et}}(D)$ with attached simple pair $(E / F, \xi)$, and use the notation of 6.1. Until declared otherwise (at the end of 6.8), we assume that $\pi$ does not have level zero or, equivalently, that $\xi$ is not tamely ramified.

As in 2.1, the $E_{0}$-algebra $B$ takes the form $B \cong \mathrm{M}_{l}(C)$, for an integer $l$ and a central $E_{0}$-division algebra $C$ of dimension $c^{2}, l c=n /\left[E_{0}: F\right]$.

Let $K / F$ be the maximal unramified sub-extension of $E / F$. Set

$$
\Sigma=\operatorname{Gal}(K / F), \quad \Sigma_{0}=\operatorname{Gal}\left(E / E_{0}\right) .
$$

If $K_{0}=K \cap E_{0}$, restriction of operators identifies $\Sigma_{0}$ with $\operatorname{Gal}\left(K / K_{0}\right)$.

Let $A_{K}$ denote the $A$-centralizer of $K, G_{K}=A_{K}^{\times}$. Thus $A_{K} \cong \mathrm{M}_{m_{K}}\left(D_{K}\right)$, for a central $K$-division algebra $D_{K}$ of $K$-dimension $d_{K}^{2}$, say, and $m_{K} d_{K}=n /[K: F]$.

Set $\mathfrak{A}_{K}=\mathfrak{A} \cap A_{K}$. Thus $\mathfrak{A}_{K}$ is a hereditary $\mathfrak{o}_{K}$-order in $A_{K}$. It is $E$-pure, and is the unique $E$-pure hereditary order in $A_{K}$ (cf. 2.3). From 3.2 Proposition, we have:

(1) The stratum $\left[\mathfrak{A}_{K}, \ell, 0, \beta\right]$ is simple.

(2) We have the relations

$$
H^{1}\left(\beta, \mathfrak{A}_{K}\right)=H^{1}(\beta, \mathfrak{A}) \cap G_{K}, \quad J^{1}\left(\beta, \mathfrak{A}_{K}\right)=J^{1}(\beta, \mathfrak{A}) \cap G_{K} .
$$

(3) The restriction $\theta \mid H^{1}\left(\beta, \mathfrak{A}_{K}\right)=\theta_{K}$ lies in $\mathfrak{C}\left(\mathfrak{A}_{K}, \beta, \psi_{K}\right)$, where $\psi_{K}=$ $\psi_{F} \circ \operatorname{Tr}_{K / F}$.

The discussion in 3.2 likewise gives the relation

$$
H^{1}(\beta, \mathfrak{A}) \cap E=U_{E}^{1}, \quad \text { and } \quad \theta\left|U_{E}^{1}=\xi\right| U_{E}^{1} .
$$

The same holds with $\left(\mathfrak{A}_{K}, \theta_{K}\right)$ replacing $(\mathfrak{A}, \theta)$. 
6.3. We fix a root of unity $\zeta \in \boldsymbol{\mu}_{K}=\boldsymbol{\mu}_{E}$ such that $K=F[\zeta]$. We let $u$ range over the set ${ }_{\mathrm{u}}\left(G_{K}\right)_{\mathrm{reg}}^{\text {ell }}$ of pro-unipotent, elliptic regular elements of $G_{K}$. (Regularity and ellipticity are measured relative to the base field $K$ in this set.) The element $h=\zeta u$ then lies in $G_{\text {reg }}^{\text {ell }}$, so we may use (1.2.2) to evaluate the character of $\pi$ at $h$,

$$
\operatorname{tr} \pi(h)=\sum_{x \in G / J} \operatorname{tr} \Lambda\left(x^{-1} h x\right) .
$$

A coset $x \boldsymbol{J}$ can only contribute to the sum if $x^{-1} h x \in \boldsymbol{J}$ or, equivalently, $x^{-1} h x \in$ $J^{0}$.

Lemma. Let $x \in G$ and suppose $x^{-1} h x \in J^{0}$. There then exists $y \in N_{G}\left(K^{\times}\right)$ such that $x \boldsymbol{J}=y \boldsymbol{J}$. For any such $y$, we have $y^{-1} u y \in J^{1}$ (and hence $y^{-1} u y \in$ $\left.J^{1} \cap G_{K}\right)$.

Proof. This is identical to the proof of 5.4 Lemma 1 in [14]: as noted there, the argument applies to pro-unipotent, elliptic regular elements $u$ of $G_{K}$.

The sum in (6.3.1) is therefore effectively taken over the space $N_{G}\left(K^{\times}\right) \boldsymbol{J} / \boldsymbol{J}$, which we now describe more conveniently.

Let $\Sigma_{\mathrm{t}}$ denote the unique subgroup of $\Sigma_{0}=\operatorname{Gal}\left(E / E_{0}\right)$ of index $c$. The residue field $\mathbb{k}_{E}$ is a maximal subfield of the algebra $\mathfrak{B} / \mathfrak{Q} \cong \mathrm{M}_{l}\left(\mathbb{k}_{C}\right)$. The group $\Sigma$ is canonically identified with $\operatorname{Gal}\left(\mathbb{k}_{E} / \mathbb{k}_{F}\right)$ via its actions on roots of unity. The subgroups $\Sigma_{0}, \Sigma_{\mathrm{t}}$ are then $\mathrm{Gal}\left(\mathbb{k}_{E} / \mathbb{k}_{E_{0}}\right)$ and $\mathrm{Gal}\left(\mathbb{k}_{E} / \mathbb{k}_{C}\right)$ respectively.

For each $\sigma \in \Sigma$, we choose $t_{\sigma} \in N_{G}\left(K^{\times}\right)$with image $\sigma$ under the canonical isomorphism $N_{G}\left(K^{\times}\right) / G_{K} \cong \Sigma$. Observe that the subgroup $\Sigma_{\mathrm{t}}$ of $\Sigma$ is the image of $\boldsymbol{J} \cap N_{G}\left(K^{\times}\right)$under this map.

Proposition. We have

$$
N_{G}\left(K^{\times}\right) \boldsymbol{J}=\bigcup_{\sigma \in \Sigma / \Sigma_{\mathrm{t}}} G_{K} t_{\sigma} \boldsymbol{J}
$$

the union being disjoint, and hence a bijection

$$
\begin{aligned}
G_{K} \backslash N_{G}\left(K^{\times}\right) \boldsymbol{J} / \boldsymbol{J} & \longrightarrow \Sigma / \Sigma_{\mathrm{t}}, \\
G_{K} t_{\sigma} \boldsymbol{J} & \longmapsto \sigma \Sigma_{\mathrm{t}} .
\end{aligned}
$$


Proof. The space $N_{G}\left(K^{\times}\right) \boldsymbol{J}$ surely decomposes as the union of double cosets $G_{K} t_{\sigma} \boldsymbol{J}, \sigma \in \Sigma$. We have to show that, if $\sigma, \tau \in \Sigma$, then $G_{K} t_{\sigma} \boldsymbol{J}=G_{K} t_{\tau} \boldsymbol{J}$ if and only if $\sigma^{-1} \tau \in \Sigma_{\mathrm{t}}$.

The elements $t_{\sigma}, t_{\tau}$ normalize $G_{K}$, whence $G_{K} t_{\sigma} \boldsymbol{J}=G_{K} t_{\tau} \boldsymbol{J}$ if and only if $t_{\sigma}^{-1} t_{\tau} \in G_{K} \boldsymbol{J}$. This, in turn, is equivalent to the automorphism $\alpha \mapsto \alpha^{\sigma^{-1} \tau}$ being realized as conjugation by an element of $\boldsymbol{J}$. That is, $\sigma^{-1} \tau \in \Sigma_{\mathrm{t}}$, as required.

The formula (6.3.1) has become

$$
\operatorname{tr} \pi(\zeta u)=\sum_{\alpha \in \Sigma / \Sigma_{\mathrm{t}}} \sum_{y \in G_{K} / \boldsymbol{J}_{K}} \operatorname{tr} \Lambda\left(y^{-1} \zeta^{\alpha} u^{\alpha} y\right)
$$

This expression is valid for any $K / F$-regular root of unity $\zeta \in \boldsymbol{\mu}_{K}$ and any $u \in{ }_{\mathrm{u}}\left(G_{K}\right)_{\text {reg }}^{\text {ell }}$. Only finitely many of the terms $\operatorname{tr} \Lambda\left(y^{-1} \zeta^{\alpha} u^{\alpha} y\right)$ are non-zero (1.2 Lemma).

6.4. Let $\eta_{K}$ be the unique irreducible representation of $J_{K}^{1}=J^{1}\left(\beta, \mathfrak{A}_{K}\right)$ which contains $\theta_{K}$. The field extension $E=K[\beta] / K$ is totally ramified and maximal in $A_{K}$. It follows that the group $J_{K}=J\left(\beta, \mathfrak{A}_{K}\right)=J \cap G_{K}$ is $U_{E} J_{K}^{1}=\boldsymbol{\mu}_{E} J_{K}^{1}$.

We take a prime element $\varpi_{F}$ of $F$, and use it to define a factorization $\xi=\xi_{\mathrm{t}} \cdot \xi_{\mathrm{w}}$ of $\xi$, reversing the procedure of 4.3. Explicitly, $\xi_{\mathrm{w}}$ agrees with $\xi$ on $U_{E}^{1}$, it has finite $p$-power order, and $\xi_{\mathrm{w}}\left(\varpi_{F}\right)=1$. The character $\xi_{\mathrm{t}}$ is $\xi_{\mathrm{w}}^{-1} \xi$.

Since $E=E_{0} K$, the pair $\left(E / K, \xi_{\mathrm{w}}\right)$ is admissible and totally ramified. The character $\xi_{\mathrm{t}}$ is tamely ramified, and the pair $\left(E / E_{0}, \xi_{\mathrm{t}}\right)$ is admissible.

We extend $\eta_{K}$ to a representation ${ }_{K} \lambda_{\mathrm{w}}$ of $J_{K}=\boldsymbol{\mu}_{E} J_{K}^{1}$ by deeming that ${ }_{K} \lambda_{\mathrm{w}} \mid \boldsymbol{\mu}_{E}$ be trivial. The pair $\left(J_{K},{ }_{K} \lambda_{\mathrm{w}}\right)$ is then a maximal simple type in $G_{K}$. The group $\boldsymbol{J}\left({ }_{K} \lambda_{\mathrm{w}}\right)$ is $\boldsymbol{J}_{K}=\boldsymbol{J} \cap G_{K}=E^{\times} J_{K}^{1}$. We extend ${ }_{K} \lambda_{\mathrm{w}}$ to a representation ${ }_{K} \Lambda_{\mathrm{w}}$ of $\boldsymbol{J}_{K}$ so that $\varpi_{F} \in \operatorname{Ker}_{K} \Lambda_{\mathrm{w}}$ and $\operatorname{det}_{K} \Lambda_{\mathrm{w}}$ has finite, $p$ power order. Thus $\left(\boldsymbol{J}_{K},{ }_{K} \Lambda_{\mathrm{w}}\right)$ is an extended maximal simple type in $G_{K}$, with the property $\boldsymbol{\mu}_{K} \subset \operatorname{Ker}_{K} \Lambda_{\mathrm{w}}$. We define

$$
\rho_{\mathrm{w}}=c-\operatorname{Ind}_{\boldsymbol{J}_{K}}^{G_{K}} \Lambda_{\mathrm{w}} .
$$

This is an irreducible, totally ramified, essentially tame, cuspidal representation of $G_{K}$, with maximal parametric degree

$$
\delta\left(\rho_{\mathrm{w}}\right)=[E: K]=\left(\operatorname{dim}_{K} A_{K}\right)^{1 / 2},
$$


and attached admissible pair $\left(E / K, \xi_{\mathrm{w}}\right)$.

In the formula (6.3.2), $\zeta^{\alpha}$ commutes with $y$ and, by 6.3 Lemma, the element $y^{-1} u^{\alpha} y$ lies in $J_{K}^{1}$. We decompose $\Lambda=\Lambda_{\mathrm{t}} \otimes \Lambda_{\mathrm{w}}$, as in the definition of 4.3. Since $\Lambda_{\mathrm{t}}$ is trivial on $J^{1}$, we are reduced to

$$
\operatorname{tr} \pi(\zeta u)=\sum_{\alpha \in \Sigma / \Sigma_{\mathrm{t}}} \operatorname{tr} \Lambda_{\mathrm{t}}\left(\zeta^{\alpha}\right) \sum_{y \in G_{K} / J_{K}} \operatorname{tr} \Lambda_{\mathrm{w}}\left(\zeta^{\alpha} y^{-1} u^{\alpha} y\right) .
$$

The definitions of $\Lambda_{\mathrm{w}},{ }_{K} \Lambda_{\mathrm{w}}$ yield

$$
\begin{aligned}
\operatorname{tr} \Lambda_{\mathrm{w}}\left(\zeta^{\alpha} y^{-1} u^{\alpha} y\right) & =\epsilon_{A} \operatorname{tr}_{K} \Lambda_{\mathrm{w}}\left(y^{-1} u^{\alpha} y\right) \\
& =\epsilon_{A} \operatorname{tr} \eta_{K}\left(y^{-1} u^{\alpha} y\right),
\end{aligned}
$$

where

$$
\epsilon_{A}=t_{\langle\zeta\rangle}(\mathfrak{V})=t_{\boldsymbol{\mu}_{E}}^{0}(\mathfrak{V}) t_{\boldsymbol{\mu}_{E}}^{1}\left(\mathfrak{V} ; \zeta^{\alpha}\right)=t_{\boldsymbol{\mu}_{E}}^{0}(\mathfrak{V}) t_{\boldsymbol{\mu}_{E}}^{1}(\mathfrak{V} ; \zeta),
$$

and $\mathfrak{V}=\mathfrak{V}(\beta, \mathfrak{A})(c f .[14](3.4 .3),(5.6 .2))$. The inner sum in (6.4.1) is therefore reduced to

$$
\sum_{y \in G_{K} / J_{K}} \operatorname{tr} \Lambda_{\mathrm{w}}\left(\zeta^{\alpha} y^{-1} u^{\alpha} y\right)=\epsilon_{A} \operatorname{tr} \rho_{\mathrm{w}}^{\alpha^{-1}}(u) .
$$

Overall, we have

$$
\operatorname{tr} \pi(\zeta u)=\epsilon_{A} \sum_{\alpha \in \Sigma / \Sigma_{\mathrm{t}}} \operatorname{tr} \Lambda_{\mathrm{t}}\left(\zeta^{\alpha}\right) \operatorname{tr} \rho_{\mathrm{w}}^{\alpha^{-1}}(u) .
$$

This relation is valid for all $\Sigma$-regular roots of unity $\zeta \in \boldsymbol{\mu}_{K}=\boldsymbol{\mu}_{E}$ and all pro-unipotent, elliptic regular elements $u$ of $G_{K}$.

6.5. We examine the representations $\rho_{\mathrm{w}}^{\alpha}=\rho_{\mathrm{w}} \circ \alpha, \alpha \in \Sigma$, of the group $G_{K}$.

Lemma. Let $\alpha \in \Sigma$. The following conditions are equivalent.

(1) $\rho_{\mathrm{w}}^{\alpha} \cong \rho_{\mathrm{w}}$

(2) there is an unramified character $\chi$ of $K^{\times}$such that $\rho_{\mathrm{w}}^{\alpha} \cong \chi \rho_{\mathrm{w}}$;

(3) $\alpha \in \Sigma_{0}$.

Proof. The representation $\rho_{\mathrm{w}}$ is, by construction, attached to the admissible pair $\left(E / K, \xi_{\mathrm{w}}\right)$ and $E / K$ is totally ramified. If $\alpha \in \Sigma_{0}$, then $\rho_{\mathrm{w}}^{\alpha}$ is attached to the admissible pair $\left(E / K, \xi_{\mathrm{w}}^{\alpha}\right)$, while $\xi_{\mathrm{w}}^{\alpha}=\xi_{\mathrm{w}}$. By 5.3 Corollary, we have $\rho_{\mathrm{w}}^{\alpha} \cong \rho_{\mathrm{w}}$. 
Thus (3) $\Rightarrow(1)$ and surely (1) $\Rightarrow(2)$. We assume, therefore, that (2) holds. Let $\left(E^{\prime} / K, \xi^{\prime}\right)$ be the admissible pair attached to $\rho_{\mathrm{w}}^{\alpha}$ : since $\rho_{\mathrm{w}}^{\alpha}$ is totally ramified, the pair $\left(E^{\prime} / K, \xi^{\prime}\right)$ is uniquely determined up to $K$-isomorphism (5.3 Corollary). It satisfies the following condition: there is a field isomorphism $\alpha^{\prime}: E \rightarrow E^{\prime}$, such that $\alpha^{\prime} \mid K=\alpha$, which carries $\xi_{\mathrm{w}}$ to $\xi^{\prime}$. However, the hypothesis $\chi \rho_{\mathrm{w}} \cong \rho_{\mathrm{w}}^{\alpha}$ implies that the admissible 1-pairs $\left(E / K, \xi_{\mathrm{w}} \mid U_{E}^{1}\right),\left(E^{\prime} / K, \xi^{\prime} \mid U_{E}^{1}\right)$ are $K$-isomorphic. If this isomorphism is realized by a $K$-isomorphism $\alpha^{\prime \prime}: E \rightarrow E^{\prime}$, then $\alpha^{\prime \prime-1} \alpha^{\prime}$ is an automorphism of $E$, extending the automorphism $\alpha$ of $K$, which fixes $\xi_{\mathrm{w}}\left|U_{E}^{1}=\xi\right| U_{E}^{1}$. It follows that $\alpha^{\prime \prime}{ }^{-1} \alpha^{\prime} \in \Sigma_{0}$. We may therefore take $E^{\prime}=E$ and the same argument gives $\alpha \in \Sigma_{0}$, as required.

6.6. In order to apply 6.5 Lemma, we need a general result on linear independence of cuspidal characters.

Linear Independence Lemma. Let $\pi_{1}, \pi_{2}, \ldots, \pi_{r}$ be irreducible, totally ramified, cuspidal representations of $G$, all of parametric degree $n$. Suppose

(a) the central characters of the $\pi_{i}$ all agree on some prime element of $F$ and on $\boldsymbol{\mu}_{F}$;

(b) the representation $\pi_{i}$ is not equivalent to an unramified twist of $\pi_{j}$ whenever $i \neq j$.

The characters $\operatorname{tr} \pi_{i}, 1 \leqslant i \leqslant r$, then form a linearly independent set of functions on ${ }_{\mathrm{u}} G_{\mathrm{reg}}^{\mathrm{ell}}$.

Proof. The Jacquet-Langlands correspondence commutes with twisting by characters of $F^{\times}$(1.4.3), and preserves central characters (1.4.2). It also preserves parametric degree 2.8 Corollary 1. It is therefore enough to treat the case $G=\mathrm{GL}_{1}(D)$, for some central $F$-division algebra $D$ of dimension $n^{2}$.

We use the classification theory of [4] for representations of $G$. Since $\pi_{j}$ is totally ramified, there is a simple stratum $\left[\mathfrak{o}_{D}, l_{j}, 0, \beta_{j}\right]$ in $D$, and a simple character $\theta_{j} \in \mathcal{C}\left(\mathfrak{o}_{D}, \beta_{j}, \psi\right)$ which occurs in $\pi_{j}$. Since $\pi_{j}$ is totally ramified of parametric degree $n$, the field extension $F\left[\beta_{j}\right] / F$ is totally ramified of degree $n$. The hypotheses on the representations $\pi_{j}$ are equivalent to $\theta_{i}$ not intertwining with $\theta_{j}$ when $i \neq j$. The proof now follows the same course as that of 5.7 Lemma (3) in [14]. 
6.7. In (6.4.2), the definition of $\Lambda_{\mathrm{t}}$ gives

$$
\operatorname{tr} \Lambda_{\mathrm{t}}\left(\zeta^{\alpha}\right)=(-1)^{l-1} \sum_{\gamma \in \Sigma_{\mathrm{t}}} \xi_{\mathrm{t}}\left(\zeta^{\alpha \gamma}\right)
$$

where, we recall, $B \cong \mathrm{M}_{l}(C)$ and $U_{\mathfrak{B}} / U_{\mathfrak{B}}^{1} \cong \mathrm{GL}_{l}\left(\mathbb{k}_{C}\right)$. We therefore re-write (6.4.2) in the form

$$
\operatorname{tr} \pi(\zeta u)=(-1)^{l-1} \epsilon_{A} \sum_{\alpha \in \Sigma / \Sigma_{0}} \operatorname{tr} \rho_{\mathrm{w}}^{\alpha}(u) \sum_{\gamma \in \Sigma_{0}} \xi_{\mathrm{t}}\left(\zeta^{\alpha \gamma}\right) .
$$

We turn to the corresponding situation in $\mathrm{GL}_{n}(F)$. We take $\tau \in \mathcal{A}_{n}^{\text {et }}(F)$ to satisfy $\boldsymbol{j} \tau=\pi$. The representation $\tau$ is of the form ${ }_{F} \Pi_{\phi}$, for an admissible pair $\left(E^{\prime} / F, \phi\right)$, uniquely determined up to isomorphism. The relation $t(\tau)=t(\pi)$ implies $f(E \mid F)=f\left(E^{\prime} \mid F\right)$, so we may identify the maximal unramified subextension of $E^{\prime} / F$ with $K / F$ and continue to use the notation $\Sigma=\operatorname{Gal}(K / F)$ on either side. Let $E_{0}^{\prime} / F$ be the minimal sub-extension of $E^{\prime} / F$ such that $\phi \mid U_{E^{\prime}}^{1}$ factors through $\mathrm{N}_{E^{\prime} / E_{0}^{\prime}}$ and set $\Sigma_{0}^{\prime}=\operatorname{Gal}\left(E^{\prime} / E_{0}^{\prime}\right)$. We use the same prime element $\varpi_{F}$ of $F$ to achieve a factorization $\phi=\phi_{\mathrm{t}} \cdot \phi_{\mathrm{w}}$, as was applied to $\xi$ in 6.4 .

Applying (6.7.1) to this case, we get an expression

$$
\operatorname{tr} \tau(\zeta v)=(-1)^{k-1} \epsilon_{M} \sum_{\alpha \in \Sigma / \Sigma_{0}^{\prime}} \operatorname{tr} \sigma_{\mathrm{w}}^{\alpha}(v) \sum_{\gamma \in \Sigma_{0}^{\prime}} \phi_{\mathrm{t}}\left(\zeta^{\alpha \gamma}\right) .
$$

Here, $k=\left[E^{\prime}: E_{0}^{\prime}\right]$. The simple stratum in $M=\mathrm{M}_{n}(F)$ attached to $\left(E^{\prime} / F, \phi\right)$ is $\left[\mathfrak{M}, \ell_{M}, 0, \delta\right]$, say, and

$$
\epsilon_{M}=t_{\langle\zeta\rangle}(\mathfrak{V}(\delta, \mathfrak{M}))=\epsilon_{M}^{0}\left(\boldsymbol{\mu}_{E}\right) \epsilon_{M}^{1}\left(\boldsymbol{\mu}_{E} ; \zeta\right) .
$$

The representation $\sigma_{\mathrm{w}}$ is ${ }_{K} \Pi_{\phi_{\mathrm{w}}}$. The equation (6.7.2) is valid for all $K / F$-regular roots of unity $\zeta \in \boldsymbol{\mu}_{K}$ and all pro-unipotent, elliptic regular elements $v$ of the $\mathrm{GL}_{n}(F)$-centralizer of $K^{\times}$.

We now use the lemmas of $6.5,6.6$ to conclude that, for a suitable choice of $\left(E^{\prime} / F, \phi\right)$, we get

$$
\rho_{\mathrm{w}}=\boldsymbol{j}_{K}\left(\chi \sigma_{\mathrm{w}}\right)
$$

for an unramified character $\chi$ of $K^{\times}$, where $\boldsymbol{j}_{K}$ is a Jacquet-Langlands correspondence with base field $K$. Therefore (5.3 Corollary) $\left(E^{\prime} / K, \phi_{\mathrm{w}}\right)$ is $K$-isomorphic 
to an unramified twist of $\left(E / K, \xi_{\mathrm{w}}\right)$. We may henceforth take $E^{\prime}=E, E_{0}^{\prime}=E_{0}$, and $\Sigma=\Sigma^{\prime}$. In particular, $k=l c, c^{2}=\operatorname{dim}_{E_{0}} C$.

We use 6.6 Lemma again to compare coefficients and obtain

$$
(-1)^{m(d-1)+(l c-1)+m_{K}\left(d_{K}-1\right)} \epsilon_{M} \sum_{\gamma \in \Sigma_{0}} \phi_{\mathrm{t}}\left(\zeta^{\gamma}\right)=(-1)^{l-1} \epsilon_{A} \sum_{\gamma \in \Sigma_{0}} \xi_{\mathrm{t}}\left(\zeta^{\gamma}\right) .
$$

Re-writing in terms of the finer symplectic invariants $\epsilon_{M}^{j}=\epsilon_{M}^{j}\left(\boldsymbol{\mu}_{E}\right)$ etc., this equation becomes

$$
(-1)^{m(d-1)+(l c-1)+m_{K}\left(d_{K}-1\right)} \epsilon_{M}^{0} \sum_{\gamma \in \Sigma_{0}} \epsilon_{M}^{1} \phi_{\mathrm{t}}\left(\zeta^{\gamma}\right)=(-1)^{l-1} \epsilon_{A}^{0} \sum_{\gamma \in \Sigma_{0}} \epsilon_{A}^{1} \xi_{\mathrm{t}}\left(\zeta^{\gamma}\right) .
$$

We can vary the character $\xi_{\mathrm{t}}$ over all the tamely ramified characters of $E^{\times}$, subject to the pair $\left(E / E_{0}, \xi_{\mathrm{t}}\right)$ being admissible ( $c f .4 .3$ Remark 2). There will always exist a tamely ramified admissible pair $\left(E / E_{0}, \phi_{\mathrm{t}}\right)$ for which this last relation holds, with $\zeta$ ranging over all $\Sigma$-regular elements of $\boldsymbol{\mu}_{K}$. We deduce from 2.3 Corollary of [14] that:

Lemma. The characters $\epsilon_{M}^{1} \phi_{\mathrm{t}}, \epsilon_{A}^{1} \xi_{\mathrm{t}}$ of $\mathbb{k}_{E}^{\times}$lie in the same $\Sigma_{0}$-orbit, and

$$
(-1)^{m(d-1)+(l c-1)+m_{K}\left(d_{K}-1\right)} \epsilon_{M}^{0}=(-1)^{l-1} \epsilon_{A}^{0} .
$$

The formula (6.7.3) can be re-written as

$$
\epsilon_{M}^{0}\left(\boldsymbol{\mu}_{E}\right) \epsilon_{A}^{0}\left(\boldsymbol{\mu}_{E}\right)=(-1)^{m(d-1)+m_{K}\left(d_{K}-1\right)+l(c-1)} .
$$

We have

$$
m_{K} d_{K}=n /[K: F], \quad l c=n /\left[E_{0}: F\right],
$$

while (as in 2.1)

$$
d_{K}=d /(d,[K: F]), \quad c=d /\left(d,\left[E_{0}: F\right]\right) .
$$


6.8. Adjusting our choices within Galois orbits, we can assume that the character $\phi$ satisfies

$$
\phi\left|U_{E}=\epsilon_{M}^{1} \epsilon_{A}^{1} \xi\right| U_{E} .
$$

Let $\left(E^{\prime} / F, \xi^{\prime}\right)$ be an admissible pair attached to $\pi$. The $F$-isomorphism class of $(E / F, \phi)$ is uniquely determined by $\tau$ [11] 2.3, and hence also by $\pi$. In other words, $\left(E^{\prime} / F, \xi^{\prime}\right)$ must give rise to the same pair $(E / F, \phi)$ as $(E / F, \xi)$. Thus $E^{\prime}$ is $F$-isomorphic to $E$, and we may as well take $E=E^{\prime}$. The relation (6.8.1) implies that the 1-pairs $\left(E / F, \xi \mid U_{E}^{1}\right),\left(E / F, \phi \mid U_{E}^{1}\right)$ and $\left(E / F, \xi^{\prime} \mid U_{E}^{1}\right)$ are all $F$ isomorphic. We may assume that they are the same. It is the restriction $\xi \mid U_{E}^{1}$ which determines the symplectic sign character $\epsilon_{A}^{1}$, so (6.8.1) now implies that the pair $\left(E / F, \xi^{\prime} \mid U_{E}\right)$ is $F$-isomorphic to $\left(E / F, \xi \mid U_{E}\right)$ in the obvious sense. Again, we may assume they are the same. It follows that $\xi^{\prime}=\chi \xi$, for some unramified character of $E^{\times}$. This implies $\tau={ }_{F} \Pi_{\chi \phi}={ }_{F} \Pi_{\phi}$, whence $(E / F, \chi \phi)$ is isomorphic to $(E / F, \phi)$. The admissibility of $(E / F, \phi)$ implies $\chi=1$, and we have proved part (1) of the Parametrization Theorem.

We prove part (2) of the Parametrization Theorem. We have bijections

$$
\begin{aligned}
P_{n}(F) & \longrightarrow \mathcal{A}_{n}^{\mathrm{et}}(F), & \mathcal{A}_{n}^{\mathrm{et}}(F) & \longrightarrow \mathcal{A}_{m}^{\mathrm{et}}(D), \\
(E / F, \xi) & \longmapsto{ }_{F} \Pi_{\xi}, & \pi & \longmapsto j \pi,
\end{aligned}
$$

given by 2.3 Theorem of [11] and Corollary 2 of 2.8 respectively. We also have an injective map $\mathcal{A}_{m}^{\text {et }}(D) \rightarrow P_{n}(F)$ given by mapping $\pi \in \mathcal{A}_{m}^{\text {et }}(D)$ to its attached admissible pair. Composing, we get a map

$$
P_{n}(F) \longrightarrow \mathcal{A}_{n}^{\mathrm{et}}(F) \longrightarrow \mathcal{A}_{m}^{\mathrm{et}}(D) \longrightarrow P_{n}(F)
$$

which preserves restrictions to 1 -units and to $F^{\times}$. It is injective, by part (1) of the Parametrization Theorem. There are only finitely many admissible pairs $(E / F, \xi)$ with specified values for $\xi\left|F^{\times}, \xi\right| U_{E}^{1}$. Consequently the map is bijective, and the result follows.

The preceding calculations also yield the First Comparison Theorem, although we have so far excluded representations of level zero. There, the result follows from 4.2 Proposition since, in this case, the symplectic sign characters are trivial. Comparing central characters, we see that the character $\nu$ is trivial as well. 
6.9. We comment further on the formula (6.7.4) and the character $\epsilon_{M}^{1} \epsilon_{A}^{1}$ of $\mathbb{k}_{E}^{\times}$. It is shown in [14] 8.4 that the constant $\epsilon_{M}^{0}\left(\boldsymbol{\mu}_{E}\right)$ and the character $\epsilon_{M}^{1}\left(\boldsymbol{\mu}_{E}\right)$ determine each other. The same applies in general.

Theorem. The following conditions are equivalent:

(1) $p \neq 2$ and $\epsilon_{A}^{0}\left(\boldsymbol{\mu}_{E}\right)=-1$;

(2) the character $\epsilon_{A}^{1}\left(\boldsymbol{\mu}_{E}\right)$ is non-trivial.

If $f(E \mid F)$ is odd, then $\epsilon_{A}^{0}\left(\boldsymbol{\mu}_{E}\right)=+1$ and $\epsilon_{A}^{1}\left(\boldsymbol{\mu}_{E}\right)$ is trivial.

Proof. The case $p=2$ is uninteresting, since $\mathbb{k}_{E}^{\times}$has no character of order 2 . We henceforward exclude it.

We write $\ell=\mathbb{k}_{E}$ and $\Omega=\operatorname{Gal}\left(\ell / \mathbb{k}_{F}\right)$. The space $\mathfrak{V}=\mathfrak{V}(\beta, \mathfrak{A})$ in our standard notation is a $\ell$-vector space on which the group $\ell^{\times}$acts via the natural conjugation action of $\boldsymbol{\mu}_{E}$. It is therefore a direct sum of spaces of the form $\Lambda_{\omega}, \omega \in \Omega$, where $\Lambda_{\omega}$ is a one-dimensional $\ell$-vector space on which $\ell^{\times}$acts by

$$
x: \lambda \longmapsto x^{\omega} x^{-1} \lambda, \quad x \in \ell^{\times}, \lambda \in \Lambda_{\omega} .
$$

Let us write

$$
\mathfrak{V}=\bigoplus_{\omega \in \Omega} \Lambda_{\omega}^{v(\omega)}=\bigoplus_{\omega \in \Omega} \mathfrak{V}_{\omega}
$$

for various integers $v(\omega) \geqslant 0$. Since $\mathfrak{V}$ is symplectic, we have $v\left(\omega^{-1}\right)=v(\omega)$ and $v(1) \equiv 0(\bmod 2)$.

The component $\mathfrak{V}_{1}$ is hyperbolic and $\ell^{\times}$acts trivially. Therefore $t_{\ell^{\times}}^{0}\left(\mathfrak{V}_{1}\right)=+1$ and the character $t_{\ell^{\times}}^{1}\left(\mathfrak{V}_{1}\right)$ is trivial. If $\omega^{2} \neq 1$, the hyperbolic space $\mathfrak{V}_{\omega} \oplus \mathfrak{V}_{\omega^{-1}}$ has trivial $t$-invariants [14] 7.3. If $\Omega$ has odd order, there is nothing more to be said. We therefore assume the contrary.

If $\omega^{2}=1 \neq \omega$, the space $\Lambda_{\omega}$ is anisotropic symplectic and the same result gives

$$
t_{\ell^{\times}}^{0}\left(\Lambda_{\omega}\right)=-1, \quad t_{\ell^{\times}}^{1}\left(\Lambda_{\omega}\right) \neq 1 .
$$

Therefore

$$
\epsilon_{A}^{0}=t_{\ell^{\times}}^{0}(\mathfrak{V})=(-1)^{v\left(\omega_{2}\right)}, \quad \epsilon_{A}^{1}=t_{\ell^{\times}}^{1}(\mathfrak{V})=\psi_{2}^{v\left(\omega_{2}\right)},
$$

where $\omega_{2}$ is the element of $\Omega$ of order 2 and $\psi_{2}$ is the character of $\ell^{\times}$of order 2 .

All assertions have now been proved.

The theorem applies equally with $M$ in place of $A$. Therefore: 
Corollary. The character $\epsilon_{A}^{1}\left(\boldsymbol{\mu}_{E}\right) \epsilon_{M}^{1}\left(\boldsymbol{\mu}_{E}\right)$ of $\boldsymbol{\mu}_{E}$ is non-trivial if and only if $p \neq 2$ and $\epsilon_{A}^{0}\left(\boldsymbol{\mu}_{E}\right) \epsilon_{M}^{0}\left(\boldsymbol{\mu}_{E}\right)=-1$.

We recall that the value of $\epsilon_{A}^{0}\left(\boldsymbol{\mu}_{E}\right) \epsilon_{M}^{0}\left(\boldsymbol{\mu}_{E}\right)$ is given explicitly by the relations (6.7.4-6).

We recall also that the invariants $\epsilon_{M}^{j}\left(\boldsymbol{\mu}_{E}\right)$ are calculated, in terms of the admissible pair $(E / F, \xi)$, in the Ramification Theorem of [14] 8.3. These calculations and the corollary together yield explicit formulæ for $\epsilon_{A}^{j}\left(\boldsymbol{\mu}_{E}\right)$.

\section{Completion of the comparison}

We continue with the admissible pair $(E / F, \xi)$ and the central simple $F$ algebra $A \cong \mathrm{M}_{m}(D)$, where $D$ is of dimension $d^{2}$ and $[E: F]=n=m d$. We complete our determination of the tamely ramified character $\nu={ }_{D} \nu_{\xi}$ encountered in the Comparison Theorem of 6.1. To do this, we have to calculate its value at one prime element $\varpi$ of $E$. We observe that there is nothing to do in the case where $E / F$ is unramified: if $\varpi_{F}$ is a prime element of $F$, then $\nu\left(\varpi_{F}\right)=1$ because the Jacquet-Langlands correspondence preserves central characters.

7.1. We continue with the notation of $\S 6$. In particular, we use the symplectic $\Psi_{E / F}$-modules $\mathfrak{V}=\mathfrak{V}(\beta, \mathfrak{A})$ and $\mathfrak{V}_{M}=\mathfrak{V}(\delta, \mathfrak{M})$ of 6.7 . As before, $E_{0} / F$ is the minimal sub-extension of $E / F$ such that $\xi \mid U_{E}^{1}$ factors through $\mathrm{N}_{E / E_{0}}$. We prove:

Second Comparison Theorem. Let $\varpi$ be any prime element of $E_{0}$ such that $\varpi^{r} \in F$, for some integer $r$ not divisible by $p$. Let $L=F[\varpi]$, let $A_{L}$ denote the $A$-centralizer of $L$, and write $A_{L} \cong \mathrm{M}_{m_{L}}\left(D_{L}\right)$, for a central L-division algebra $D_{L}$ of dimension $d_{L}^{2}$.

The character $\nu={ }_{D} \nu_{\xi}$ of $E^{\times}$then satisfies

$$
(-1)^{m(d-1)} \nu(\varpi)=(-1)^{m_{L}\left(d_{L}-1\right)} \epsilon_{M}(\varpi) \epsilon_{A}(\varpi),
$$

where

$$
\epsilon_{M}(\varpi)=t_{\langle\varpi\rangle}\left(\mathfrak{V}_{M}\right), \quad \epsilon_{A}(\varpi)=t_{\langle\varpi\rangle}(\mathfrak{V}) .
$$

The proof will occupy the remainder of the section.

Observe that the condition on $\varpi$ can be rephrased as follows: the group $\left\langle\varpi, \boldsymbol{\mu}_{E}\right\rangle$, generated by $\varpi$ and $\boldsymbol{\mu}_{E}$, contains a prime element $\varpi_{F}$ of $F$. 
7.2. Before proceeding to the proof, we point out a couple of consequences. By definition, the $\epsilon$-invariants appearing in our formulæ for ${ }_{D} \nu_{\xi}$ depend only on the simple stratum $[\mathfrak{A}, \ell, 0, \beta]$ determined by the admissible pair $(E / F, \xi)$. This, in turn, is constructed from the restriction $\xi \mid U_{E}^{1}$. Consequently:

Corollary 1. For $i=1,2$, let $\left(E / F, \xi_{i}\right)$ be an admissible pair with $[E: F]=n$. If $\xi_{1}^{-1} \xi_{2}$ is tamely ramified, then ${ }_{D} \nu_{\xi_{1}}={ }_{D} \nu_{\xi_{2}}$.

We remark that the quantities $\epsilon_{A}(\varpi), \epsilon_{M}(\varpi)$ are amenable to calculation, in terms of the structure of the admissible pair $(E / F, \xi)$, using the methods of [14] $\S 7, \S 8$. We shall not pursue the matter here beyond pointing out that, using a more elaborate version of the argument of 5.6 along with the analysis in [14] $\S 7$ and 8.5 , one obtains:

Corollary 2. Let $m \geqslant 1$ be an integer. Let $D, D^{\prime}$ be central $F$-division algebras such that $\operatorname{dim}_{F} D=\operatorname{dim}_{F} D^{\prime}=d^{2}, d \geqslant 1$. If $(E / F, \xi)$ is an admissible pair in which $[E: F]=m d$, then

(1) ${ }_{D} \nu_{\xi}={ }^{\prime} \nu_{\xi}$, and

(2) if $\boldsymbol{j}: \mathcal{A}_{m}^{\mathrm{et}}(D) \rightarrow \mathcal{A}_{m}^{\mathrm{et}}\left(D^{\prime}\right)$ is the Jacquet-Langlands correspondence, then

$$
\boldsymbol{j}\left({ }_{D} \Pi_{\xi}\right)={ }_{D^{\prime}} \Pi_{\xi}
$$

7.3. We start the proof of the Second Comparison Theorem of 7.1.

We take $\pi={ }_{D} \Pi_{\xi}$ and $\tau={ }_{F} \Pi_{\nu \xi}$ as before, so that $\pi=j_{A}(\tau)$. In particular, $\pi$ contains the extended maximal simple type $(\boldsymbol{J}, \Lambda)$, giving rise to the admissible pair $(E / F, \xi)$. We compare the character values $\operatorname{tr} \pi(\varpi u), \operatorname{tr} \tau(\varpi u)$, for an element $u$ of ${ }_{\mathrm{u}}\left(G_{L}\right)_{\mathrm{reg}}^{\mathrm{ell}}$.

Proposition. Let $x \in G$ and suppose that $\operatorname{tr} \Lambda\left(x^{-1} \varpi u x\right) \neq 0$. There then exists $y \in x \boldsymbol{J}$ such that $y^{-1} \varpi y \in E^{\times}$. For any such $y$, we have $y^{-1} u y \in J^{0}$.

Proof. The proof is exactly parallel to that of 6.1 Proposition of [14], so we omit the details.

Let $L=F[\varpi]$, let $\Sigma_{L}=\operatorname{Gal}(E / L)$ and let $G_{L}$ be the $G$-centralizer of $L$. The extension $E / L$ is unramified, so any $F$-embedding $L \rightarrow E$ extends to 
an $F$-automorphism of $E$. The element $y$ of the proposition therefore lies in $G_{L} N_{G}\left(E^{\times}\right)$and we have

$$
\operatorname{tr} \pi(\varpi u)=\sum_{x \in G_{L}} \sum_{G\left(E^{\times}\right) \boldsymbol{J} / \boldsymbol{J}} \operatorname{tr} \Lambda\left(x^{-1} \varpi u x\right) .
$$

We need to clarify the nature of the coset space $G_{L} N_{G}\left(E^{\times}\right) \boldsymbol{J} / \boldsymbol{J}$. We set $\Upsilon=$ $N_{G}\left(E^{\times}\right) / E^{\times}=\operatorname{Aut}(E \mid F)$. Take the subgroup $\Sigma_{\mathrm{t}}$ of $\Sigma_{0}$ as in 6.3. We choose representatives $t_{\alpha} \in N_{G}\left(E^{\times}\right), \alpha \in \Upsilon$, as in 6.3.

Lemma. There is a canonical bijection

$$
\begin{aligned}
\Sigma_{L} \backslash \Upsilon / \Sigma_{\mathrm{t}} & \longrightarrow G_{L} \backslash G_{L} N_{G}\left(E^{\times}\right) \boldsymbol{J} / \boldsymbol{J}, \\
\Sigma_{L} \alpha \Sigma_{\mathrm{t}} & \longmapsto G_{L} t_{\alpha} \boldsymbol{J} .
\end{aligned}
$$

Proof. We remarked in the proof of 6.3 Lemma that the image of $\boldsymbol{J} \cap N_{G}\left(K^{\times}\right)$ in $\Sigma=\operatorname{Gal}(K / F)$ is the subgroup $\Sigma_{\mathrm{t}}$. It follows that the image of $\boldsymbol{J} \cap N_{G}\left(E^{\times}\right)$ in $\Upsilon$ is the subgroup $\Sigma_{\mathrm{t}}$ again, viewed as a subgroup of $\Sigma_{0}=\operatorname{Gal}\left(E / E_{0}\right)$. Thus we have a bijection $\Upsilon / \Sigma_{\mathrm{t}} \rightarrow N_{G}\left(E^{\times}\right) \boldsymbol{J} / \boldsymbol{J}$ given by $\alpha \Sigma_{\mathrm{t}} \mapsto t_{\alpha} \boldsymbol{J}, \alpha \in \Upsilon$.

For $\alpha \in \Upsilon$, the double coset $\Sigma_{L} \alpha \Sigma_{\mathrm{t}}$ is the disjoint union of the cosets $\sigma \alpha \Sigma_{\mathrm{t}}$, where $\sigma$ ranges over a set of representatives for $\Sigma_{L} /\left(\alpha \Sigma_{\mathrm{t}} \alpha^{-1} \cap \Sigma_{L}\right)$. Under the map $\gamma \mapsto t_{\gamma}$, all of these cosets have image contained in $G_{L} t_{\alpha} \boldsymbol{J}$. The map (7.3.2) is therefore well-defined and surjective.

Let $\alpha, \beta \in \Upsilon$, and suppose that $t_{\alpha}=g t_{\beta} j$, with $g \in G_{L}$, and $j \in \boldsymbol{J}$. Since $\boldsymbol{J}=E^{\times} J^{0}$, we may as well assume $j \in J^{0}$. The element $j$ conjugates $\varpi^{\beta}$ to $\varpi^{\alpha}$. We write $\varpi^{\alpha}=\zeta_{\alpha} \varpi, \varpi^{\beta}=\zeta_{\beta} \varpi$, for roots of unity $\zeta_{\alpha}, \zeta_{\beta} \in \boldsymbol{\mu}_{K}$. We have $j^{-1} \varpi j \equiv \varpi\left(\bmod J^{1}\right)$, so $j^{-1} \zeta_{\alpha} j \equiv \zeta_{\beta}\left(\bmod J^{1}\right)$. Any such conjugation, realized by an element of $J^{0}$, is given by an element $\sigma$ of $\Sigma_{\mathrm{t}}$. Therefore $\alpha \sigma \beta^{-1}$ fixes $\varpi$, so $\alpha \sigma \beta^{-1} \in \Sigma_{L}$. In other words, $\alpha \in \Sigma_{L} \beta \Sigma_{\mathrm{t}}$; this implies that the map (7.3.2) is injective and completes the proof.

We therefore decompose the character formula (7.3.1) as

$$
\operatorname{tr} \pi(\varpi u)=\sum_{\alpha \in \Sigma_{L} \backslash \gamma / \Sigma_{\mathrm{t}}} \operatorname{tr} \pi(\varpi u ; \alpha),
$$


where

$$
\begin{aligned}
\operatorname{tr} \pi(\varpi u ; \alpha) & =\sum_{y \in G_{L} t_{\alpha} J / J} \operatorname{tr} \Lambda\left(y^{-1} \varpi u y\right) \\
& =\sum_{y \in G_{L}^{\alpha} \boldsymbol{J} / \boldsymbol{J}} \operatorname{tr} \Lambda\left(y^{-1} \varpi^{\alpha} u^{\alpha} y\right) .
\end{aligned}
$$

7.4. We examine the function $\operatorname{tr} \pi(\varpi u ; \alpha)$ of (7.3.4). Write $Z=L^{\alpha}$, so that $G_{L}^{\alpha}=G_{Z}=A_{Z}^{\times}$is the $G$-centralizer of $Z^{\times}$.

Set $\mathfrak{A}_{Z}=\mathfrak{A} \cap A_{Z}$. We recall from 3.2 Proposition that the stratum $\left[\mathfrak{A}_{Z}, \ell, 0, \beta\right]$ is simple, $H^{1}\left(\beta, \mathfrak{A}_{Z}\right)=H^{1}(\beta, \mathfrak{A}) \cap G_{Z}$ and $J^{k}\left(\beta, \mathfrak{A}_{Z}\right)=J^{k}(\beta, \mathfrak{A}) \cap G_{Z}, k=0,1$. We use the obvious abbreviations

$$
H_{Z}^{1}=H^{1}\left(\beta, \mathfrak{A}_{Z}\right), \quad J_{Z}^{k}=J^{k}\left(\beta, \mathfrak{A}_{Z}\right),
$$

for $k=0,1$.

The character $\theta_{Z}=\theta \mid H^{1}\left(\beta, \mathfrak{A}_{Z}\right)$ lies in $\mathcal{C}\left(\mathfrak{A}_{Z}, \beta, \psi_{Z}\right)$. We consider a maximal simple type $\left(J_{Z}^{0}, \lambda_{Z}\right)$ in $G_{Z}$ containing $\theta_{Z}$. We recall (2.6) that $\boldsymbol{J}\left(\lambda_{Z}\right)$ denotes the $G_{Z}$-normalizer of $\lambda_{Z}$ and that, in the same notation, the group we have been calling $\boldsymbol{J}$ is $\boldsymbol{J}(\lambda)$.

Lemma. Using the preceding notation, we have $\boldsymbol{J}\left(\lambda_{Z}\right) \supset \boldsymbol{J}(\lambda) \cap G_{Z}$. Moreover, $\boldsymbol{J}\left(\lambda_{Z}\right)=\boldsymbol{J}(\lambda) \cap G_{Z}$ if and only if $\delta_{0}\left(\lambda_{Z}\right)=[E: Z]$.

Proof. Since, by hypothesis, $\delta_{0}(\lambda)=[E: F]=n$, we have $\boldsymbol{J}(\lambda)=E^{\times} J^{0}$, giving $\boldsymbol{J}(\lambda) \cap G_{Z}=E^{\times} J_{Z}^{0}$. The lemma thus follows from (2.6.1).

7.5. Bearing 7.4 Lemma in mind, we write $\boldsymbol{J}_{Z}=\boldsymbol{J} \cap G_{Z}$. Thus $G_{Z} \boldsymbol{J} / \boldsymbol{J}=$ $G_{Z} / \boldsymbol{J}_{Z}$ and we obtain

$$
\operatorname{tr} \pi(\varpi u ; \alpha)=\sum_{y \in G_{Z} / J_{Z}} \operatorname{tr} \Lambda\left(\varpi^{\alpha} y^{-1} u^{\alpha} y\right)
$$

The element $y^{-1} u^{\alpha} y$ lies in $J^{0}$, it commutes with $\varpi^{\alpha}$, and it is pro-unipotent (7.3 Proposition).

We factorize $\Lambda$ in the form $\Lambda=\Lambda_{\mathrm{t}} \otimes \Lambda_{\mathrm{w}}$, relative to a prime element $\varpi_{F}$ of $F$ lying in $\left\langle\boldsymbol{\mu}_{E}, \varpi\right\rangle$ (cf. 7.1). We so obtain

$$
\operatorname{tr} \Lambda\left(\varpi^{\alpha} y^{-1} u^{\alpha} y\right)=\operatorname{tr} \Lambda_{\mathrm{t}}\left(\varpi^{\alpha} y^{-1} u^{\alpha} y\right) \cdot \operatorname{tr} \Lambda_{\mathrm{w}}\left(\varpi^{\alpha} y^{-1} u^{\alpha} y\right) .
$$


We first control the contribution from the "wild" factor $\operatorname{tr} \Lambda_{\mathrm{w}}$. The element $y^{-1} u^{\alpha} y$ lies in a subgroup $\mathcal{J}$ of $J^{0}$, containing $J^{1}$ and normalized by $\varpi^{\alpha}$, such that $\mathcal{J} / J^{1}$ is unipotent. In particular, $\mathcal{J}$ is a pro- $p$ group. Using the Glauberman correspondence directly, we conclude that there is a unique irreducible representation $\vartheta$ of $\mathcal{J}_{Z}=\mathcal{J} \cap J_{Z}^{0}$ and a sign $\epsilon$ such that

$$
\operatorname{tr} \Lambda_{\mathrm{w}}\left(\varpi^{\alpha} t\right)=\epsilon \operatorname{tr} \vartheta(t),
$$

for all $t \in \mathcal{J}_{Z}$. The relation (7.5.1) holds, in particular, for $t \in J_{Z}^{1}$. Therefore $\vartheta \mid J_{Z}^{1} \cong{ }_{Z} \eta_{\xi}$, and so

$$
\epsilon=\epsilon_{A}\left(\varpi^{\alpha}\right)=t_{\left\langle\varpi^{\alpha}\right\rangle}(\mathfrak{V}), \quad \mathfrak{V}=\mathfrak{V}(\beta, \mathfrak{A}) .
$$

Wide Extension Lemma. There is a wide extension $\kappa_{Z}$ of ${ }_{Z} \eta_{\xi}$ such that $\vartheta \cong \kappa_{Z} \mid \mathcal{J}_{Z}$

The proof is given in Appendix 2.

7.6. We first apply the Wide Extension Lemma of 7.5 to the case $\alpha=1$. We obtain

$$
\operatorname{tr} \Lambda\left(\varpi y^{-1} u y\right)=\epsilon_{A}(\varpi) \xi(\varpi) \operatorname{tr} \Lambda_{\mathrm{t}}\left(y^{-1} u y\right) \cdot \operatorname{tr} \kappa_{L}\left(y^{-1} u y\right),
$$

where $\kappa_{L}$ is a wide extension of ${ }_{L} \eta_{\xi}$ : the choice of $\kappa_{L}$ is immaterial (2.5.5).

Proposition. The pair $(E / L, \xi)$ is admissible. Write $A_{L} \cong \mathrm{M}_{m_{L}}\left(D_{L}\right)$, for a central L-division algebra $D_{L}$, and set $\pi_{L}={ }_{D_{L}} \Pi_{\xi}$. We then have

$$
\operatorname{tr} \pi(\varpi u ; 1)=\epsilon_{A}(\varpi) \xi(\varpi) \operatorname{tr} \pi_{L}(u) .
$$

Proof. The first assertion is clear. There is an extended maximal simple type $\left(\boldsymbol{J}(L), \Lambda_{L}\right)$ in $G_{L}$, containing the simple character $\theta_{L}$ and with attached admissible pair $(E / L, \xi)$. The group $\boldsymbol{J}(L)$ is $\boldsymbol{J}_{L}=\boldsymbol{J} \cap G_{L}$ by 7.4 Lemma. The result then follows from (7.6.1).

7.7. We consider the general term

$$
\operatorname{tr} \pi(\varpi u ; \alpha)=\sum_{y \in G_{Z} / J_{Z}} \operatorname{tr} \Lambda\left(\varpi^{\alpha} y^{-1} u^{\alpha} y\right),
$$

where $\alpha \in \Upsilon$ and $Z=L^{\alpha}=F\left[\varpi^{\alpha}\right]$. We have

$$
\operatorname{tr} \Lambda\left(\left(\varpi^{\alpha} y^{-1} u^{\alpha} y\right)=\epsilon_{A}\left(\varpi^{\alpha}\right) \operatorname{tr} \kappa_{Z}\left(y^{-1} u^{\alpha} y\right) \operatorname{tr} \Lambda_{\mathrm{t}}\left(\varpi^{\alpha} y^{-1} u^{\alpha} y\right) .\right.
$$


Lemma 1. There is a collection $\Lambda_{\mathrm{t}, i}, 1 \leqslant i \leqslant r$, of extended maximal simple types of level zero in $G_{Z}$ such that

$$
\operatorname{tr} \Lambda_{\mathrm{t}}\left(\varpi^{\alpha} j\right)=(-1)^{m-m_{L}} \sum_{i=1}^{r} \operatorname{tr} \Lambda_{\mathrm{t}, i}\left(\varpi^{\alpha} j\right),
$$

for every pro-unipotent element $j$ of $J_{Z}^{0}$. Moreover, if $\lambda_{\mathrm{t}, i}=\Lambda_{\mathrm{t}, i} \mid J_{Z}^{0}$, then $\delta_{0}\left(\lambda_{\mathrm{t}, i}\right)=\left[E: E_{0} Z\right]$, for all $i$.

Proof. The result can be read off from the discussion in paragraph 6.10 of [14].

The type $\Lambda_{\mathrm{t}, i}$ is thus determined by a tamely ramified admissible pair of the form $\left(E / Z E_{0}, \psi_{i}\right)$. We set $\chi_{i}=\psi_{i} \cdot \xi_{\mathrm{w}}$; this gives a family of admissible pairs $\left(E / Z, \chi_{i}\right), 1 \leqslant i \leqslant r$. Applying Lemma 1 and 7.4 Lemma, we may argue as in 7.6 Proposition to get

$$
\operatorname{tr} \pi(\varpi u ; \alpha)=(-1)^{m-m_{L}} \epsilon_{A}\left(\varpi^{\alpha}\right) \sum_{i=1}^{r} \operatorname{tr}_{D_{Z}} \Pi_{\chi_{i}}\left(\varpi^{\alpha} u^{\alpha}\right) .
$$

It is more convenient to re-write this relation in the form

$$
\begin{aligned}
\operatorname{tr} \pi\left(\varpi u ; \alpha^{-1}\right) & =(-1)^{m-m_{L}} \epsilon_{A}\left(\varpi^{\alpha^{-1}}\right) \sum_{i=1}^{r} \operatorname{tr}_{D_{L}} \Pi_{\chi_{i}^{\alpha}}(\varpi u) \\
& =(-1)^{m-m_{L}} \epsilon_{A}\left(\varpi^{\alpha^{-1}}\right) \xi^{\alpha}(\varpi) \sum_{i=1}^{r} \operatorname{tr}_{D_{L}} \Pi_{\chi_{i}^{\alpha}}(u),
\end{aligned}
$$

for $\alpha \in \Sigma_{\mathrm{t}} \backslash \Upsilon / \Sigma_{L}$.

Consider the admissible pair $\left(E / L, \chi_{i}^{\alpha}\right)$. We recall that $\xi \mid U_{E}^{1}=\xi_{0} \circ \mathrm{N}_{E / E_{0}}$, the 1-pair $\left(E_{0} / F, \xi_{0}\right)$ being admissible.

\section{Lemma 2.}

(1) The character $\chi_{i}^{\alpha} \mid U_{E}^{1}$ factors through $\mathrm{N}_{E / L E_{0}^{\alpha}}$, and $L E_{0}^{\alpha} / L$ is the minimal sub-extension of $E / L$ with this property.

(2) The restriction $\chi_{i}^{\alpha} \mid U_{E}^{1}$ is of the form $\xi_{0}^{\alpha} \circ \mathrm{N}_{L E_{0}^{\alpha} / E_{0}^{\alpha}}$.

(3) The 1-pair $\left(L E_{0}^{\alpha} / L, \xi_{0}^{\alpha} \circ \mathrm{N}_{\left.L E_{0}^{\alpha} / E_{0}^{\alpha}\right)}\right.$ is L-isomorphic to $\left(E_{0} / L, \xi_{0}\right)$ if and only if $\alpha \in \Sigma_{L}$. 
Proof. By definition, $\chi_{i}\left|U_{E}^{1}=\xi\right| U_{E}^{1}$, so we need only treat the character $\xi$. Looking back at the proof of [11] A.1 Lemma (stated above as 3.3 Lemma), the field $E_{0}$ arises as follows. We choose a finite extension $\widetilde{E} / E$ such that $\widetilde{E} / F$ is Galois and set $\Gamma=\operatorname{Gal}(\widetilde{E} / F)$. If $\Delta$ is the subgroup of $\Gamma$ which fixes $\xi \mid U_{E}^{1}$, the field $E_{0}$ is $\widetilde{E}^{\Delta}$. The character $\xi_{0}$ is then uniquely determined by $\xi \mid U_{E}^{1}$.

Parts (1) and (2) of the lemma follow immediately. In part (3), one implication is obvious. In the opposite direction, the extension $E / L$ is unramified, so if $L E_{0}^{\alpha}$ is $L$-isomorphic to $E_{0}$ then $L E_{0}^{\alpha}=E_{0}$ and the isomorphism is realized by an $L$-automorphism of $E_{0}$, that is, by an element $\sigma$ of $\Sigma_{L}$. If the pairs are to be isomorphic, this automorphism must carry $\xi_{0}^{\alpha}$ to $\xi_{0}$. This implies that $\sigma^{-1} \alpha$ fixes $\xi_{0}$, whence $\sigma^{-1} \alpha \in \Sigma_{0}$ and $\alpha \in \Sigma_{L}$, as required.

Remark. There is the following more precise version of part (3) of the lemma. If $\alpha, \beta \in \Upsilon$, the pairs

$$
\left(L E_{0}^{\alpha} / L, \xi_{0}^{\alpha} \circ \mathrm{N}_{L E_{0}^{\alpha} / E_{0}^{\alpha}}\right), \quad\left(L E_{0}^{\beta} / L, \xi_{0}^{\beta} \circ \mathrm{N}_{L E_{0}^{\beta} / E_{0}^{\beta}}\right)
$$

are $L$-isomorphic if and only if $\Sigma_{0} \alpha \Sigma_{L}=\Sigma_{0} \beta \Sigma_{L}$. We do not need this fact, so we omit the proof.

7.8. We return, for the moment, to a general situation. If $\Phi \in \mathcal{A}_{m}^{\mathrm{et}}(D)$, we may write $\Phi={ }_{D} \Pi_{\vartheta}$, for an admissible pair $(H / F, \vartheta)$. This pair determines an admissible 1-pair $\left(H_{0} / F, \vartheta_{0}\right)$, the $F$-isomorphism class of which is determined by $\Phi$. We extend our terminology and say that $\left(H_{0} / F, \vartheta_{0}\right)$ is the 1-pair attached to $\Phi$.

Linear Independence Lemma. Let $\pi_{1}, \pi_{2}, \ldots, \pi_{r} \in \mathcal{A}_{m}^{\mathrm{et}}(D)$. Let $\left(E_{i} / F, \gamma_{i}\right)$ be an admissible 1-pair attached to $\pi_{i}, 1 \leqslant i \leqslant r$. Suppose that $\left(E_{i} / F, \gamma_{i}\right)$ is not $F$-isomorphic to $\left(E_{j} / F, \gamma_{j}\right)$ when $i \neq j$. The functions $u \mapsto \operatorname{tr} \pi_{i}(u), 1 \leqslant i \leqslant r$, on $G_{\mathrm{u}}^{\mathrm{ell}}$ then form a linearly independent set.

Proof. Let $H$ be a central $F$-division algebra of dimension $n^{2}$ and let $\boldsymbol{j}_{H}$ denote the Jacquet-Langlands correspondence $\mathcal{A}_{m}^{\text {et }}(D) \rightarrow \mathcal{A}_{1}^{\text {et }}(H)$. The admissible 1 -pair attached to $\boldsymbol{j}_{H}\left(\pi_{i}\right)$ is $\left(E_{i} / F, \gamma_{i}\right), 1 \leqslant i \leqslant r$, by the First Comparison Theorem of 6.1. We may therefore assume we are in the case $m=1$. So, until the end of the proof of the Linear Independence Lemma, $A$ is a division algebra. 
Lemma. Suppose $A$ is a division algebra, and let $\theta_{i}$ be a simple character occurring in $\pi_{i}, 1 \leqslant i \leqslant r$. If the characters $\theta_{i}, \theta_{j}$ intertwine in $G$, then $i=j$.

Proof. If $\theta_{i}$ intertwines with $\theta_{j}$, then $\theta_{i}$ is $G$-conjugate to $\theta_{j}$ [4] (11.2.9). The 1pairs $\left(E_{i} / F, \gamma_{i}\right),\left(E_{j} / F, \gamma_{j}\right)$ are then $F$-isomorphic, as follows from the Parametrization Theorem 6.1 and the construction of the attached pair.

Let $\mathcal{H}(G)$ be the space of locally constant, compactly supported functions $G \rightarrow \mathbb{C}$, viewed as convolution algebra relative to a choice of Haar measure on $G$. Let $e_{i} \in \mathcal{H}(G)$ be the idempotent corresponding to the simple character $\theta_{i}$. Thus $e_{i}$ has support contained in $U_{A}^{1}=U_{\mathfrak{o}_{A}}^{1}$ and, if $(\pi, V)$ is an admissible representation of $G$, we have

$$
\int_{G} \operatorname{tr} \pi(g) e_{i}(g) d g=\operatorname{dim} V^{\theta_{i}}
$$

where $V^{\theta_{i}}$ is the $\theta_{i}$-isotypic subspace of $V$.

Since $A$ is a division algebra, the set ${ }_{\mathrm{u}} G_{\mathrm{reg}}^{\mathrm{ell}}$ is open dense in $U_{A}^{1}$. Consequently, if we have a linear dependence relation

$$
\sum_{i=1}^{r} a_{i} \operatorname{tr} \pi_{i}(u)=0, \quad u \in{ }_{\mathrm{u}} G_{\mathrm{reg}}^{\mathrm{ell}},
$$

we may integrate it against $e_{j}$ to get

$$
\begin{aligned}
0 & =\sum_{i=1}^{r} a_{i} \int_{U_{A}^{1}} \operatorname{tr} \pi_{i}(u) e_{j}(u) d u \\
& =\sum_{i=1}^{r} a_{i} \int_{G} \operatorname{tr} \pi_{i}(g) e_{j}(g) d g=c_{j} a_{j},
\end{aligned}
$$

where $c_{j}>0$ is the dimension of the $\theta_{j}$-isotypic subspace of $\pi_{j}$. Thus $a_{j}=0$, as required.

7.9. We return to the context of 7.7 and consider the representation $\tau={ }_{F} \Pi_{\nu \xi}$. As a special case of (7.6.1), we have

$$
\operatorname{tr} \tau(\varpi u ; 1)=\epsilon_{M}(\varpi) \nu \xi(\varpi) \operatorname{tr} \tau_{L}(u),
$$

where $\tau_{L}={ }_{L} \Pi_{\nu \xi}$ and $u$ ranges over the pro-unipotent, elliptic regular elements of the $\mathrm{GL}_{n}(F)$-centralizer $\mathrm{GL}_{n}(F)_{L}$ of some copy of $L$ embedded in $\mathrm{M}_{n}(F)$. From 
7.7 Lemma, the Linear Independence Lemma of 7.8 and the defining relation $\pi=\boldsymbol{j}_{A} \tau$, we obtain

$$
\operatorname{tr} \pi(\varpi u ; 1)=(-1)^{m(d-1)} \operatorname{tr} \tau(\varpi u ; 1)
$$

and hence

$$
(-1)^{m(d-1)} \epsilon_{M}(\varpi) \nu \xi(\varpi) \operatorname{tr} \tau_{L}(u)=\epsilon_{A}(\varpi) \xi(\varpi) \operatorname{tr} \pi_{L}(u),
$$

this relation being valid for all $u \in{ }_{\mathrm{u}}\left(G_{L}\right)_{\mathrm{reg}}^{\mathrm{ell}}$. (Note that, in the left hand side, $u$ denotes an element of $\mathrm{GL}_{n}(F)_{L}$ associate to $u$.)

We recall that $A_{L} \cong \mathrm{M}_{m_{L}}\left(D_{L}\right)$, for an $L$-division algebra $D_{L}$ of dimension $d_{L}^{2}$. We let $u \rightarrow 1$ in the right hand side of (7.9.1). The associate element $u$ in the left hand side then also tends to 1 (1.6 Proposition). From 1.5 Theorem (1), we get

$$
(-1)^{m(d-1)} \epsilon_{M}(\varpi) \nu \xi(\varpi)(-1)^{m_{L} d_{L}-1} c_{\tau_{L}}=\epsilon_{A}(\varpi) \xi(\varpi)(-1)^{m_{L}-1} c_{\pi_{L}},
$$

for certain positive constants $c_{\tau_{L}}, c_{\pi_{L}}$. These cancel, since every other factor is a root of unity. Therefore

$$
(-1)^{m(d-1)} \nu(\varpi)=(-1)^{m_{L}\left(d_{L}-1\right)} \epsilon_{M}(\varpi) \epsilon_{A}(\varpi),
$$

as required to prove the theorem.

\section{APPEndix 1. \\ Parametric degree and formal degree}

Here we prove Theorem 2.8, which connects the normalized formal degree $\operatorname{deg} \pi$ and the parametric degree $\delta(\pi)$ of a representation $\pi \in \mathcal{A}_{m}^{\square}(D)$. This is an exercise, parallel to [9] and generalizing $\S 7.7$ of [17]. The novelty derives from the Hecke algebra calculations of [41], [24].

A1.1. Let $G=\mathrm{GL}_{m}(D), A=\mathrm{M}_{m}(D)$, where $D$ is a central $F$-division algebra of dimension $d^{2}, d \geqslant 1$. We set $n=m d$. We recall a well-known property of the essentially square-integrable representations of $G$ - see for instance [21] Théorème B.2.b - but we give a rather different proof. 
Proposition. Let $\pi \in \mathcal{A}_{m}^{\square}(D)$. There is then a positive divisor $r$ of $m$ and an irreducible cuspidal representation $\pi_{0}$ of $\mathrm{GL}_{m / r}(D)$ such that the cuspidal support of $\pi$ consists of unramified twists of $\pi_{0}$.

Proof. Replacing $\pi$ by $\chi \pi$, where $\chi$ is a character of $F^{\times}$, changes nothing. We may therefore assume $\pi$ to be square-integrable. It follows, from an argument identical to the proof Proposition 8.5.10 of [17], that $\pi$ cannot be of the form $\operatorname{Ind}_{P}^{G} \tau$, where $P$ is a proper parabolic subgroup of $G$ and $\tau$ is an irreducible representation of $P$ trivial on the unipotent radical of $P$. However, if the cuspidal support of $\pi$ is not of the stated form, then Theorem 3.1 of [36] asserts that $\pi$ is an irreducible, parabolically induced representation of just this kind.

A1.2. It follows from Théorème 5.23 of [41] that any $\pi \in \mathcal{A}_{m}^{\square}(D)$ contains a simple type, in the sense of [40].

In order to review the definition of a simple type, it will be simpler to introduce a new scheme of notation. Let $\pi$ be an irreducible cuspidal representation of $\mathrm{GL}_{m}(D)$. Thus $\pi$ contains a maximal simple type $(J, \lambda)$. We assume initially that this type is of positive level, and use the notational scheme of 2.6 above. Thus there is a simple stratum $[\mathfrak{A}, \ell, 0, \beta]$ in $A=\mathrm{M}_{m}(D)$ so that $J=J^{0}(\beta, \mathfrak{A})$. The hereditary order $\mathfrak{A}$ is maximal among $E_{0}$-pure hereditary orders in $A$, where $E_{0}$ denotes the field $F[\beta]$. We denote by $B$ the $A$-centralizer of $E_{0}$, so that $B \cong \mathrm{M}_{l}(C)$, where $C$ is a central $E_{0}$-division algebra of dimension $c^{2}$, and $l c=$ $n /\left[E_{0}: F\right]$. The order $\mathfrak{B}=\mathfrak{A} \cap B$ is a maximal $\mathfrak{o}_{E_{0}}$-order in $B$.

The restriction of $\lambda$ to $H^{1}(\beta, \mathfrak{A})$ is a multiple of a simple character $\theta \in$ $\mathcal{C}\left(\mathfrak{A}, \beta, \psi_{F}\right)$. Let $\eta$ be the unique irreducible representation of $J^{1}(\beta, \mathfrak{A})$ contain$\operatorname{ing} \theta$, and let $\kappa$ be some wide extension of $\eta$. The representation $\lambda$ is then of the form $\lambda=\sigma \otimes \kappa$, for an irreducible representation $\sigma$ of $J(\beta, \mathfrak{A})$ trivial on $J^{1}(\beta, \mathfrak{A})$. The restriction $\sigma_{\mathfrak{B}}$ of $\sigma$ to $U_{\mathfrak{B}}=J(\beta, \mathfrak{A}) \cap B$ is trivial on $U_{\mathfrak{B}}^{1}=J^{1}(\beta, \mathfrak{A}) \cap B$. The pair $\left(U_{\mathfrak{B}}, \sigma_{\mathfrak{B}}\right)$ is a maximal simple type of level zero in the group $B^{\times}$. The representation $\sigma_{\mathfrak{B}}$ is the inflation of an irreducible cuspidal representation $\tilde{\sigma}$ of $U_{\mathfrak{B}} / U_{\mathfrak{B}}^{1} \cong \mathrm{GL}_{l}\left(\mathbb{k}_{C}\right)$.

In this scheme, we have

$$
\delta(\pi)=\delta_{0}\left(\sigma_{\mathfrak{B}}\right)\left[E_{0}: F\right], \quad t(\pi)=\delta(\pi) / e\left(E_{0} \mid F\right) .
$$

We add a further datum to this list. We fix an unramified field extension 
$E / E_{0}$, inside $B$, so that $[E: F]=n$ and $\mathfrak{B}$ is $E$-pure. Thus $\mathfrak{A}$ is $E$-pure. These conditions determine $\mathfrak{A}$ and $\mathfrak{B}$ uniquely in terms of $E$.

The modifications required for the level zero case are both clear and straightforward.

A1.3. Continuing with the notation of $\mathrm{A} 1.2$, let $r \geqslant 1$ be an integer and let $\bar{A}=\mathrm{M}_{m r}(D), \bar{G}=\mathrm{GL}_{m r}(D)$. We embed the field $E$ in $\bar{A}$, in some way, as an $F$ subalgebra. We choose an $E$-pure hereditary $\mathfrak{o}_{F}$-order $\overline{\mathfrak{A}}$ in $\bar{A}$, which is minimal relative to this property. The $F$-period of $\overline{\mathfrak{A}}$ is thus $r$ times the $F$-period of $\mathfrak{A}$, and we get a simple stratum $[\overline{\mathfrak{A}}, r \ell, 0, \beta]$ in $\bar{A}$.

Let $\bar{B}$ denote the centralizer of $E_{0}$ in $\bar{A}$ : thus $\bar{B} \cong \mathrm{M}_{l r}(C)$. We set $\overline{\mathfrak{B}}=\overline{\mathfrak{A}} \cap \bar{B}$.

The transfer process of [38] Théorème 3.53 determines, from $\theta$ and $\beta$, a simple character $\bar{\theta} \in \mathcal{C}\left(\overline{\mathfrak{A}}, \beta, \psi_{F}\right)$. Let $\bar{\eta}$ be the unique irreducible representation of $J^{1}(\beta, \overline{\mathfrak{A}})$ which contains $\bar{\theta}$. Following the procedures of [39], starting from Théorème 2.18 , we obtain from $\kappa$ a wide extension $\bar{\kappa}$ of $\bar{\eta}$.

We have $J(\beta, \overline{\mathfrak{A}})=U_{\overline{\mathfrak{B}}} J^{1}(\beta, \overline{\mathfrak{A}})$ and $U_{\overline{\mathfrak{B}}} \cap J^{1}(\beta, \overline{\mathfrak{A}})=U_{\overline{\mathfrak{B}}}^{1}$. The group $U_{\overline{\mathfrak{B}}} / U_{\overline{\mathfrak{B}}}^{1}$ is the direct product of $r$ copies of $\mathrm{GL}_{l}\left(\mathbb{k}_{C}\right)$. We let $\bar{\sigma}_{\mathfrak{B}}$ be the inflation to $U_{\overline{\mathfrak{B}}}$ of $\tilde{\sigma} \otimes \tilde{\sigma} \otimes \cdots \otimes \tilde{\sigma}$ ( $r$ factors $)$. We extend $\bar{\sigma}_{\mathfrak{B}}$ to a representation $\bar{\sigma}$ of $J(\beta, \overline{\mathfrak{A}})$ trivial on $J^{1}(\beta, \overline{\mathfrak{A}})$. We set

$$
\bar{\lambda}=\bar{\sigma} \otimes \bar{\kappa}, \quad \bar{J}=J(\beta, \overline{\mathfrak{A}}) .
$$

The representation $\bar{\lambda}$ is irreducible. The pair $(\bar{J}, \bar{\lambda})$ is an instance of a simple type in $\bar{G}$, in the sense of [40]. The property we require is [41] Théorème 5.23:

(A1.3.1) Let $\Pi$ be an irreducible smooth representation of $\bar{G}$. The following conditions are equivalent:

(1) $\Pi$ contains the simple type $(\bar{J}, \bar{\lambda})$;

(2) the cuspidal support of $\Pi$ consists of unramified twists of $\pi$.

A1.4. We recall and extend some standard material from, for example, [17] 7.7 and [18] $\S 8$. We fix an integer $r \geqslant 1$, and consider those representations $\tau \in \mathcal{A}_{r}^{\square}(F)$ which have, as cuspidal support, unramified characters of $F^{\times}$. Among these is the Steinberg representation, which we denote $\mathrm{St}_{r}^{F}$. Indeed, any other representation of this kind is an unramified twist of $\mathrm{St}_{r}^{F}$, and has the same formal degree. A calculation originating in [3] yields: 
(A1.4.1) Let $\dot{\mu}_{r}^{F}$ denote a Haar measure on $\mathrm{GL}_{r}(F) / F^{\times}$, and let $\mathcal{I}_{r}(F)$ be an Iwahori subgroup of $\mathrm{GL}_{r}(F)$. The formal degree of the Steinberg representation $\mathrm{St}_{r}^{F}$ satisfies

$$
\dot{\mu}_{r}^{F}\left(\mathcal{I}_{r}(F) F^{\times}\right) d\left(\mathrm{St}_{r}^{F}, \dot{\mu}_{r}^{F}\right)=\frac{1}{r} \frac{(q-1)^{r}}{q^{r}-1},
$$

where $q=q_{F}=\left|\mathbb{k}_{F}\right|$.

We recall that an Iwahori subgroup of $\mathrm{GL}_{r}(F)$ is the same as the unit group of a minimal hereditary $\mathfrak{o}_{F}$-order in $\mathrm{M}_{r}(F)$.

We extend this notation by letting $\mathcal{I}_{r}(D)$ denote the unit group of some minimal hereditary order in $\mathrm{M}_{r}(D)$, when $D$ is a central $F$-division algebra of dimension $d^{2}$. Again we consider the elements of $\mathcal{A}_{r}^{\square}(D)$ having unramified characters of $D^{\times}=\mathrm{GL}_{1}(D)$ as cuspidal support. Among these is the Steinberg representation $\mathrm{St}_{r}^{D}$.

Proposition. Let $\dot{\mu}_{r}^{D}$ be a Haar measure on $\mathrm{GL}_{r}(D) / F^{\times}$. The formal degree of the Steinberg representation $\mathrm{St}_{r}^{D}$ satisfies

$$
\dot{\mu}_{r}^{D}\left(\mathcal{I}_{r}(D) F^{\times}\right) d\left(\operatorname{St}_{r}^{D}, \dot{\mu}_{r}^{D}\right)=\frac{1}{d r} \frac{\left(q^{d}-1\right)^{r}}{q^{d r}-1} .
$$

Proof. Set $G=\mathrm{GL}_{r}(D)$ and let $\mathcal{H}\left(G, \mathcal{I}_{r}(D)\right)$ be the convolution algebra of compactly supported functions $G \rightarrow \mathbb{C}$ which are left- and right-invariant under translation by $\mathcal{I}_{r}(D)$. As a normalized Hilbert algebra (in the sense of [15] $\S 3$ or [22]), this is isomorphic to the affine Hecke algebra $\mathcal{H}\left(r, q_{D}\right)$ (in the notation of [17] (5.4.6)). This, in turn, is the same as $\mathcal{H}\left(\mathrm{GL}_{r}(K), \mathcal{I}_{r}(K)\right)$, where $K / F$ is an unramified field extension of degree $d$. Under the composite isomorphism $\mathcal{H}\left(G, \mathcal{I}_{r}(D)\right) \cong \mathcal{H}\left(\mathrm{GL}_{r}(K), \mathcal{I}_{r}(K)\right)$, functions with support contained in $F^{\times} \mathcal{I}_{r}(D)$ are mapped to the functions with support contained in $\left(K^{\times}\right)^{d} \mathcal{I}_{r}(K)$, where $\left(K^{\times}\right)^{d}$ denotes the group of $d$-th powers in $K^{\times}$. As in [17] 7.7 (or following the more general analysis in [15]), we get the relation

$$
\begin{aligned}
\dot{\mu}_{r}^{D}\left(\mathcal{I}_{r}(D) F^{\times}\right) d\left(\mathrm{St}_{r}^{D}, \dot{\mu}_{r}^{D}\right) & =d^{-1} \dot{\mu}_{r}^{K}\left(\mathcal{I}_{r}(K) K^{\times}\right) d\left(\mathrm{St}_{r}^{K}, \dot{\mu}_{r}^{K}\right) \\
& =\frac{1}{d r} \frac{\left(q^{d}-1\right)^{r}}{q^{d r}-1},
\end{aligned}
$$

as required. 
A1.5. We return to the situation of $\mathrm{A} 1.2-3$. Let $\Pi \in \mathcal{A}_{m r}^{\square}(D)$ have cuspidal support consisting of unramified twists of $\pi \in \mathcal{A}_{m}^{\square}(D)$. By (A1.3.1), the representation $\Pi$ contains the simple type $(\bar{J}, \bar{\lambda})$.

We consider the Hecke algebra $\mathcal{H}(\bar{G}, \bar{\lambda})$. Appealing to [40] Théorème 4.6, $\mathcal{H}(\bar{G}, \bar{\lambda})$ is isomorphic, as Hilbert algebra, to the affine Hecke algebra

$$
\mathcal{H}\left(r, q_{E}\right)=\mathcal{H}\left(\mathrm{GL}_{r}(E), \mathcal{I}_{r}(E)\right) .
$$

However, under the isomorphism $\mathcal{H}(\bar{G}, \bar{\lambda}) \rightarrow \mathcal{H}\left(\mathrm{GL}_{r}(E), \mathcal{I}_{r}(E)\right)$, functions supported in $F^{\times} \bar{J}$ become functions supported in $\left(E^{\times}\right)^{k} \mathcal{I}_{r}(E)$, where $k$ is the integer $e\left(E_{0} \mid F\right) l c / \delta_{0}\left(\sigma_{\mathfrak{B}}\right)$. This gives us the relation

$$
\dot{\mu}_{m r}^{D}\left(\bar{J} F^{\times}\right) d\left(\Pi, \dot{\mu}_{m r}^{D}\right)=\frac{\delta_{0}\left(\sigma_{\mathfrak{B}}\right)}{e\left(E_{0} \mid F\right) l c} \dot{\mu}_{r}^{E}\left(\mathcal{I}_{r}(E) E^{\times}\right) d\left(\mathrm{St}_{r}^{E}, \dot{\mu}_{r}^{E}\right) \operatorname{dim} \bar{\lambda} .
$$

From the definitions, $\delta(\pi)=\delta(\Pi)=\left[E_{0}: F\right] \delta_{0}\left(\sigma_{\mathfrak{B}}\right)$, so $(2.1 .1)$

$$
\frac{\delta_{0}\left(\sigma_{\mathfrak{B}}\right)}{e\left(E_{0} \mid F\right) l c}=t(\Pi) / n
$$

Since (A1.4.1)

$$
\dot{\mu}_{r}^{E}\left(\mathcal{I}_{r}(E) E^{\times}\right) d\left(\mathrm{St}_{r}^{E}, \dot{\mu}_{r}^{E}\right)=r^{-1} \frac{\left(q_{E}-1\right)^{r}}{q_{E}^{r}-1}
$$

we have

$$
\dot{\mu}_{m r}^{D}\left(\bar{J} F^{\times}\right) d\left(\Pi, \dot{\mu}_{m r}^{D}\right)=\frac{t(\Pi)}{n r} \frac{\left(q_{E}-1\right)^{r}}{q_{E}^{r}-1} \operatorname{dim} \bar{\lambda} .
$$

We now choose $\dot{\mu}_{m r}^{D}$ so that $d\left(\mathrm{St}_{m r}^{D}, \dot{\mu}_{m r}^{D}\right)=1$. That is,

$$
\dot{\mu}_{m r}^{D}\left(\mathcal{I}_{m r}(D) F^{\times}\right)=\frac{1}{d m r} \frac{\left(q^{d}-1\right)^{m r}}{q^{d m r}-1} .
$$

This yields

$$
\operatorname{deg} \Pi=\left(\mathcal{I}_{m r}(D) F^{\times}: \bar{J} F^{\times}\right) t(\Pi) \frac{q^{d m r}-1}{\left(q^{d}-1\right)^{m r}} \frac{\left(q_{E}-1\right)^{r}}{q_{E}^{r}-1} \operatorname{dim} \bar{\lambda} .
$$

A1.6. We now prove 2.8 Theorem. In our present scheme of notation A1.2, A1.3, it reads: 
Theorem. Let $\Pi \in \mathcal{A}_{m r}^{\square}(D)$ have cuspidal support consisting of unramified twists of a cuspidal representation $\pi$ of $\mathrm{GL}_{m}(D)$. We then have

$$
\operatorname{deg} \Pi \equiv t(\Pi) \frac{\left(q^{n r}-1\right)}{\left(q^{t(\Pi) n r / \delta(\Pi)}-1\right)} \quad\left(\bmod p^{\mathbb{Z}}\right) .
$$

Proof. We recall that $t(\Pi)=t(\pi)$ and $\delta(\Pi)=\delta(\pi)$ in our present scheme.

We proceed by simplifying the expression (A1.5.1). The generalized group index term there contributes

$$
\left(\mathcal{I}_{m r}(D) F^{\times}: \bar{J} F^{\times}\right) \equiv\left(q^{d}-1\right)^{m r}\left|\mathrm{GL}_{l}\left(\mathbb{k}_{C}\right)\right|^{-r}\left(\bmod p^{\mathbb{Z}}\right) .
$$

On the other hand,

$$
\operatorname{dim} \bar{\lambda} \equiv\left|\mathrm{GL}_{l}\left(\mathbb{k}_{C}\right)\right|^{r}\left(q_{C}^{l}-1\right)^{-r} \quad\left(\bmod p^{\mathbb{Z}}\right),
$$

so

$$
\operatorname{deg} \Pi \equiv t(\Pi)\left(q^{n r}-1\right)\left(q_{E}-1\right)^{r}\left(q_{E}^{r}-1\right)^{-1}\left(q_{C}^{l}-1\right)^{-r} \quad\left(\bmod p^{\mathbb{Z}}\right) .
$$

Now we recall that $q_{E}=q_{C}^{l}=q_{E_{0}}^{l c}=q^{n / e\left(E_{0} \mid F\right)}$, resulting in

$$
\operatorname{deg} \Pi \equiv t(\Pi)\left(q^{n r}-1\right)\left(q_{E}^{r}-1\right)^{-1} \quad\left(\bmod p^{\mathbb{Z}}\right) .
$$

In the final term $\left(q_{E}^{r}-1\right)^{-1}$, we have $q_{E}^{r}=q^{n r / e\left(E_{0} \mid F\right)}$. However,

$$
t(\Pi)=t(\pi)=\delta(\pi) / e\left(E_{0} \mid F\right)=\delta(\Pi) / e\left(E_{0} \mid F\right),
$$

yielding $n r / e\left(E_{0} \mid F\right)=n r t(\Pi) / \delta(\Pi)$. The relation (A1.6.1) has been proved.

Remark. Using (A1.3.1) and the Hecke algebra isomorphisms cited in the preceding proof, it is a straightforward matter to refine A1.1 Proposition to give a complete description of the non-cuspidal elements of $\mathcal{A}_{m}^{\square}(D)$ : the argument is identical to that of Theorem 8.3 of [18].

\section{APPENDIX 2.}

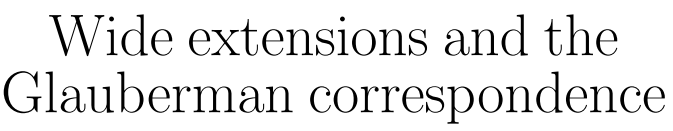

We prove the Wide Extension Lemma of 7.5. 
A2.1. We start in an abstract setting, recalling a fundamental result from [23].

We are given a finite group $T$ and a cyclic group of automorphisms $Z$ of $T$, such that $|Z|$ is relatively prime to $|T|$. We think of $Z$ as acting on $T$ by conjugation, so that we may form the semi-direct product group $Z \ltimes T=Z T$. The group $Z$ acts on the set $\operatorname{Irr} T$ of equivalence classes of irreducible representations of $T$ by $\zeta: \rho \mapsto \rho^{\zeta}, \zeta \in Z, \rho \in \operatorname{Irr} T$, where $\rho^{\zeta}$ denotes the representation $t \mapsto \rho\left(\zeta t \zeta^{-1}\right)$, $t \in T$

We denote by $T^{Z}$ the group of $Z$-fixed points in $T$, and by $\operatorname{Irr}\left(T^{Z}\right)$ the set of equivalence classes of irreducible representations of $T^{Z}$. We summarize the main points needed from [23].

(A2.1.1) Let $\zeta$ generate the group $Z$.

(1) If $\tau$ is an irreducible representation of $Z T$ such that $\tau \mid T$ is not irreducible, then

$$
\operatorname{tr} \tau(\zeta t)=0, \quad t \in T
$$

(2) Let $\rho \in(\operatorname{Irr} T)^{Z}$.

(a) There exists a unique irreducible representation $\tilde{\rho}$ of $Z T$ such that $\tilde{\rho} \mid T \cong \rho$ and $\operatorname{det} \tilde{\rho} \mid Z=1$.

(b) There is a unique irreducible representation $\rho_{Z}$ of $T^{Z}$, and a constant $\epsilon=\epsilon_{\zeta}(\rho)= \pm 1$ such that

$$
\operatorname{tr} \tilde{\rho}(\zeta t)=\epsilon \operatorname{tr} \rho_{Z}(t), \quad t \in T^{Z} .
$$

(c) The map $\rho \mapsto \rho_{Z}$ is a bijection $(\operatorname{Irr} T)^{Z} \rightarrow \operatorname{Irr}\left(T^{Z}\right)$, independent of the choice of $\zeta$.

The sign $\epsilon_{\zeta}(\rho)$ may indeed vary with both $\zeta$ and $\rho$. The bijection $(\operatorname{Irr} T)^{Z} \rightarrow$ $\operatorname{Irr}\left(T^{Z}\right)$ is what we call the Glauberman correspondence.

There is a more general version of (b).

(A2.1.2) Let $\rho \in(\operatorname{Irr} T)^{Z}$, and let $\boldsymbol{\rho}$ be a representation of $Z T$ such that $\boldsymbol{\rho} \mid T \cong$ $\rho$. There is a constant root of unity $\alpha$, of order dividing $2|Z|$, such that

$$
\operatorname{tr} \boldsymbol{\rho}(\zeta t)=\alpha \operatorname{tr} \rho_{Z}(t), \quad t \in T^{Z} .
$$

We denote by $\langle$,$\rangle the standard inner product of class functions on a finite$ group. We call the elements of $\operatorname{Irr} T \backslash(\operatorname{Irr} T)^{Z} Z$-irrelevant. 
Proposition. Let $\theta$ be a finite-dimensional representation of $T$ such that $\theta^{\zeta} \cong$ $\theta$. Let $\boldsymbol{\theta}$ be a representation of $Z T$ such that $\boldsymbol{\theta} \mid T \cong \theta$, let $\operatorname{tr}_{\zeta}(\boldsymbol{\theta})$ denote the class function $j \mapsto \operatorname{tr} \boldsymbol{\theta}(\zeta j)$ on $T^{Z}$, and let $\rho \in(\operatorname{Irr} T)^{Z}$.

(1) If $\left\langle\operatorname{tr}\left(\rho_{Z}\right), \operatorname{tr}_{\zeta}(\boldsymbol{\theta})\right\rangle=a \in \mathbb{C}$, then $\rho$ occurs in $\theta$ with multiplicity $\geqslant|a|$.

(2) If $\rho$ occurs in $\theta$ with multiplicity one, then $\left\langle\operatorname{tr}\left(\rho_{Z}\right), \operatorname{tr}_{\zeta}(\boldsymbol{\theta})\right\rangle \neq 0$.

Proof. Let $\rho_{i}, 1 \leqslant i \leqslant r$, be the $Z$-stable irreducible components of $\theta$, repeated according to multiplicity. Thus

$$
\theta=\bigoplus_{i=1}^{r} \rho_{i} \oplus \vartheta,
$$

where $\vartheta$ is a sum of $Z$-irrelevant irreducible representations of $T$. It follows that

$$
\boldsymbol{\theta}=\boldsymbol{\vartheta} \oplus \bigoplus_{i=1}^{r} \boldsymbol{\rho}_{i},
$$

for representations $\boldsymbol{\vartheta}, \boldsymbol{\rho}_{i}$ of $Z T$ such that $\boldsymbol{\rho}_{i} \mid T \cong \rho_{i}$ and $\boldsymbol{\vartheta} \mid T \cong \vartheta$. By (A2.1.1), $(\mathrm{A} 2.1 .2)$,

$$
\operatorname{tr}_{\zeta}(\boldsymbol{\theta})=\sum_{i} \alpha_{i} \operatorname{tr}\left(\rho_{i, Z}\right)
$$

for various roots of unity $\alpha_{i}$. The class functions $\operatorname{tr}\left(\rho_{i, Z}\right)$, for distinct $\rho_{i}$, are linearly independent and both assertions follow.

We will not actually use part (1) of the proposition; it is included to add perspective.

We observe that all of the foregoing applies equally to the case where $T$ is a pro $p$-group and $Z$ is a cyclic group of continuous automorphisms of $T$ of finite order relatively prime to $p$. Of course, in this more general context, we have to consider only smooth representations of $T$ and $T^{Z}$.

A2.2. We translate to a broader context. We are given a locally profinite group $G$ and a compact open subgroup $T$ of $G$ which is a pro $p$-group. We are also given a finite cyclic group $Z=\langle\zeta\rangle$ of continuous automorphisms of $G$, which has order relatively prime to $p$ and which stabilizes $T$. The group $T^{Z}$ of $Z$-fixed points in $T$ is then closed in $G$ and is a pro $p$-group. We can form the semi-direct product $Z \ltimes G$, topologized so that the obvious map $G \rightarrow Z \ltimes G$ is a homeomorphism of $G$ with an open subgroup of $Z \ltimes G$.

We prove the main technical result of this appendix. 
Proposition. Let $\rho$ be an irreducible smooth representation of $T$ and $g \in G$. Suppose that

(a) $\rho^{\zeta} \cong \rho$,

(b) $g$ commutes with $\zeta$, and

(c) $\operatorname{dim} \operatorname{Hom}_{T \cap T^{g}}\left(\rho, \rho^{g}\right)=1$.

The element $g$ then intertwines the representation $\rho_{Z}$.

Proof. Let $\tau$ be the unique irreducible representation of $T \cap T^{g}$ occurring in both $\rho \mid T \cap T^{g}$ and $\rho^{g} \mid T \cap T^{g}$. The group $T^{g}$ is normalized by $Z$ and the representations $\rho, \rho^{g}$ are both $Z$-stable, whence it follows that $\tau$ is $Z$-stable.

Let $\rho_{*}=\operatorname{Ind}_{T}^{Z T} \rho=\bigoplus_{\chi} \chi \otimes \tilde{\rho}$, where $\chi$ runs over the characters of $Z$, viewed by inflation as one-dimensional representations of $Z T$. The representations $\chi \otimes \tilde{\rho}$ are pairwise distinct, being distinguished by their determinant characters. Surely $\left(\rho^{g}\right)_{*} \cong\left(\rho_{*}\right)^{g}$. Frobenius Reciprocity, in the guise of [17] 4.1.5, gives

$$
\operatorname{dim} \operatorname{Hom}_{Z\left(T \cap T^{g}\right)}\left(\rho_{*}, \rho_{*}^{g}\right)=|Z|,
$$

since $|Z|$ is the number of $T, T$-double cosets contained in $Z T g Z T$. Consequently:

Lemma. Let $\tilde{\rho}$ be some representation of $Z T$ extending $\rho$. There is a unique representation $\tilde{\rho}_{1}$ of $Z T$, extending $\rho$, such that $g$ intertwines $\tilde{\rho}$ with $\tilde{\rho}_{1}$. Moreover,

$$
\operatorname{dim} \operatorname{Hom}_{Z\left(T \cap T^{g}\right)}\left(\tilde{\rho}, \tilde{\rho}_{1}^{g}\right)=1
$$

That is, there is a unique irreducible representation $\hat{\tau}$ of $Z\left(T \cap T^{g}\right)$ occurring in both $\tilde{\rho} \mid Z\left(T \cap T^{g}\right)$ and $\tilde{\rho}_{1}^{g} \mid Z\left(T \cap T^{g}\right)$. It occurs in both with multiplicity one. On the other hand, $\tilde{\rho} \mid Z\left(T \cap T^{g}\right)$ contains some irreducible representation $\tilde{\tau}$ extending $\tau$. We may choose an extension $\tilde{\rho}_{2}$ of $\rho$ so that $\tilde{\tau}$ occurs in $\tilde{\rho}_{2}^{g} \mid$ $Z\left(T \cap T^{g}\right)$ : thus $\operatorname{Hom}_{Z\left(T \cap T^{g}\right)}\left(\tilde{\rho}, \tilde{\rho}_{2}\right) \neq 0$, implying $\tilde{\rho}_{2}=\tilde{\rho}_{1}$ and $\hat{\tau}=\tilde{\tau}$. Put another way, $\hat{\tau} \mid T \cap T^{g}=\tau$.

We apply A2.1 Proposition (2) to the representation $\tau$ of $T \cap T^{g}$, the rôle of $\boldsymbol{\theta}$ being taken by $\tilde{\rho}_{1}^{g} \mid Z\left(T \cap T^{g}\right)$. We deduce that $\left\langle\tau_{Z}, \operatorname{tr}_{\zeta}\left(\tilde{\rho}_{1}^{g}\right)\right\rangle \neq 0$, where the inner product is that on class functions on the group $T^{Z} \cap\left(T^{Z}\right)^{g}$.

By (A2.1.2), the function $\operatorname{tr}_{\zeta}\left(\tilde{\rho}_{1}^{g}\right)$ is a linear combination of the characters $\operatorname{tr} \varphi_{Z}$, where $\varphi$ ranges over the $Z$-stable irreducible components of $\tilde{\rho}_{1}^{g} \mid T \cap$ 
$T^{g}$. However, the function $\operatorname{tr}_{\zeta}\left(\tilde{\rho}_{1}^{g}\right)$ on $T^{Z} \cap\left(T^{Z}\right)^{g}$ is the restriction of $\operatorname{tr}\left(\rho^{g}\right)_{Z}$, multiplied by a root of unity. Thus $\tau_{Z}$ occurs in $\left(\rho^{g}\right)_{Z} \mid T^{Z} \cap\left(T^{Z}\right)^{g}$ with multiplicity one. Surely $\left(\rho^{g}\right)_{Z}=\left(\rho_{Z}\right)^{g}$, so $g$ intertwines $\rho_{Z}$, as required.

A2.3. We apply A2.2 Proposition to proving the Wide Extension Lemma of 7.5. We return to the notation of that paragraph.

We work in the group $G=\mathrm{GL}_{m}(D)$; the automorphism $\zeta$ is conjugation by the element $\varpi^{\alpha}$ of 7.5. The place of the group $T$ is taken by the group $\mathcal{J}$. It will be convenient to take $\mathcal{J}$ as large as possible. This is achieved as follows. The group $J / J^{1}$ is of the form $\mathrm{GL}_{l}\left(\mathbb{k}_{C}\right)$. The element $\varpi^{\alpha}$ acts here, its group of fixed points being of the form $J_{Z} / J_{Z}^{1} \cong \mathrm{GL}_{r}\left(\mathbb{k}^{\prime}\right)$, for an integer $r$ and a finite extension $\mathbb{k}^{\prime} / \mathbb{k}_{C}$. We may take for $\mathcal{J}$ the inverse image in $J$ of the unipotent radical of a Borel subgroup of $J_{Z} / J_{Z}^{1}$.

The restriction of $\Lambda_{\mathrm{w}}$ to $J$ is a wide extension $\kappa$ of $\eta$. Because of (2.5.1)(4), we may apply A2.2 Proposition to $\kappa$ to show that the representation $\vartheta$ of (7.5.1) is intertwined by every element of the $G_{Z}$-centralizer of $\beta$. The desired result is then given by the following general property of wide extensions, for which we revert to the base field $F$ and the notation of $\S 2$.

Lemma. Let $[\mathfrak{A}, \ell, 0, \beta]$ be a simple stratum in $A=\mathrm{M}_{m}(D)$, and let $B$ denote the $A$-centralizer of $\beta$. Let $X$ be a subgroup of $J=J(\beta, \mathfrak{A})$, containing $J^{1}=$ $J^{1}(\beta, \mathfrak{A})$, such that $X / J^{1}$ is the unipotent radical of the finite reductive group $J / J^{1}$.

Let $\rho$ be an irreducible representation of $X$ such that $\rho \mid J^{1}$ is equivalent to the unique irreducible representation $\eta_{\theta}$ of $J^{1}$ containing a simple character $\theta \in \mathfrak{C}\left(\mathfrak{A}, \beta, \psi_{F}\right)$. Suppose that $\rho$ is intertwined by every element of $B^{\times}$. We then have $\rho \cong \kappa \mid X$, for any wide extension $\kappa$ of $\eta$.

Proof. Let $\kappa$ be some wide extension of $\eta$, and write $\kappa_{X}=\kappa \mid X$. By (2.5.5), the representation $\kappa_{X}$ does not depend on the choice of $\kappa$. Since $\rho\left|J^{1} \cong \eta \cong \kappa_{X}\right| J^{1}$, there is a character $\phi$ of $X$, trivial on $J^{1}$, such that $\rho \cong \phi \otimes \kappa_{X}$. The intertwining property of $\rho$ implies that $\phi$ is intertwined by every element of $B^{\times}$. This implies that $\phi$ is trivial, as required.

Remark. This argument applies in considerably greater generality, but we only need the restricted case treated above. 


\section{REFERENCES}

1. A. Badulescu, Orthogonalité des caractères pour $\mathrm{GL}_{n}$ sur un corps local de caractéristique non nulle, Manuscripta Mathematica 101 (2000), 49-70.

2. _ Correspondance de Jacquet-Langlands en caractéristique non nulle, Ann. Scient. École Norm. Sup. (4) 35 (2002), 695-747.

3. A. Borel, Admissible representations of a semisimple group over a local field with vectors fixed under an Iwahori subgroup, Invent. Math. 35 (1976), 233-259.

4. P. Broussous, Extension du formalisme de Bushnell-Kutzko au cas d'une algèbre à division, Proc. London Math. Soc. (3) 77 (1998), 292-326.

5. P. Broussous and B. Lemaire, Building of $\mathrm{GL}(m, D)$ and centralizers, Transform. Groups 7 (2002), 15-50.

6. C.J. Bushnell and A. Fröhlich, Gauss sums and p-adic division algebras, Lecture Notes in Math., vol. 987, Springer, Berlin-Heidelberg New York, 1983.

7. _ Non-abelian congruence Gauss sums and p-adic simple algebras, Proc. London Math. Soc. (3) 50 (1985), 207-264.

8. C.J. Bushnell and G. Henniart, Local tame lifting for GL(N) I: simple characters, Publ. Math. I.H.É.S. 83 (1996), 105-233.

9. L Local Jacquet-Langlands correspondence and parametric degrees, Manuscripta Math. 114 (2004), 1-7.

10. L__ Local tame lifting for $\mathrm{GL}(n)$ III: explicit base change and Jacquet-Langlands correspondence, J. reine angew. Math. 508 (2005), 39-100.

11. (2005), 685-710.

12. _ The essentially tame local Langlands correspondence, II: totally ramified representations, Compositio Math. 141 (2005), 979-1011.

13. _ The local Langlands Conjecture for GL(2), Grundlehren der mathematischen Wissenschaften, vol. 335, Springer, 2006.

14. - The essentially tame local Langlands correspondence, III: the general case, Proc. London Math. Soc. doi:10.1112/plms/053 (2010).

15. C.J. Bushnell, G. Henniart and P.C. Kutzko, Types and explicit Plancherel formulae for reductive p-adic groups, Clay Math. Inst. Proceedings, to appear.

16. C.J.Bushnell, G. Henniart and B. Lemaire, Caractère et degré formel pour les formes intérieures de $\mathrm{GL}(n)$ sur un corps local de caractéristique non nulle, Manuscripta Mathematica 131 (2010), 11-24.

17. C.J. Bushnell and P.C. Kutzko, The admissible dual of $G L(N)$ via compact open subgroups, Annals of Math. Studies, vol. 129, Princeton University Press, 1993.

18. _ The admissible dual of $S L(N)$ II, Proc. London Math. Soc. (3) 68 (1992), 317-379.

19. _ Smooth representations of p-adic reductive groups; Structure theory via types, Proc. London Math. Soc. (3) 77 (1998), 582-634.

20. L.J. Corwin and R.E. Howe, Computing characters of tamely ramified p-adic division algebras, Pacific J. Math. 73 (1977), 461-477.

21. P. Deligne, D. Kazhdan and M-F. Vignéras, Représentations des algèbres centrales simples p-adiques, Représentations des groupes réductifs sur un corps local, Hermann, Paris, 1984.

22. J. Dixmier, Les $C^{*}$-algèbres et leurs représentations, Gauthiers-Villars, Paris, 1969.

23. G. Glauberman, Correspondences of characters for relatively prime operator groups, Canadian J. Math. 20 (1968), 1465-1488.

24. M. Grabitz, A. Silberger and E.-W. Zink, Level zero types and Hecke algebras for local central simple algebras, J. Number Theory 91 (2001), 92-125. 
25. J.A. Green, The characters of the finite general linear groups, Trans. Amer. Math. Soc. 80 (1955), 402-447.

26. R.E. Howe, Tamely ramified supercuspidal representations of $G L_{n}$, Pacific J. Math. 73 (1977), 437-460.

27. H. Jacquet and R.P. Langlands, Automorphic forms on $G L(2)$, Lecture Notes in Math., vol. 114, Springer Verlag, 1970.

28. H. Koch and E.-W. Zink, Zur Korrespondenz von Darstellungen der Galois-gruppen und der zentralen Divisionsalgebren über lokalen Körpern (der zahme Fall), Math. Nachr. 98 (1980), 83-119.

29. B. Lemaire, Intégrabilité locale des caractères-distributions de $\mathrm{GL}_{n}(F)$, où $F$ est un corps local non-archimédien de caractéristique quelconque, Compositio Math. 100 (1996), 41-75.

30. Intégrales orbitales sur $\mathrm{GL}(n)$ et corps locaux proches, Ann. Institut Fourier 46 (1996), 1027-1056.

31. I.G. Macdonald, Symmetric functions and Hall polynomials (second edition), Oxford University Press, 1995.

32. D. Montgomery and L. Zippin, Topological transformation groups, Wiley, 1955.

33. A. Moy, Local constants and the tame Langlands correspondence, Amer. J. Math. 108 (1986), 863-929.

34. H. Reimann, Representations of tamely ramified p-adic division and matrix algebras, J. Number Theory 38 (1991), 58-105.

35. I. Reiner, Maximal orders, Academic Press, New York, 1975.

36. A. Roche, Parabolic induction and the Bernstein decomposition, Compositio Math. 134 (2002), 113-133.

37. J. Rogawski, Representations of $G L(n)$ and division algebras over a local field, Duke Math. J. 50 (1983), 161-196.

38. V. Sécherre, Représentations lisses de $\mathrm{GL}_{m}(D)$, I: caractères simples, Bull. Soc. Math. France 132 (2004), 327-396.

39. _ Représentations lisses de $\mathrm{GL}_{m}(D)$, II: $\beta$-extensions, Compositio Math. 141 (2005), $1531-1550$.

40. __ Représentations lisses de $\mathrm{GL}_{m}(D)$, III: types simples, Ann Scient. Éc. Norm. Sup. 38 (2005), 951-977.

41. V. Sécherre and S. Stevens, Représentations lisses de $\mathrm{GL}_{m}(D)$, IV: représentations supercuspidales, J. Inst. Math. Jussieu 7 (2008), 527-574.

42. S. Stevens, Intertwining and supercuspidal types for p-adic classical groups, Proc. Lond. Math. Soc. (3) 83 (2001), 120-140.

43. E.-W. Zink, Representation theory of local division algebras, J. reine angew. Math. 428 (1992), 1-44.

44. $\ldots$ More on embeddings of local fields in simple algebras, J. Number Theory 77 (1999), $51-61$.

Colin J. Bushnell

King's College London

Department of Mathematics,

Strand, London WC2R 2LS, UK.

E-mail: colin.bushnell@kcl.ac.uk 
Guy Henniart

Université de Paris-Sud

Laboratoire de Mathématiques d'Orsay,

Orsay Cedex, F-91405;

CNRS, Orsay cedex, F-91405.

E-mail: Guy.Henniart@math.u-psud.fr 